Supporting Information

\title{
The Alcoholic Solvent Influence on ZnO Synthesis: A Joint Experimental and Theoretical Study
}

\author{
Ankica Šarića,*, Ines Despotovićb and Goran Štefanića \\ aRuđer Bošković Institute, Division of Materials Physics, Centre of Excellence for Advanced \\ Materials and Sensing Devices, Bijenička 54, HR-10002 Zagreb, Croatia \\ ${ }^{\mathrm{b}}$ Ruđer Bošković Institute, Division of Physical Chemistry, Bijenička 54, HR-10002 Zagreb, \\ Croatia
}

*Corresponding Author, tel.: +385 14561 111; fax: +385 14680098

E-mail address: Ankica.Saric@irb.hr (A. Šarić) 
Table S1. Formation of the most stable $\mathrm{ZnO}-\mathrm{CH}_{3}\left(\mathrm{CH}_{2}\right)_{\mathrm{n}} \mathrm{OH}$ monomers via selected bonds (a) coordinate $(\mathrm{Zn}-\mathrm{O})^{\mathrm{a}}$; hydrogen $\left(\mathrm{O}-\mathrm{H}^{\cdots} \mathrm{O}\right)^{(\mathrm{b})}$, their dimers ${ }^{(\mathrm{c})}$ and tetramers ${ }^{(\mathrm{d})}$ in different alcohols: $\mathrm{n} \leq 7$ (the same alcohol is used both as reactant and solvent). Standard state (1M) free energies of interaction in solvent at the M05-2X/6-311++G(2df,2pd) + LANL2DZ// M05-2X/6-31+G(d,p) + LANL2DZ level of theory (in kcal $\mathrm{mol}^{-1}$ ).

\begin{tabular}{|c|c|c|c|c|}
\hline \multirow[t]{2}{*}{ Alcohol } & & \multicolumn{3}{|c|}{$\Delta_{\mathrm{r}} G^{*}$ INT } \\
\hline & $\begin{array}{c}{ }^{(\mathrm{a})} \mathrm{ZnO}- \\
\mathrm{CH}_{3}\left(\mathrm{CH}_{2}\right)_{\mathrm{n}} \mathrm{OH}\end{array}$ & $\begin{array}{c}{ }^{(\mathrm{b})} \mathrm{ZnO}- \\
\mathrm{CH}_{3}\left(\mathrm{CH}_{2}\right)_{\mathrm{n}} \mathrm{OH}\end{array}$ & $\begin{array}{c}{ }^{(\mathrm{c})}(\mathrm{ZnO}- \\
\left.\mathrm{CH}_{3}\left(\mathrm{CH}_{2}\right)_{n} \mathrm{OH}\right)_{2}\end{array}$ & $\begin{array}{c}\text { (d) }(\mathrm{ZnO}- \\
\left.\mathrm{CH}_{3}\left(\mathrm{CH}_{2}\right)_{\mathrm{n}} \mathrm{OH}\right)_{4}\end{array}$ \\
\hline Ethanol(n=1) & -42.04 & -11.84 & -12.51 & -5.39 \\
\hline 1-propanol $(\mathrm{n}=2)$ & -42.88 & -11.49 & -13.65 & -5.35 \\
\hline 1-butanol $(n=3)$ & -43.52 & -12.31 & -14.46 & -7.41 \\
\hline 1-pentanol $(\mathrm{n}=4)$ & -44.10 & -10.44 & -15.57 & -8.09 \\
\hline 1-octanol $(\mathrm{n}=7)$ & -46.50 & -9.42 & -19.22 & -7.54 \\
\hline
\end{tabular}


Table S2. Formation of the $(\mathrm{ZnO})_{12}-(\mathrm{ZnO})_{12}{ }^{(\mathrm{a})}$ and $(\mathrm{ZnO})_{36^{-}}(\mathrm{ZnO})_{36}{ }^{(\mathrm{b})}$ associates in different alcohols. Standard state $(1 \mathrm{M})$ free energies of interaction in solvent at the M05-2X/6$311++\mathrm{G}(2 \mathrm{df}, 2 \mathrm{pd})+\mathrm{LANL} 2 \mathrm{DZ} / / \mathrm{M} 05-2 \mathrm{X} / 6-31+\mathrm{G}(\mathrm{d}, \mathrm{p})+$ LANL2DZ level of theory (in kcal mol$\left.{ }^{1}\right)$.

\begin{tabular}{ccc}
\hline Alcohol & \multicolumn{3}{c}{$\boldsymbol{\Delta}_{\mathbf{r}} \boldsymbol{G}^{*}{ }_{\text {INT }}$} \\
\hline Ethanol & $(\mathbf{Z n O})_{\mathbf{1 2}}-(\mathbf{Z n O})_{\mathbf{1 2}}$ & $(\mathbf{Z n O})_{\mathbf{3 6}}-(\mathbf{Z n O})_{\mathbf{3 6}}$ \\
\hline 1-propanol & -61.48 & -74.82 \\
1-butanol & -63.32 & -89.80 \\
1-pentanol & -67.12 & -95.35 \\
1-octanol & -66.10 & -94.07 \\
\hline
\end{tabular}

(a): According to the reaction : $(\mathrm{ZnO})_{12}+(\mathrm{ZnO})_{12} \rightarrow(\mathrm{ZnO})_{12}-(\mathrm{ZnO})_{12}$; (b): According to the reaction: $(\mathrm{ZnO})_{36}+(\mathrm{ZnO})_{36} \rightarrow(\mathrm{ZnO})_{36}-(\mathrm{ZnO})_{36}$ 
Table S3. Formation of the $(\mathrm{ZnO})_{12}$-alcohol and $(\mathrm{ZnO})_{36}$-alcohol species in different alcohols (the same alcohol is used both as reactant and solvent). Standard state (1M) free energies of interaction $\Delta_{\mathrm{r}} G^{*}$ INT computed by using the SMD solvation model at the M05-2X/6-311++G(2df,2pd) + LANL2DZ// M05-2X/6-31+G(d,p) + LANL2DZ level of theory (in kcal mol-1).

\begin{tabular}{cccc}
\hline Alcohol & \multicolumn{3}{c}{$\boldsymbol{\Delta}_{\mathbf{r}} \boldsymbol{G}^{*} \mathbf{I N T}$} \\
\hline Ethanol & $(\mathbf{Z n O})_{\mathbf{1 2}}$-alcohol $^{(\mathbf{a})}$ & $\mathbf{( Z n O}_{\mathbf{1 2}}$-alcohol $^{(\mathbf{b})}$ & $\mathbf{( Z n O}_{\mathbf{3 6}} \mathbf{- a l c o h o l}^{(\mathbf{c})}$ \\
1-propanol & 2.69 & -2.53 & 2.83 \\
1-butanol & 5.89 & -0.76 & 2.49 \\
1-pentanol & 4.06 & -1.69 & -4.95 \\
1-octanol & 5.58 & 0.03 & 0.75 \\
\hline
\end{tabular}

Binding via coordinate $(\mathrm{Zn}-\mathrm{O})$ bond; (b) Binding via hydrogen $(\mathrm{O}-\mathrm{H} \cdots \mathrm{O})$ bond; (c) Binding via both $\mathrm{O}($ alcohol $)-\mathrm{Zn}(\mathrm{ZnO}$ cluster $)$ and $\mathrm{H}(\mathrm{OH}$-alcohol)-O( $\mathrm{ZnO}$ cluster) bonds. 
Table S4. Bond lengths (d), energies $(E)$ and QTAIM properties of the selected bonds in the most stable monomers (via coordinate and hydrogen), dimers and tetramers of the investigated systems.

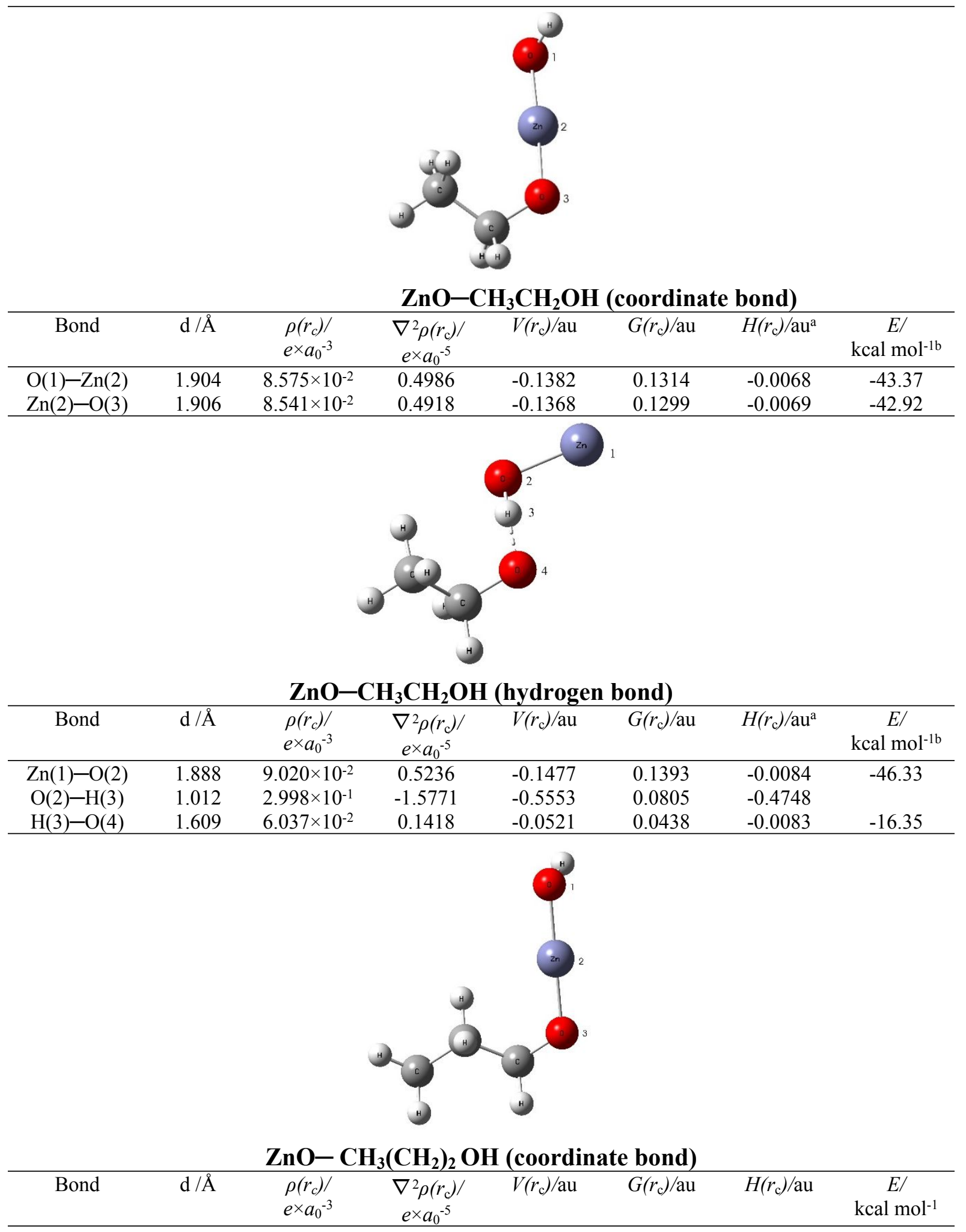




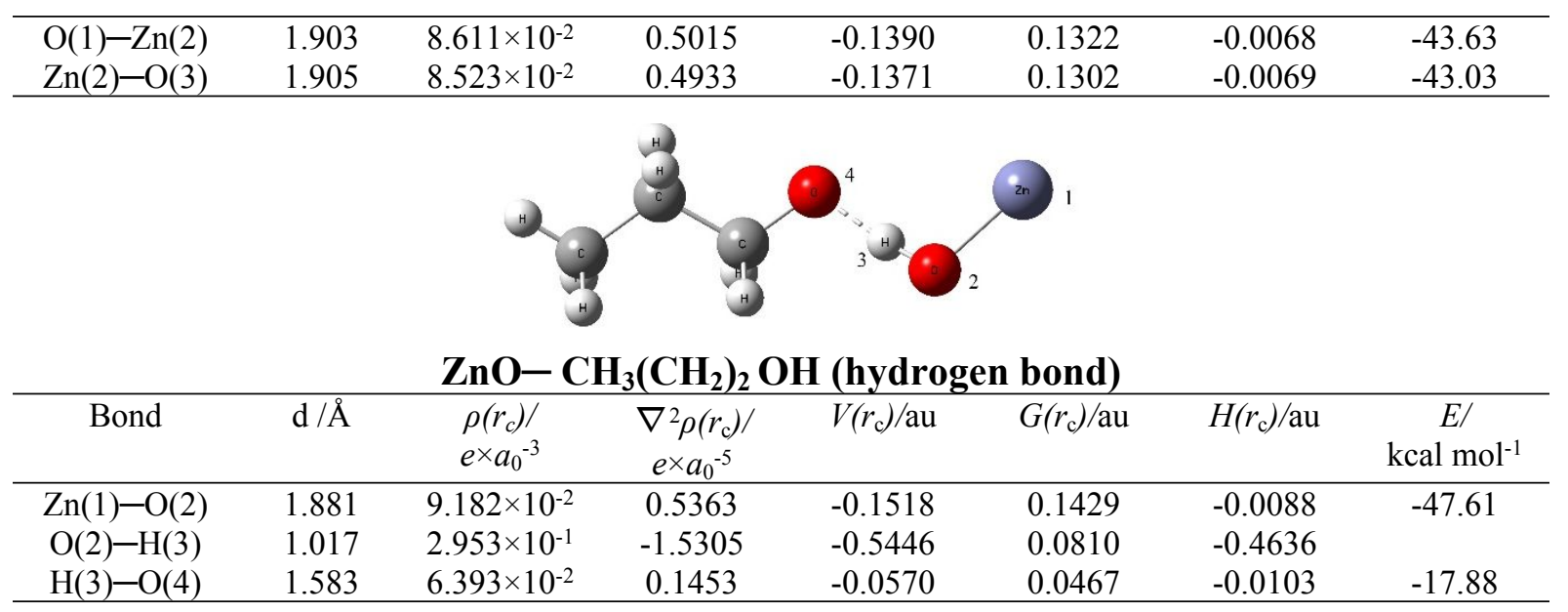

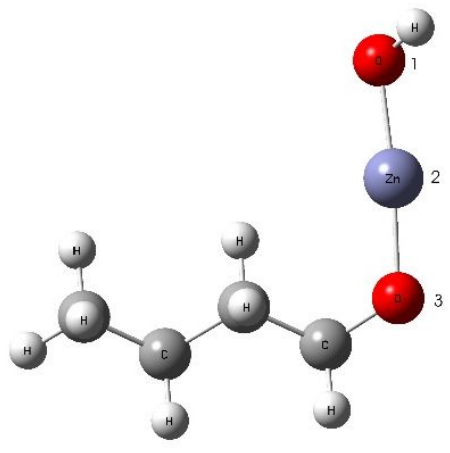

$\mathrm{ZnO}-\mathrm{CH}_{3}\left(\mathrm{CH}_{2}\right)_{3} \mathrm{OH}$ (coordinate bond)

\begin{tabular}{cccccccc}
\hline Bond & $\mathrm{d} / \AA$ & $\begin{array}{c}\rho\left(r_{c}\right) / \\
e^{\times} a_{0}^{-3}\end{array}$ & $\begin{array}{c}\nabla^{2} \rho\left(r_{\mathrm{c}}\right) / \\
e^{\times} a_{0}^{-5}\end{array}$ & $V\left(r_{\mathrm{c}}\right) / \mathrm{au}$ & $G\left(r_{\mathrm{c}}\right) / \mathrm{au}$ & $H\left(r_{\mathrm{c}}\right) / \mathrm{au}$ & $\begin{array}{c}E / \\
\mathrm{kcal} \mathrm{mol}^{-1}\end{array}$ \\
\hline $\mathrm{O}(1)-\mathrm{Zn}(2)$ & 1.897 & $8.723 \times 10^{-2}$ & 0.5133 & -0.1421 & 0.1352 & -0.0069 & -44.59 \\
$\mathrm{Zn}(2)-\mathrm{O}(3)$ & 1.908 & $8.538 \times 10^{-2}$ & 0.4877 & -0.1361 & 0.1290 & -0.0071 & -42.71 \\
\hline
\end{tabular}

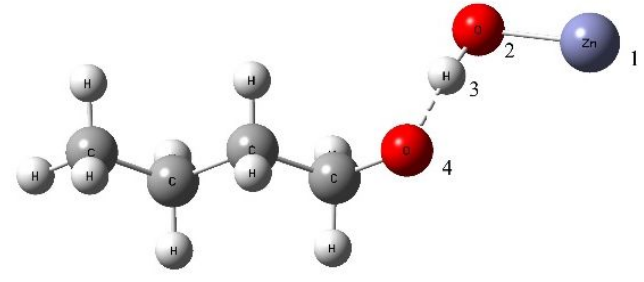

$\mathrm{ZnO}-\mathrm{CH}_{3}\left(\mathrm{CH}_{2}\right)_{3} \mathrm{OH}$ (hydrogen bond)

\begin{tabular}{|c|c|c|c|c|c|c|c|}
\hline Bond & $\bar{d} / \AA$ & $\begin{array}{l}\rho\left(r_{c}\right) / \\
e^{\times} \times a_{0}-3 \\
\end{array}$ & $\begin{array}{l}\nabla^{2} \rho\left(r_{\mathrm{c}}\right) / \\
e^{\times} a_{0}{ }^{-5}\end{array}$ & $V\left(r_{\mathrm{c}}\right) / \mathrm{au}$ & $G\left(r_{\mathrm{c}}\right) / \mathrm{au}$ & $H\left(r_{\mathrm{c}}\right) / \mathrm{au}$ & $\begin{array}{c}E / \\
\text { kcal mol }^{-1}\end{array}$ \\
\hline $\mathrm{Zn}(1)-\mathrm{O}(2)$ & 1.878 & $9.269 \times 10^{-2}$ & 0.5422 & -0.1538 & 0.1447 & -0.0091 & -48.25 \\
\hline $\mathrm{O}(2)-\mathrm{H}(3)$ & 1.020 & $2.921 \times 10^{-1}$ & -1.4938 & -0.5368 & 0.0817 & -0.4551 & \\
\hline $\mathrm{H}(3)-\mathrm{O}(4)$ & 1.568 & $6.655 \times 10^{-2}$ & 0.1457 & -0.0606 & 0.0485 & -0.0121 & -19.01 \\
\hline
\end{tabular}




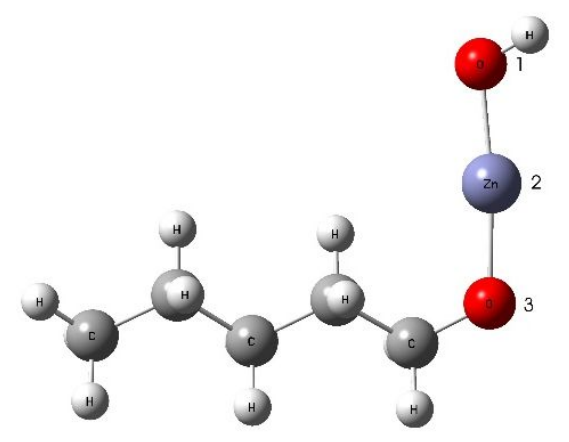

$\mathrm{ZnO}-\mathrm{CH}_{3}\left(\mathrm{CH}_{2}\right)_{4} \mathrm{OH}$ (coordinate bond)

\begin{tabular}{|c|c|c|c|c|c|c|c|}
\hline Bond & $\mathrm{d} / \AA$ & $\begin{array}{l}\rho\left(r_{c}\right) / \\
e^{\times} \times a_{0}{ }^{-3}\end{array}$ & $\begin{array}{l}\nabla^{2} \rho\left(r_{\mathrm{c}}\right) / \\
e^{\times} a_{0}{ }^{-5}\end{array}$ & $V\left(r_{\mathrm{c}}\right) / \mathrm{au}$ & $G\left(r_{\mathrm{c}}\right) / \mathrm{au}$ & $H\left(r_{\mathrm{c}}\right) / \mathrm{au}$ & $\begin{array}{c}E / \\
\text { kcal mol }^{-1}\end{array}$ \\
\hline $\mathrm{O}(1)-\mathrm{Zn}(2)$ & 1.902 & $8.611 \times 10^{-2}$ & 0.5017 & -0.1390 & 0.1322 & -0.0068 & -43.61 \\
\hline $\mathrm{Zn}(2)-\mathrm{O}(3)$ & 1.904 & $8.607 \times 10^{-2}$ & 0.4951 & -0.1380 & 0.1309 & -0.0071 & -43.30 \\
\hline \multicolumn{8}{|c|}{$\mathrm{ZnO}-\mathrm{CH}_{3}\left(\mathrm{CH}_{2}\right)_{4} \mathrm{OH}$ (hydrogen bond) } \\
\hline Bond & $\mathrm{d} / \AA$ & $\begin{array}{l}\rho\left(r_{c}\right) / \\
e \times a_{0}^{-3} \\
\end{array}$ & $\begin{array}{l}\nabla^{2} \rho\left(r_{\mathrm{c}}\right) / \\
e^{\times} a_{0}^{-5}\end{array}$ & $V\left(r_{\mathrm{c}}\right) / \mathrm{au}$ & $G\left(r_{\mathrm{c}}\right) / \mathrm{au}$ & $H\left(r_{\mathrm{c}}\right) / \mathrm{au}$ & $\begin{array}{c}E / \\
\text { kcal mol-1 }\end{array}$ \\
\hline $\mathrm{Zn}(1)-\mathrm{O}(2)$ & 1.875 & $9.305 \times 10^{-2}$ & 0.5464 & -0.1547 & 0.1457 & -0.0091 & -48.54 \\
\hline $\mathrm{O}(2)-\mathrm{H}(3)$ & 1.026 & $2.854 \times 10^{-1}$ & -1.4226 & -0.5211 & 0.0827 & -0.4384 & \\
\hline $\mathrm{H}(3)-\mathrm{O}(4)$ & 1.544 & $7.149 \times 10^{-2}$ & 0.1421 & -0.0673 & 0.0514 & -0.0159 & -21.11 \\
\hline
\end{tabular}

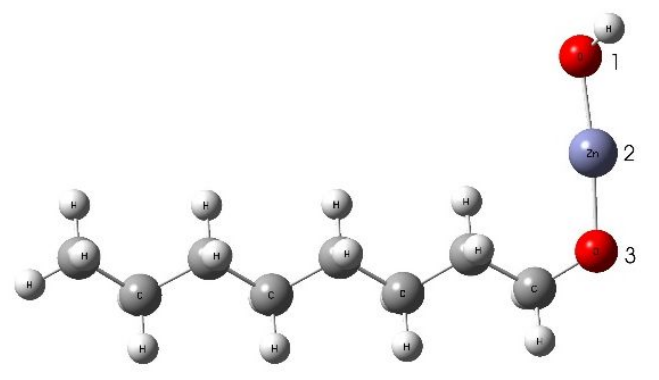

$\mathrm{ZnO}-\mathrm{CH}_{3}\left(\mathrm{CH}_{2}\right)_{7} \mathrm{OH}$ (coordinate bond)

\begin{tabular}{cccccccc}
\hline Bond & $\mathrm{d} / \AA$ & $\begin{array}{c}\rho\left(r_{\mathrm{c}}\right) / \\
e^{\times} a_{0}^{-3}\end{array}$ & $\begin{array}{c}\nabla^{2} \rho\left(r_{\mathrm{c}}\right) / \\
e^{\times} a_{0}^{-5}\end{array}$ & $V\left(r_{\mathrm{c}}\right) / \mathrm{au}$ & $G\left(r_{\mathrm{c}}\right) / \mathrm{au}$ & $H\left(r_{\mathrm{c}}\right) / \mathrm{au}$ & $\begin{array}{c}E / \\
\mathrm{kcal} \mathrm{mol}^{-1}\end{array}$ \\
\hline $\mathrm{O}(1)-\mathrm{Zn}(2)$ & 1.895 & $8.776 \times 10^{-2}$ & 0.5149 & -0.1429 & 0.1358 & -0.0070 & -44.82 \\
$\mathrm{Zn}(2)-\mathrm{O}(3)$ & 1.897 & $8.771 \times 10^{-2}$ & 0.5076 & -0.1417 & 0.1343 & -0.0074 & -44.47 \\
\hline
\end{tabular}




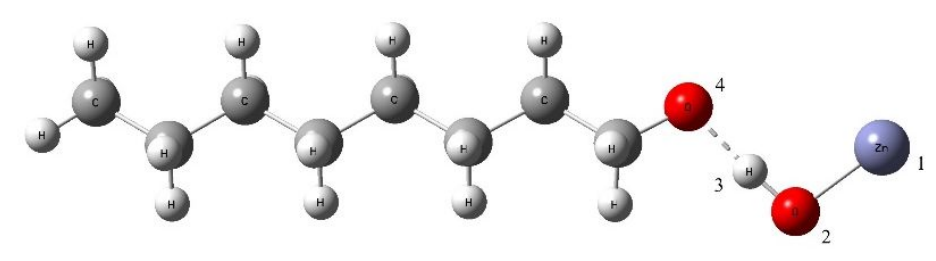

$\mathrm{ZnO}-\mathrm{CH}_{3}\left(\mathrm{CH}_{2}\right)_{7} \mathrm{OH}$ (hydrogen bond)

\begin{tabular}{|c|c|c|c|c|c|c|c|}
\hline Bond & $\mathrm{d} / \AA$ & $\begin{array}{l}\rho\left(r_{c}\right) / \\
e^{\times} \times a_{0}-3 \\
\end{array}$ & $\begin{array}{c}\nabla^{2} \rho\left(r_{\mathrm{c}}\right) / \\
e^{\times} \times a_{0}{ }^{-5}\end{array}$ & $V\left(r_{\mathrm{c}}\right) / \mathrm{au}$ & $G\left(r_{\mathrm{c}}\right) / \mathrm{au}$ & $H\left(r_{\mathrm{c}}\right) / \mathrm{au}$ & $\begin{array}{c}E / \\
\text { kcal mol }^{-1} \\
\end{array}$ \\
\hline $\mathrm{Zn}(1)-\mathrm{O}(2)$ & 1.863 & $9.638 \times 10^{-2}$ & 0.5687 & -0.1628 & 0.1525 & -0.0103 & -51.08 \\
\hline $\mathrm{O}(2)-\mathrm{H}(3)$ & 1.035 & $2.774 \times 10^{-1}$ & -1.3348 & -0.5020 & 0.0841 & -0.4178 & \\
\hline $\mathrm{H}(3)-\mathrm{O}(4)$ & 1.512 & $7.740 \times 10^{-2}$ & 0.1389 & -0.0765 & 0.0556 & -0.0209 & -23.99 \\
\hline
\end{tabular}

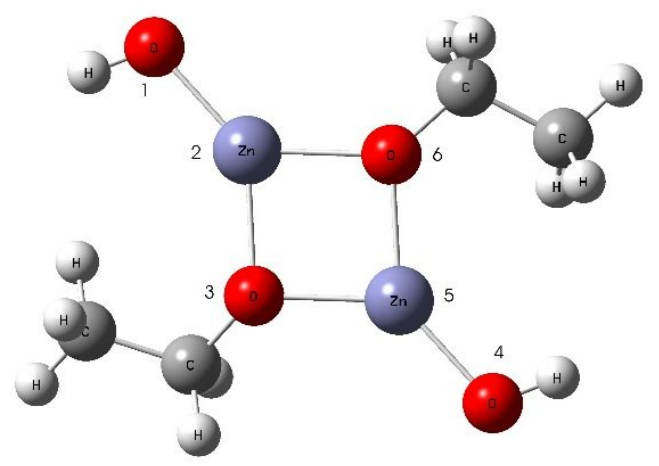

$\left(\mathrm{ZnO}-\mathrm{CH}_{3} \mathrm{CH}_{2} \mathrm{OH}\right)_{2}$

\begin{tabular}{|c|c|c|c|c|c|c|c|}
\hline Bond & $\mathrm{d} / \AA$ & $\begin{array}{l}\rho\left(r_{c}\right) / \\
e \times a_{0}^{-3}\end{array}$ & $\begin{array}{l}\nabla^{2} \rho\left(r_{\mathrm{c}}\right) / \\
e^{\times} a_{0}^{-5}\end{array}$ & $V\left(r_{\mathrm{c}}\right) / \mathrm{au}$ & $G\left(r_{\mathrm{c}}\right) / \mathrm{au}$ & $H\left(r_{\mathrm{c}}\right) / \mathrm{au}$ & $\begin{array}{c}E / \\
\text { kcal mol }{ }^{-1}\end{array}$ \\
\hline $\mathrm{O}(1)-\mathrm{Zn}(2)$ & 1.938 & $7.910 \times 10^{-2}$ & 0.4430 & -0.1237 & 0.1172 & -0.0065 & -38.80 \\
\hline $\mathrm{Zn}(2)-\mathrm{O}(3)$ & 2.020 & $6.317 \times 10^{-2}$ & 0.3233 & -0.0927 & 0.0868 & -0.0059 & -29.09 \\
\hline $\mathrm{Zn}(2)-\mathrm{O}(6)$ & 2.011 & $6.464 \times 10^{-2}$ & 0.3371 & -0.0959 & 0.0901 & -0.0058 & -30.10 \\
\hline $\mathrm{Zn}(3)-\mathrm{O}(5)$ & 2.009 & $6.452 \times 10^{-2}$ & 0.3398 & -0.0962 & 0.0906 & -0.0056 & -30.17 \\
\hline $\mathrm{Zn}(4)-\mathrm{O}(5)$ & 1.939 & $7.895 \times 10^{-2}$ & 0.4418 & -0.1233 & 0.1169 & -0.0064 & -38.69 \\
\hline $\mathrm{Zn}(5)-\mathrm{O}(6)$ & 2.024 & $6.347 \times 10^{-2}$ & 0.3197 & -0.0926 & 0.0862 & -0.0063 & -29.04 \\
\hline
\end{tabular}

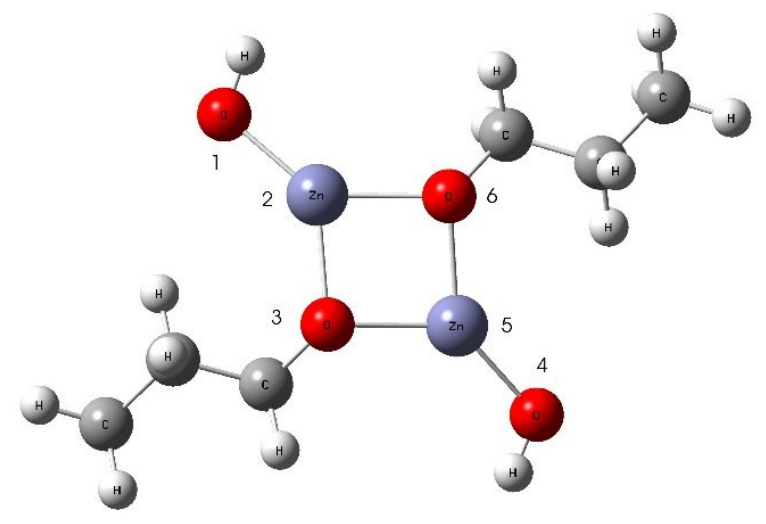

$\left(\mathrm{ZnO}-\mathrm{CH}_{3}\left(\mathrm{CH}_{2}\right)_{2} \mathrm{OH}\right)_{2}$ 


\begin{tabular}{cccccccc}
\hline Bond & $\mathrm{d} / \AA$ & $\begin{array}{c}\rho\left(r_{\mathrm{c}}\right) / \\
e \times a_{0}^{-3}\end{array}$ & $\begin{array}{c}\nabla^{2} \rho\left(r_{\mathrm{c}}\right) / \\
e \times a_{0}^{-5}\end{array}$ & $V\left(r_{\mathrm{c}}\right) / \mathrm{au}$ & $G\left(r_{\mathrm{c}}\right) / \mathrm{au}$ & $H\left(r_{\mathrm{c}}\right) / \mathrm{au}$ & $\begin{array}{c}E / \\
\mathrm{kcal} \mathrm{mol}^{-1}\end{array}$ \\
\hline $\mathrm{O}(1)-\mathrm{Zn}(2)$ & 1.925 & $8.145 \times 10^{-2}$ & 0.4668 & -0.1296 & 0.1232 & -0.0065 & -40.66 \\
$\mathrm{Zn}(2)-\mathrm{O}(3)$ & 2.012 & $6.582 \times 10^{-2}$ & 0.3386 & -0.0972 & 0.0909 & -0.0063 & -30.49 \\
$\mathrm{Zn}(2)-\mathrm{O}(6)$ & 2.027 & $6.311 \times 10^{-2}$ & 0.3186 & -0.0923 & 0.0860 & -0.0060 & -28.96 \\
$\mathrm{Zn}(3)-\mathrm{O}(5)$ & 2.038 & $6.186 \times 10^{-2}$ & 0.3062 & -0.0897 & 0.0831 & -0.0066 & -28.15 \\
$\mathrm{Zn}(4)-\mathrm{O}(5)$ & 1.923 & $8.172 \times 10^{-2}$ & 0.4698 & -0.1303 & 0.1239 & -0.0064 & -40.89 \\
$\mathrm{Zn}(5)-\mathrm{O}(6)$ & 2.011 & $6.575 \times 10^{-2}$ & 0.3388 & -0.0970 & 0.0909 & -0.0062 & -30.44 \\
\hline
\end{tabular}

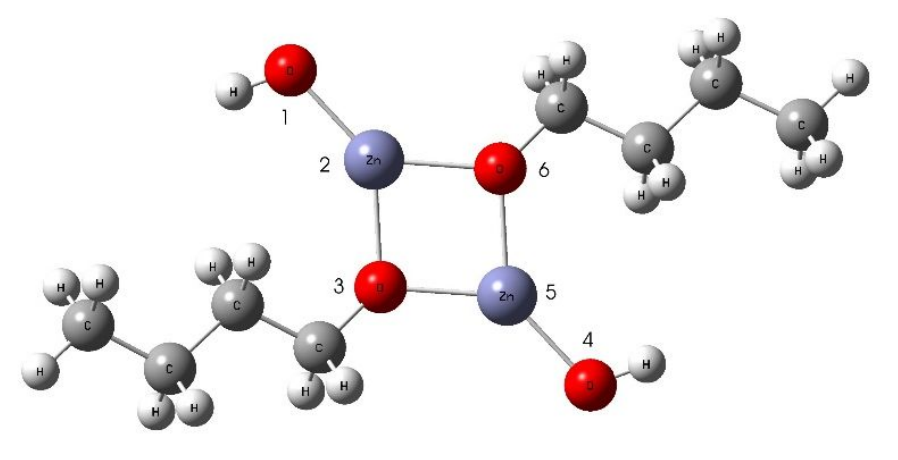

$\left(\mathrm{ZnO}-\mathrm{CH}_{3}\left(\mathrm{CH}_{2}\right)_{3} \mathrm{OH}\right)_{2}$

\begin{tabular}{|c|c|c|c|c|c|c|c|}
\hline Bond & $\mathrm{d} / \AA$ & $\begin{array}{l}\rho\left(r_{c}\right) / \\
e \times a_{0}{ }^{-3}\end{array}$ & $\begin{array}{l}\nabla^{2} \rho\left(r_{c}\right) / \\
e^{\times} a_{0}{ }^{-5}\end{array}$ & $V\left(r_{\mathrm{c}}\right) / \mathrm{au}$ & $G\left(r_{\mathrm{c}}\right) / \mathrm{au}$ & $H\left(r_{\mathrm{c}}\right) / \mathrm{au}$ & $\begin{array}{c}E / \\
\text { kcal mol-1 }\end{array}$ \\
\hline $\mathrm{O}(1)-\mathrm{Zn}(2)$ & 1.931 & $8.032 \times 10^{-2}$ & 0.4546 & -0.1266 & 0.1201 & -0.0065 & -39.72 \\
\hline $\mathrm{Zn}(2)-\mathrm{O}(3)$ & 2.018 & $6.359 \times 10^{-2}$ & 0.3266 & -0.0936 & 0.0876 & -0.0059 & -29.36 \\
\hline $\mathrm{Zn}(2)-\mathrm{O}(6)$ & 1.999 & $6.639 \times 10^{-2}$ & 0.3543 & -0.0997 & 0.0941 & -0.0056 & -31.29 \\
\hline $\mathrm{Zn}(3)-\mathrm{O}(5)$ & 1.997 & $6.666 \times 10^{-2}$ & 0.3571 & -0.1003 & 0.0948 & -0.0055 & -31.47 \\
\hline $\mathrm{Zn}(4)-\mathrm{O}(5)$ & 1.931 & $8.032 \times 10^{-2}$ & 0.4546 & -0.1266 & 0.1201 & -0.0065 & -39.72 \\
\hline $\mathrm{Zn}(5)-\mathrm{O}(6)$ & 2.021 & $6.326 \times 10^{-2}$ & 0.3230 & -0.0928 & 0.0868 & -0.0060 & -29.12 \\
\hline
\end{tabular}

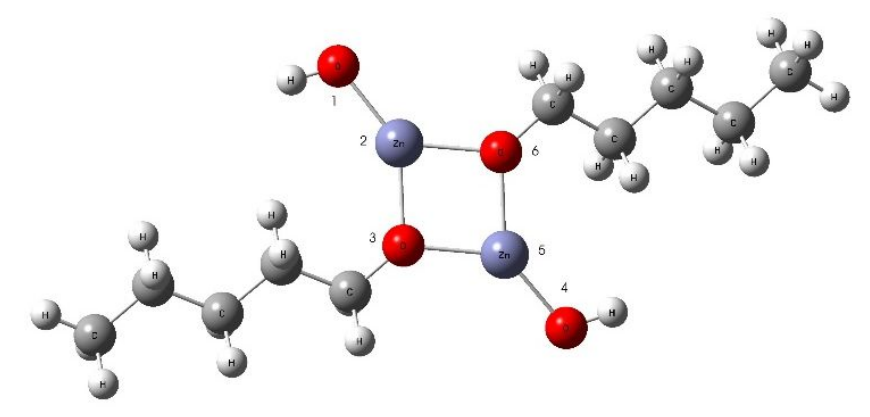

$\left(\mathrm{ZnO}-\mathrm{CH}_{\mathbf{3}}\left(\mathrm{CH}_{2}\right)_{4} \mathrm{OH}\right)_{2}$

\begin{tabular}{cccccccc}
\hline Bond & $\mathrm{d} / \AA$ & $\begin{array}{c}\rho\left(r_{\mathrm{c}}\right) / \\
e^{\times} a_{0}^{-3}\end{array}$ & $\begin{array}{c}\nabla^{2} \rho\left(r_{\mathrm{c}}\right) / \\
e^{\times} a_{0}^{-5}\end{array}$ & $V\left(r_{\mathrm{c}}\right) / \mathrm{au}$ & $G\left(r_{\mathrm{c}}\right) / \mathrm{au}$ & $H\left(r_{\mathrm{c}}\right) / \mathrm{au}$ & $\begin{array}{c}E / \\
\mathrm{kcal} \mathrm{mol}^{-1}\end{array}$ \\
\hline $\mathrm{O}(1)-\mathrm{Zn}(2)$ & 1.928 & $8.092 \times 10^{-2}$ & 0.4608 & -0.1281 & 0.1217 & -0.0065 & -40.20 \\
$\mathrm{Zn}(2)-\mathrm{O}(3)$ & 2.003 & $6.617 \times 10^{-2}$ & 0.3474 & -0.0985 & 0.0927 & -0.0058 & -30.90 \\
$\mathrm{Zn}(2)-\mathrm{O}(6)$ & 2.004 & $6.509 \times 10^{-2}$ & 0.3470 & -0.0976 & 0.0922 & -0.0054 & -30.62 \\
$\mathrm{Zn}(3)-\mathrm{O}(5)$ & 2.007 & $6.517 \times 10^{-2}$ & 0.3442 & -0.0973 & 0.0917 & -0.0056 & -30.53 \\
$\mathrm{Zn}(4)-\mathrm{O}(5)$ & 1.916 & $8.307 \times 10^{-2}$ & 0.4823 & -0.1337 & 0.1271 & -0.0066 & -41.95 \\
$\mathrm{Zn}(5)-\mathrm{O}(6)$ & 2.011 & $6.474 \times 10^{-2}$ & 0.3371 & -0.0959 & 0.0901 & -0.0058 & -30.10
\end{tabular}




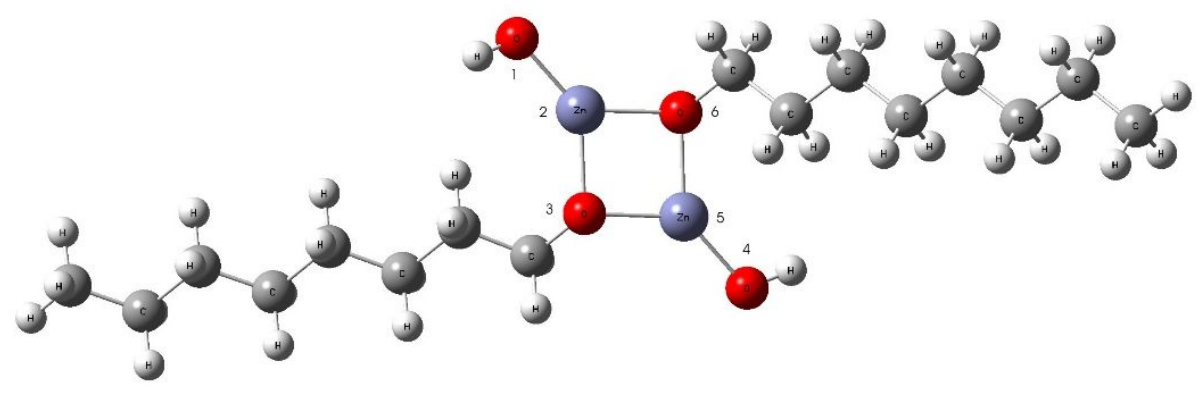

$\left(\mathrm{ZnO}-\mathrm{CH}_{3}\left(\mathrm{CH}_{2}\right)_{7} \mathrm{OH}\right)_{2}$

\begin{tabular}{cccccccc}
\hline Bond & $\mathrm{d} / \AA$ & $\begin{array}{c}\rho\left(r_{\mathrm{c}}\right) / \\
e^{\times} a_{0}^{-3}\end{array}$ & $\begin{array}{c}\nabla^{2} \rho\left(r_{\mathrm{c}}\right) / \\
e^{\times} a_{0}^{-5}\end{array}$ & $V\left(r_{\mathrm{c}}\right) / \mathrm{au}$ & $G\left(r_{\mathrm{c}}\right) / \mathrm{au}$ & $H\left(r_{\mathrm{c}}\right) / \mathrm{au}$ & $\begin{array}{c}E / \\
\mathrm{kcal} \mathrm{mol}^{-1}\end{array}$ \\
\hline $\mathrm{O}(1)-\mathrm{Zn}(2)$ & 1.912 & $8.393 \times 10^{-2}$ & 0.4884 & -0.1355 & 0.1288 & -0.0067 & -42.53 \\
$\mathrm{Zn}(2)-\mathrm{O}(3)$ & 2.004 & $6.654 \times 10^{-2}$ & 0.3483 & -0.0991 & 0.0931 & -0.0060 & -31.09 \\
$\mathrm{Zn}(2)-\mathrm{O}(6)$ & 2.009 & $6.569 \times 10^{-2}$ & 0.3429 & -0.0977 & 0.0917 & -0.0060 & -30.66 \\
$\mathrm{Zn}(3)-\mathrm{O}(5)$ & 2.001 & $6.706 \times 10^{-2}$ & 0.3543 & -0.1005 & 0.0945 & -0.0059 & -31.52 \\
$\mathrm{Zn}(4)-\mathrm{O}(5)$ & 1.913 & $8.375 \times 10^{-2}$ & 0.4869 & -0.1351 & 0.1284 & -0.0067 & -42.38 \\
$\mathrm{Zn}(5)-\mathrm{O}(6)$ & 2.017 & $6.450 \times 10^{-2}$ & 0.3302 & -0.0949 & 0.0887 & -0.0062 & -29.78 \\
\hline
\end{tabular}

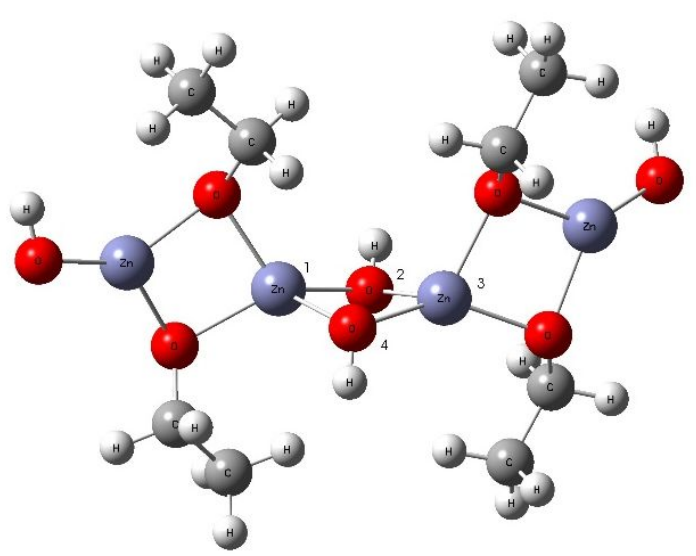

$\left(\mathrm{ZnO}-\mathrm{CH}_{3} \mathrm{CH}_{2} \mathrm{OH}\right)_{4}$

\begin{tabular}{cccccccc}
\hline Bond & $\mathrm{d} / \AA$ & $\begin{array}{c}\rho\left(r_{\mathrm{c}}\right) / \\
e \times a_{0}{ }^{-3}\end{array}$ & $\begin{array}{c}\nabla^{2} \rho\left(r_{\mathrm{c}}\right) / \\
e^{\times} a_{0}^{-5}\end{array}$ & $V\left(r_{\mathrm{c}}\right) / \mathrm{au}$ & $G\left(r_{\mathrm{c}}\right) / \mathrm{au}$ & $H\left(r_{\mathrm{c}}\right) / \mathrm{au}$ & $\begin{array}{c}E / \\
\mathrm{kcal} \mathrm{mol}^{-1}\end{array}$ \\
\hline $\mathrm{Zn}(1)-\mathrm{O}(2)$ & 2.021 & $6.457 \times 10^{-2}$ & 0.3279 & -0.0947 & 0.0884 & -0.0064 & -29.72 \\
$\mathrm{Zn}(1)-\mathrm{O}(4)$ & 2.081 & $5.657 \times 10^{-2}$ & 0.2591 & -0.0796 & 0.0722 & -0.0074 & -24.97 \\
$\mathrm{O}(2)-\mathrm{Zn}(3)$ & 2.076 & $5.716 \times 10^{-2}$ & 0.2647 & -0.0807 & 0.0735 & -0.0073 & -25.33 \\
$\mathrm{Zn}(3)-\mathrm{O}(4)$ & 2.016 & $6.543 \times 10^{-2}$ & 0.3347 & -0.0963 & 0.0899 & -0.0063 & -30.22 \\
\hline
\end{tabular}




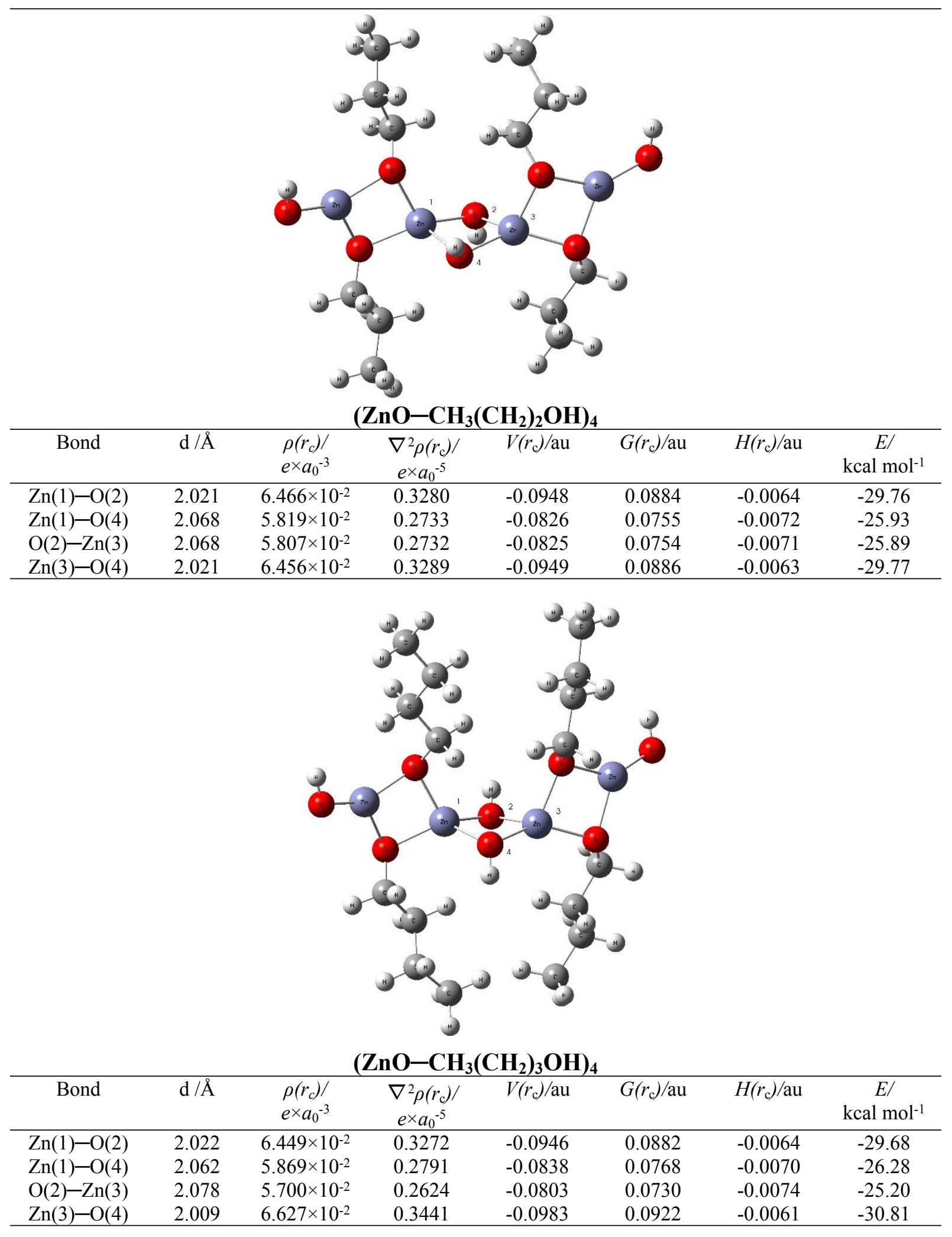




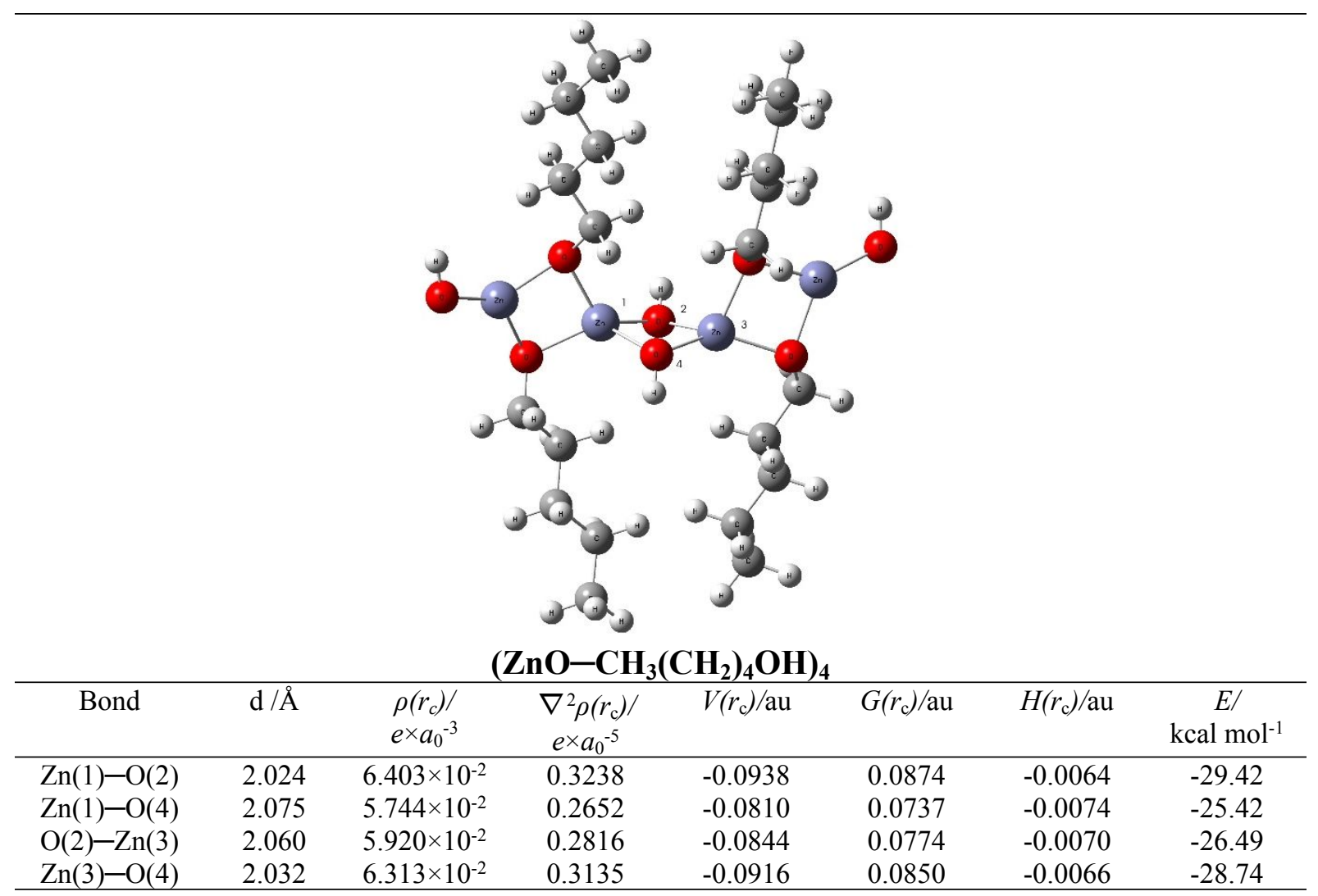




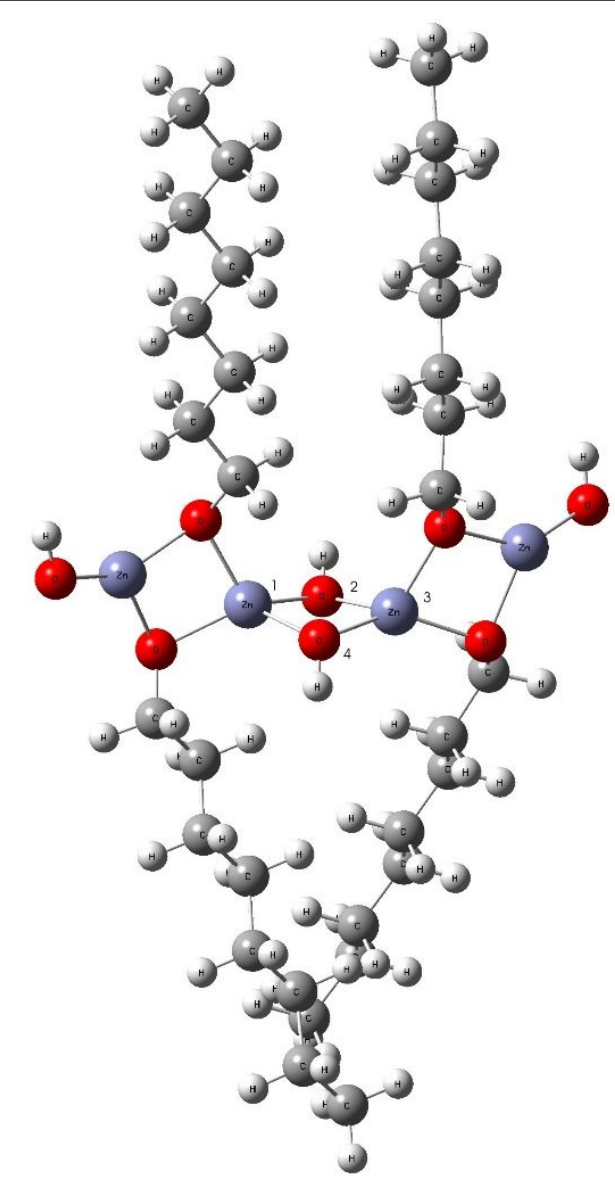

$\left(\mathrm{ZnO}-\mathrm{CH}_{3}\left(\mathrm{CH}_{2}\right)_{7} \mathrm{OH}\right)_{4}$

\begin{tabular}{cccccccc}
\hline Bond & $\mathrm{d} / \AA$ & $\begin{array}{c}\rho\left(r_{\mathrm{c}}\right) / \\
e \times a_{0}{ }^{-3}\end{array}$ & $\begin{array}{c}\nabla^{2} \rho\left(r_{\mathrm{c}}\right) / \\
e \times a_{0}^{-5}\end{array}$ & $V\left(r_{\mathrm{c}}\right) / \mathrm{au}$ & $G\left(r_{\mathrm{c}}\right) / \mathrm{au}$ & $H\left(r_{\mathrm{c}}\right) / \mathrm{au}$ & $\begin{array}{c}E / \\
\mathrm{kcal} \mathrm{mol}^{-1}\end{array}$ \\
\hline $\mathrm{Zn}(1)-\mathrm{O}(2)$ & 2.022 & $6.434 \times 10^{-2}$ & 0.3265 & -0.0943 & 0.0880 & -0.0064 & -29.60 \\
$\mathrm{Zn}(1)-\mathrm{O}(4)$ & 2.050 & $6.027 \times 10^{-2}$ & 0.2934 & -0.0869 & 0.0801 & -0.0068 & -27.26 \\
$\mathrm{O}(2)-\mathrm{Zn}(3)$ & 2.061 & $5.884 \times 10^{-2}$ & 0.2796 & -0.0839 & 0.0769 & -0.0070 & -26.32 \\
$\mathrm{Zn}(3)-\mathrm{O}(4)$ & 2.008 & $6.621 \times 10^{-2}$ & 0.3452 & -0.0984 & 0.0923 & -0.0060 & -30.87 \\
\hline${ }^{\mathrm{a}} H\left(r_{\mathrm{c}}\right)=V\left(r_{\mathrm{c}}\right)+G\left(r_{\mathrm{c}}\right) ;{ }^{b} E=0.5 \times V\left(r_{\mathrm{c}}\right)$ & & & & &
\end{tabular}


Table S5. Bond lengths (d), energies $(E)$ and QTAIM properties of the selected bonds in ( $\mathbf{Z n O})_{12^{-}}$ $(\mathbf{Z n O})_{12}$ and $(\mathbf{Z n O})_{36}-(\mathbf{Z n O})_{36}$ dimers in different solvents.

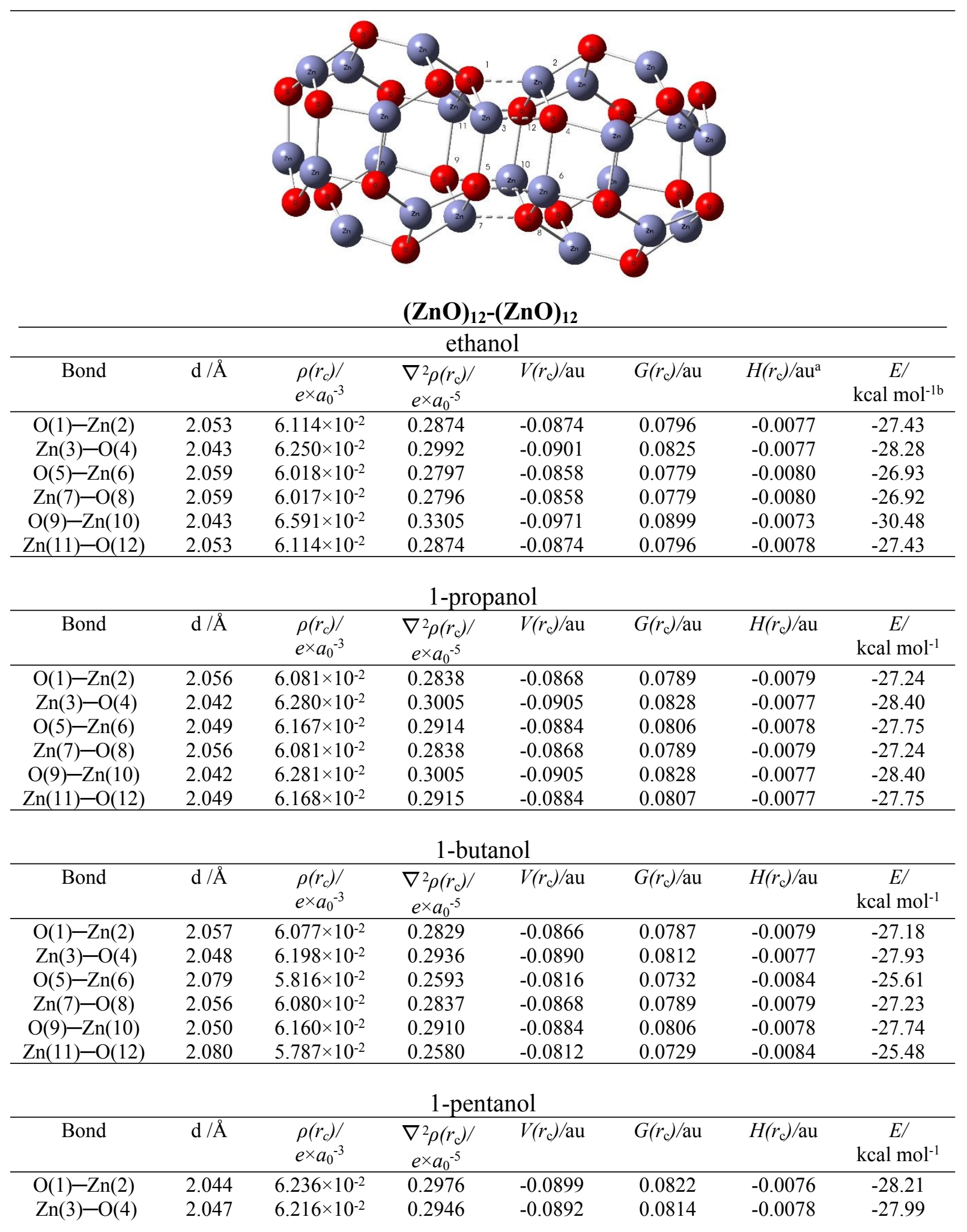




\begin{tabular}{|c|c|c|c|c|c|c|c|}
\hline $\mathrm{O}(5)-\mathrm{Zn}(6)$ & 2.053 & $6.129 \times 10^{-2}$ & 0.2881 & -0.0878 & 0.0799 & -0.0079 & -27.54 \\
\hline $\mathrm{Zn}(7)-\mathrm{O}(8)$ & 2.044 & $6.236 \times 10^{-2}$ & 0.2976 & -0.0899 & 0.0822 & -0.0076 & -28.21 \\
\hline $\mathrm{O}(9)-\mathrm{Zn}(10)$ & 2.047 & $6.216 \times 10^{-2}$ & 0.2946 & -0.0892 & 0.0814 & -0.0078 & -27.99 \\
\hline $\mathrm{Zn}(11)-\mathrm{O}(12)$ & 2.053 & $6.129 \times 10^{-2}$ & 0.2881 & -0.0878 & 0.0799 & -0.0079 & -27.54 \\
\hline \multicolumn{8}{|c|}{ 1-octanol } \\
\hline Bond & $\mathrm{d} / \AA ̊$ & $\begin{array}{l}\rho\left(r_{c}\right) / \\
e \times a_{0}{ }^{-3}\end{array}$ & $\begin{array}{c}\nabla^{2} \rho\left(r_{c}\right) / \\
e^{\times} a_{0}{ }^{-5}\end{array}$ & $V\left(r_{\mathrm{c}}\right) / \mathrm{au}$ & $G\left(r_{\mathrm{c}}\right) / \mathrm{au}$ & $H\left(r_{\mathrm{c}}\right) / \mathrm{au}$ & $\begin{array}{c}E / \\
\text { kcal mol}{ }^{-1}\end{array}$ \\
\hline $\mathrm{O}(1)-\mathrm{Zn}(2)$ & 2.062 & $6.010 \times 10^{-2}$ & 0.2767 & -0.0852 & 0.0772 & -0.0080 & -26.74 \\
\hline $\mathrm{Zn}(3)-\mathrm{O}(4)$ & 2.044 & $6.216 \times 10^{-2}$ & 0.2971 & -0.0896 & 0.0820 & -0.0077 & -28.12 \\
\hline $\mathrm{O}(5)-\mathrm{Zn}(6)$ & 2.042 & $6.241 \times 10^{-2}$ & 0.3001 & -0.0902 & 0.0826 & -0.0076 & -28.31 \\
\hline $\mathrm{Zn}(7)-\mathrm{O}(8)$ & 2.062 & $6.010 \times 10^{-2}$ & 0.2767 & -0.0852 & 0.0772 & -0.0080 & -26.74 \\
\hline $\mathrm{O}(9)-\mathrm{Zn}(10)$ & 2.044 & $6.216 \times 10^{-2}$ & 0.2971 & -0.0896 & 0.0820 & -0.0077 & -28.12 \\
\hline $\mathrm{Zn}(11)-\mathrm{O}(12)$ & 2.042 & $6.241 \times 10^{-2}$ & 0.3001 & -0.0902 & 0.0826 & -0.0076 & -28.31 \\
\hline
\end{tabular}

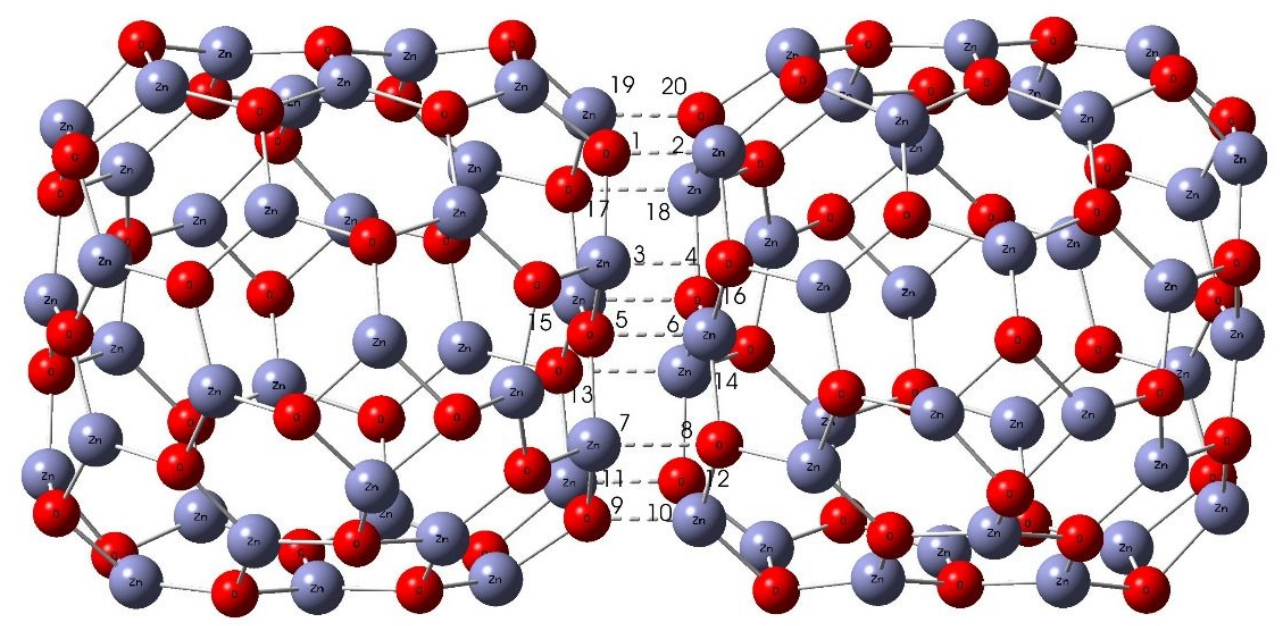

$(\mathrm{ZnO})_{36}-(\mathrm{ZnO})_{36}$

\begin{tabular}{|c|c|c|c|c|c|c|c|}
\hline \multicolumn{8}{|c|}{ ethanol } \\
\hline Bond & $\mathrm{d} / \AA$ & $\begin{array}{l}\rho\left(r_{c}\right) / \\
e \times a_{0}^{-3} \\
\end{array}$ & $\begin{array}{l}\nabla^{2} \rho\left(r_{\mathrm{c}}\right) / \\
e \times a_{0}^{-5} \\
\end{array}$ & $V\left(r_{\mathrm{c}}\right) / \mathrm{au}$ & $G\left(r_{\mathrm{c}}\right) / \mathrm{au}$ & $H\left(r_{\mathrm{c}}\right) / \mathrm{au}$ & $\begin{array}{c}E / \\
\mathrm{kcal} \mathrm{mol}^{-1} \\
\end{array}$ \\
\hline $\mathrm{O}(1)-\mathrm{Zn}(2)$ & 2.161 & $4.901 \times 10^{-2}$ & 0.1884 & -0.0659 & 0.0565 & -0.0094 & -20.68 \\
\hline $\mathrm{Zn}(3)-\mathrm{O}(4)$ & 2.185 & $4.868 \times 10^{-2}$ & 0.1750 & -0.0644 & 0.0541 & -0.0103 & -20.22 \\
\hline $\mathrm{O}(5)-\mathrm{Zn}(6)$ & 2.131 & $5.207 \times 10^{-2}$ & 0.2116 & -0.0711 & 0.0620 & -0.0091 & -22.30 \\
\hline $\mathrm{Zn}(7)-\mathrm{O}(8)$ & 2.042 & $6.292 \times 10^{-2}$ & 0.3018 & -0.0910 & 0.0832 & -0.0078 & -28.56 \\
\hline $\mathrm{O}(9)-\mathrm{Zn}(10)$ & 2.029 & $6.457 \times 10^{-2}$ & 0.3179 & -0.0946 & 0.0870 & -0.0076 & -29.69 \\
\hline $\mathrm{Zn}(11)-\mathrm{O}(12)$ & 2.162 & $4.886 \times 10^{-2}$ & 0.1873 & -0.0657 & 0.0562 & -0.0094 & -20.61 \\
\hline $\mathrm{O}(13)-\mathrm{Zn}(14)$ & 2.184 & $6.357 \times 10^{-2}$ & 0.3094 & -0.0924 & 0.0849 & -0.0075 & -28.99 \\
\hline $\mathrm{Zn}(15)-\mathrm{O}(16)$ & 2.134 & $5.181 \times 10^{-2}$ & 0.2095 & -0.0706 & 0.0615 & -0.0091 & -22.16 \\
\hline $\mathrm{O}(17)-\mathrm{Zn}(18)$ & 2.040 & $6.315 \times 10^{-2}$ & 0.3039 & -0.0915 & 0.0837 & -0.0078 & -28.70 \\
\hline $\mathrm{Zn}(19)-\mathrm{O}(20)$ & 2.030 & $6.452 \times 10^{-2}$ & 0.3173 & -0.0945 & 0.0869 & -0.0076 & -29.65 \\
\hline \multicolumn{8}{|c|}{ 1-propanol } \\
\hline Bond & $\mathrm{d} / \AA \AA$ & $\begin{array}{l}\rho\left(r_{c}\right) / \\
e^{\times} a_{0}^{-3}\end{array}$ & $\begin{array}{l}\nabla^{2} \rho\left(r_{\mathrm{c}}\right) / \\
e^{\times} a_{0}^{-5}\end{array}$ & $V\left(r_{\mathrm{c}}\right) / \mathrm{au}$ & $G\left(r_{\mathrm{c}}\right) / \mathrm{au}$ & $H\left(r_{\mathrm{c}}\right) / \mathrm{au}$ & $\begin{array}{c}E / \\
\mathrm{kcal} \mathrm{mol}^{-1}\end{array}$ \\
\hline
\end{tabular}




\begin{tabular}{cccccccc}
\hline $\mathrm{O}(1)-\mathrm{Zn}(2)$ & 2.138 & $5.130 \times 10^{-2}$ & 0.2066 & -0.0699 & 0.0608 & -0.0092 & -21.92 \\
$\mathrm{Zn}(3)-\mathrm{O}(4)$ & 2.179 & $4.900 \times 10^{-2}$ & 0.1786 & -0.0652 & 0.0549 & -0.0103 & -20.45 \\
$\mathrm{O}(5)-\mathrm{Zn}(6)$ & 2.131 & $5.174 \times 10^{-2}$ & 0.2112 & -0.0707 & 0.0618 & -0.0090 & -22.20 \\
$\mathrm{Zn}(7)-\mathrm{O}(8)$ & 2.030 & $6.435 \times 10^{-2}$ & 0.3159 & -0.0940 & 0.0865 & -0.0075 & -29.50 \\
$\mathrm{O}(9)-\mathrm{Zn}(10)$ & 2.030 & $6.438 \times 10^{-2}$ & 0.3162 & -0.0941 & 0.0866 & -0.0075 & -29.51 \\
$\mathrm{Zn}(11)-\mathrm{O}(12)$ & 2.138 & $5.130 \times 10^{-2}$ & 0.2066 & -0.0699 & 0.0608 & -0.0091 & -21.92 \\
$\mathrm{O}(13)-\mathrm{Zn}(14)$ & 2.179 & $4.899 \times 10^{-2}$ & 0.1786 & -0.0652 & 0.0549 & -0.0103 & -20.45 \\
$\mathrm{Zn}(15)-\mathrm{O}(16)$ & 2.132 & $5.175 \times 10^{-2}$ & 0.2113 & -0.0708 & 0.0618 & -0.0090 & -22.20 \\
$\mathrm{O}(17)-\mathrm{Zn}(18)$ & 2.030 & $6.435 \times 10^{-2}$ & 0.3159 & -0.0940 & 0.0865 & -0.0075 & -29.50 \\
$\mathrm{Zn}(19)-\mathrm{O}(20)$ & 2.030 & $6.438 \times 10^{-2}$ & 0.3161 & -0.0941 & 0.0866 & -0.0075 & -29.51 \\
\hline
\end{tabular}

1-butanol

\begin{tabular}{cccccccc}
\hline Bond & $\mathrm{d} / \AA$ & $\begin{array}{c}\rho\left(r_{\mathrm{c}}\right) / \\
e^{\times} a_{0}^{-3}\end{array}$ & $\begin{array}{c}\nabla^{2} \rho\left(r_{\mathrm{c}}\right) / \\
e^{\times} a_{0}^{-5}\end{array}$ & $V\left(r_{\mathrm{c}}\right) / \mathrm{au}$ & $G\left(r_{\mathrm{c}}\right) / \mathrm{au}$ & $H\left(r_{\mathrm{c}}\right) / \mathrm{au}$ & $\begin{array}{c}E / \\
\mathrm{kcal} \mathrm{mol}^{-1}\end{array}$ \\
\hline $\mathrm{O}(1)-\mathrm{Zn}(2)$ & 2.032 & $6.415 \times 10^{-2}$ & 0.3133 & -0.0936 & 0.0859 & -0.0076 & -29.35 \\
$\mathrm{Zn}(3)-\mathrm{O}(4)$ & 2.143 & $5.071 \times 10^{-2}$ & 0.2016 & -0.0687 & 0.0596 & -0.0092 & -21.58 \\
$\mathrm{O}(5)-\mathrm{Zn}(6)$ & 2.199 & $4.712 \times 10^{-2}$ & 0.1653 & -0.0620 & 0.0516 & -0.0103 & -19.44 \\
$\mathrm{Zn}(7)-\mathrm{O}(8)$ & 2.124 & $5.246 \times 10^{-2}$ & 0.2173 & -0.0721 & 0.0632 & -0.0089 & -22.61 \\
$\mathrm{O}(9)-\mathrm{Zn}(10)$ & 2.037 & $6.346 \times 10^{-2}$ & 0.3077 & -0.0923 & 0.0846 & -0.0077 & -28.96 \\
$\mathrm{Zn}(11)-\mathrm{O}(12)$ & 2.033 & $6.412 \times 10^{-2}$ & 0.3123 & -0.0935 & 0.0857 & -0.0076 & -29.33 \\
$\mathrm{O}(13)-\mathrm{Zn}(14)$ & 2.144 & $5.064 \times 10^{-2}$ & 0.2010 & -0.0686 & 0.0595 & -0.0092 & -21.54 \\
$\mathrm{Zn}(15)-\mathrm{O}(16)$ & 2.199 & $4.717 \times 10^{-2}$ & 0.1656 & -0.0620 & 0.0517 & -0.0103 & -19.46 \\
$\mathrm{O}(17)-\mathrm{Zn}(18)$ & 2.125 & $5.243 \times 10^{-2}$ & 0.2170 & -0.0720 & 0.0631 & -0.0089 & -22.59 \\
$\mathrm{Zn}(19)-\mathrm{O}(20)$ & 2.037 & $6.346 \times 10^{-2}$ & 0.3077 & -0.0923 & 0.0846 & -0.0077 & -28.96 \\
\hline & \multicolumn{7}{c}{}
\end{tabular}

1-pentanol

\begin{tabular}{cccccccc}
\hline Bond & $\mathrm{d} / \AA$ & $\begin{array}{c}\rho\left(r_{\mathrm{c}}\right) / \\
e^{\times} a_{0}^{-3}\end{array}$ & $\begin{array}{c}\nabla^{2} \rho\left(r_{\mathrm{c}}\right) / \\
e^{\times} a_{0}^{-5}\end{array}$ & $V\left(r_{\mathrm{c}}\right) / \mathrm{au}$ & $G\left(r_{\mathrm{c}}\right) / \mathrm{au}$ & $H\left(r_{\mathrm{c}}\right) / \mathrm{au}$ & $\begin{array}{c}E / \\
\mathrm{kcal} \mathrm{mol}^{-1}\end{array}$ \\
\hline $\mathrm{O}(1)-\mathrm{Zn}(2)$ & 2.047 & $6.204 \times 10^{-2}$ & 0.2958 & -0.0895 & 0.0817 & -0.0078 & -28.09 \\
$\mathrm{Zn}(3)-\mathrm{O}(4)$ & 2.153 & $4.960 \times 10^{-2}$ & 0.1937 & -0.0670 & 0.0577 & -0.0093 & -21.01 \\
$\mathrm{O}(5)-\mathrm{Zn}(6)$ & 2.176 & $4.938 \times 10^{-2}$ & 0.1805 & -0.0657 & 0.0554 & -0.0103 & -20.60 \\
$\mathrm{Zn}(7)-\mathrm{O}(8)$ & 2.142 & $5.076 \times 10^{-2}$ & 0.2028 & -0.0690 & 0.0599 & -0.0091 & -21.65 \\
$\mathrm{O}(9)-\mathrm{Zn}(10)$ & 2.028 & $6.478 \times 10^{-2}$ & 0.3196 & -0.0949 & 0.0874 & -0.0075 & -29.77 \\
$\mathrm{Zn}(11)-\mathrm{O}(12)$ & 2.047 & $6.204 \times 10^{-2}$ & 0.2957 & -0.0895 & 0.0817 & -0.0078 & -28.09 \\
$\mathrm{O}(13)-\mathrm{Zn}(14)$ & 2.153 & $4.959 \times 10^{-2}$ & 0.1937 & -0.0670 & 0.0577 & -0.0093 & -21.01 \\
$\mathrm{Zn}(15)-\mathrm{O}(16)$ & 2.176 & $4.938 \times 10^{-2}$ & 0.1805 & -0.0657 & 0.0554 & -0.0103 & -20.60 \\
$\mathrm{O}(17)-\mathrm{Zn}(18)$ & 2.142 & $5.076 \times 10^{-2}$ & 0.2028 & -0.0690 & 0.0598 & -0.0092 & -21.65 \\
$\mathrm{Zn}(19)-\mathrm{O}(20)$ & 2.028 & $6.478 \times 10^{-2}$ & 0.3196 & -0.0949 & 0.0874 & -0.0075 & -29.77 \\
\hline
\end{tabular}

1-octanol

\begin{tabular}{cccccccc}
\hline Bond & $\mathrm{d} / \AA$ & $\begin{array}{c}\rho\left(r_{c}\right) / \\
e \times a_{0}^{-3}\end{array}$ & $\begin{array}{c}\nabla^{2} \rho\left(r_{\mathrm{c}}\right) / \\
e^{\times} a_{0}^{-5}\end{array}$ & $V\left(r_{\mathrm{c}}\right) / \mathrm{au}$ & $G\left(r_{\mathrm{c}}\right) / \mathrm{au}$ & $H\left(r_{\mathrm{c}}\right) / \mathrm{au}$ & $\begin{array}{c}E / \\
\mathrm{kcal} \mathrm{mol}^{-1}\end{array}$ \\
\hline $\mathrm{O}(1)-\mathrm{Zn}(2)$ & 2.143 & $5.074 \times 10^{-2}$ & 0.2019 & -0.0688 & 0.0597 & -0.0092 & -21.60 \\
$\mathrm{Zn}(3)-\mathrm{O}(4)$ & 2.179 & $4.915 \times 10^{-2}$ & 0.1788 & -0.0654 & 0.0550 & -0.0104 & -20.51 \\
$\mathrm{O}(5)-\mathrm{Zn}(6)$ & 2.138 & $5.120 \times 10^{-2}$ & 0.2063 & -0.0697 & 0.0607 & -0.0091 & -21.88 \\
$\mathrm{Zn}(7)-\mathrm{O}(8)$ & 2.042 & $6.281 \times 10^{-2}$ & 0.3016 & -0.0909 & 0.0832 & -0.0077 & -28.52 \\
$\mathrm{O}(9)-\mathrm{Zn}(10)$ & 2.033 & $6.406 \times 10^{-2}$ & 0.3123 & -0.0933 & 0.0857 & -0.0076 & -29.27 \\
$\mathrm{Zn}(11)-\mathrm{O}(12)$ & 2.139 & $5.099 \times 10^{-2}$ & 0.2051 & -0.0694 & 0.0603 & -0.0091 & -21.78 \\
$\mathrm{O}(13)-\mathrm{Zn}(14)$ & 2.178 & $4.921 \times 10^{-2}$ & 0.1793 & -0.0655 & 0.0551 & -0.0103 & -20.54 \\
$\mathrm{Zn}(15)-\mathrm{O}(16)$ & 2.137 & $5.129 \times 10^{-2}$ & 0.2072 & -0.0699 & 0.0609 & -0.0090 & -21.59
\end{tabular}




$\begin{array}{llllllll}\mathrm{O}(17)-\mathrm{Zn}(18) & 2.043 & 6.256 \times 10^{-2} & 0.2997 & -0.0904 & 0.0827 & -0.0077 & -28.38 \\ \mathrm{Zn}(19)-\mathrm{O}(20) & 2.034 & 6.389 \times 10^{-2} & 0.3105 & -0.0929 & 0.0853 & -0.0076 & -29.16\end{array}$

${ }^{\mathrm{a}} H\left(r_{\mathrm{c}}\right)=V\left(r_{\mathrm{c}}\right)+G\left(r_{\mathrm{c}}\right) ;{ }^{b} E=0.5 \times V\left(r_{\mathrm{c}}\right)$

Table S6. Bond lengths (d), energies $(E)$ and QTAIM properties of the selected bonds, coordination $(\mathrm{Zn}-\mathrm{O})$ and hydrogen $(\mathrm{O}-\mathrm{H} \cdots \mathrm{O})$, in the most stable structures of $(\mathrm{ZnO})_{12}-\mathrm{CH}_{3}\left(\mathrm{CH}_{2}\right)_{\mathrm{n}} \mathrm{OH}$, or $(\mathrm{ZnO})_{36}-\mathrm{CH}_{3}\left(\mathrm{CH}_{2}\right)_{\mathrm{n}} \mathrm{OH}, \mathrm{n} \leq 7$.

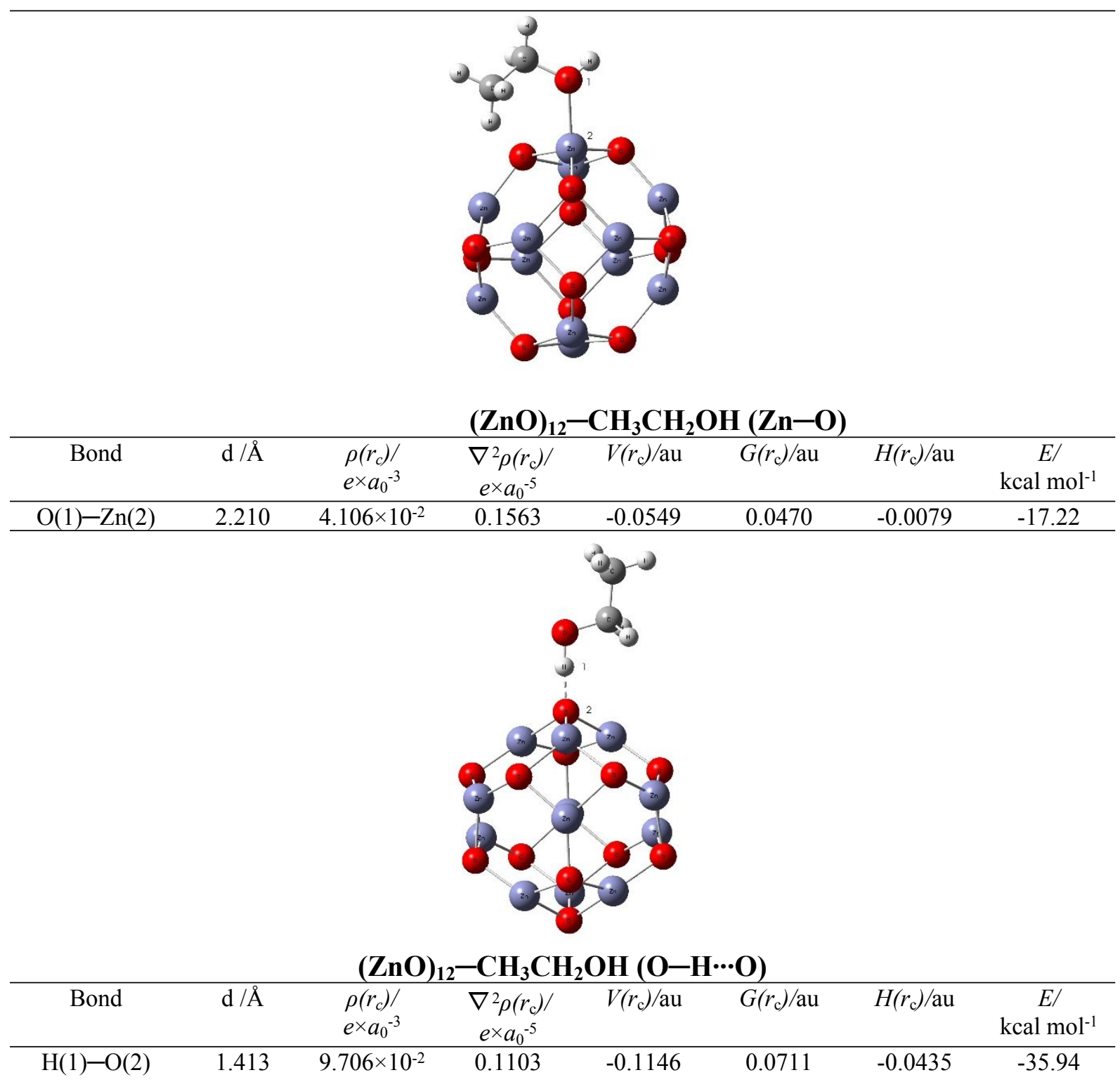




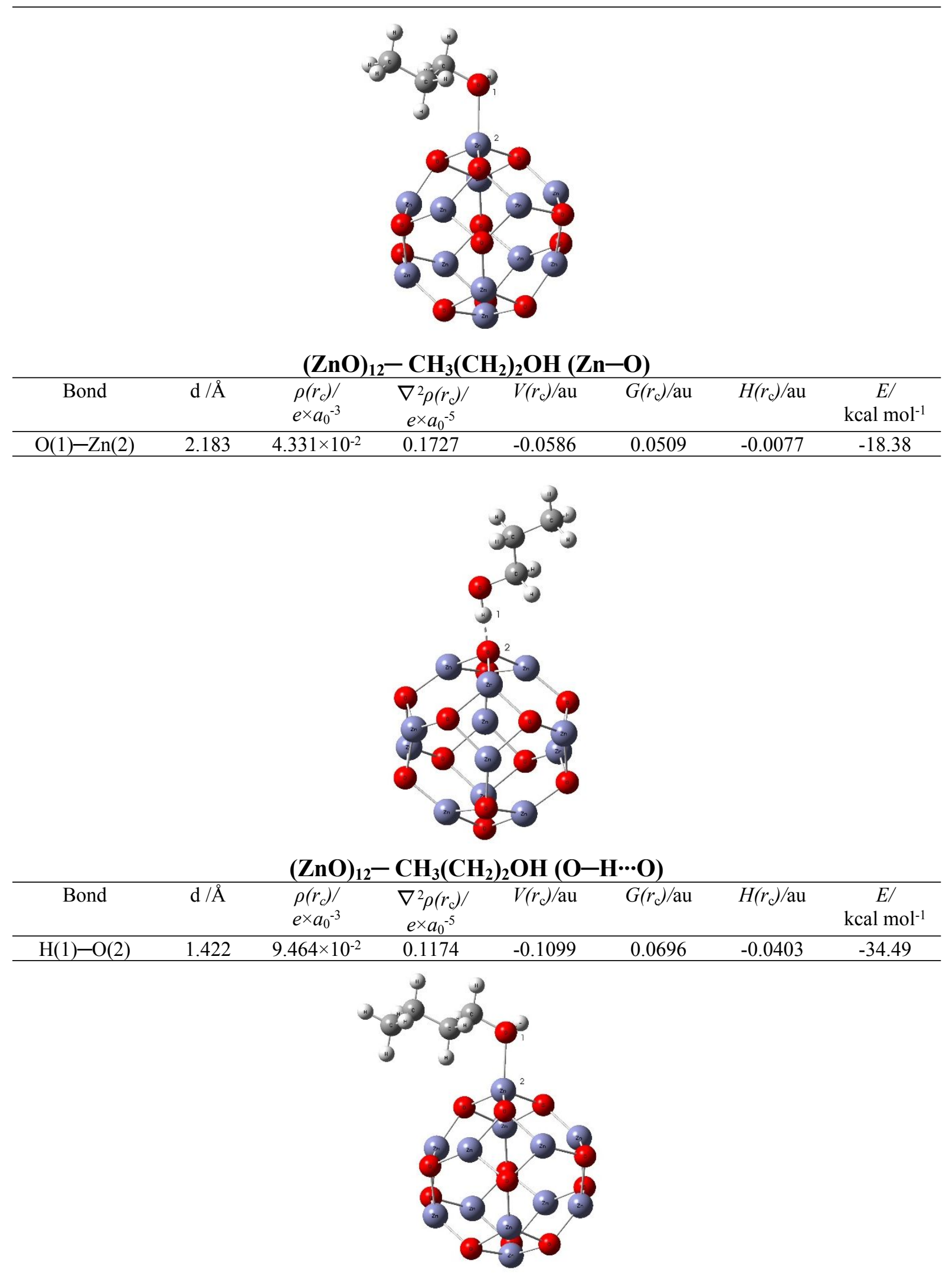




\section{$(\mathrm{ZnO})_{12}-\mathrm{CH}_{\mathbf{3}}\left(\mathrm{CH}_{2}\right)_{3} \mathrm{OH}(\mathrm{Zn}-\mathrm{O})$}

\begin{tabular}{cccccccc}
\hline Bond & $\mathrm{d} / \AA$ & $\begin{array}{c}\rho\left(r_{\mathrm{c}}\right) / \\
e \times a_{0}^{-3}\end{array}$ & $\begin{array}{c}\nabla^{2} \rho\left(r_{\mathrm{c}}\right) / \\
e^{\times} a_{0}{ }^{-5}\end{array}$ & $V\left(r_{\mathrm{c}}\right) / \mathrm{au}$ & $G\left(r_{\mathrm{c}}\right) / \mathrm{au}$ & $H\left(r_{\mathrm{c}}\right) / \mathrm{au}$ & $\begin{array}{c}E / \\
\mathrm{kcal} \mathrm{mol}^{-1}\end{array}$ \\
\hline $\mathrm{O}(1)-\mathrm{Zn}(2)$ & 2.170 & $4.455 \times 10^{-2}$ & 0.1818 & -0.0607 & 0.0531 & -0.0076 & -19.03 \\
\hline
\end{tabular}

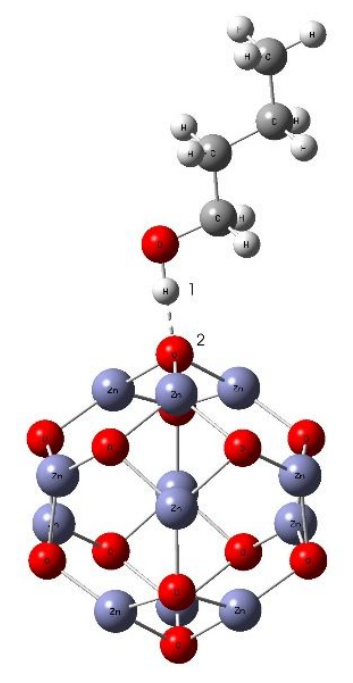

$(\mathrm{ZnO})_{12}-\mathrm{CH}_{\mathbf{3}}\left(\mathrm{CH}_{2}\right)_{\mathbf{3}} \mathrm{OH}(\mathrm{O}-\mathrm{H} \cdots \mathrm{O})$

\begin{tabular}{cccccccc}
\hline Bond & $\mathrm{d} / \AA$ & $\begin{array}{c}\rho\left(r_{\mathrm{c}}\right) / \\
e^{\times} a_{0}{ }^{-3}\end{array}$ & $\begin{array}{c}\nabla^{2} \rho\left(r_{\mathrm{c}}\right) / \\
e^{\times} a_{0}^{-5}\end{array}$ & $V\left(r_{\mathrm{c}}\right) / \mathrm{au}$ & $G\left(r_{\mathrm{c}}\right) / \mathrm{au}$ & $H\left(r_{\mathrm{c}}\right) / \mathrm{au}$ & $\begin{array}{c}E / \\
\mathrm{kcal} \mathrm{mol}^{-1}\end{array}$ \\
\hline $\mathrm{H}(1)-\mathrm{O}(2)$ & 1.422 & $9.370 \times 10^{-2}$ & 0.1203 & -0.1082 & 0.0691 & -0.0390 & -33.93 \\
\hline
\end{tabular}

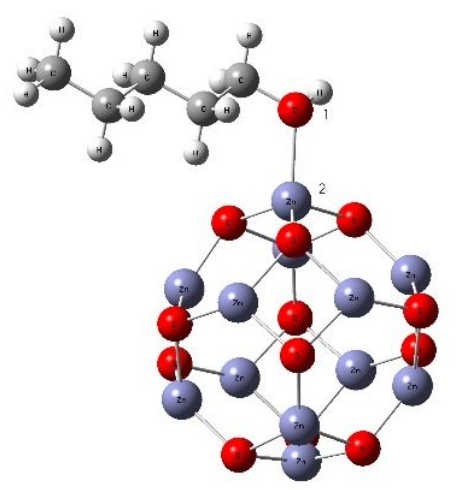

\section{$(\mathrm{ZnO})_{12}-\mathrm{CH}_{\mathbf{3}}\left(\mathrm{CH}_{2}\right)_{\mathbf{4}} \mathrm{OH}(\mathrm{Zn}-\mathrm{O})$}

\begin{tabular}{cccccccc}
\hline Bond & $\mathrm{d} / \AA$ & $\begin{array}{c}\rho\left(r_{\mathrm{c}}\right) / \\
e^{\times} a_{0}^{-3}\end{array}$ & $\begin{array}{c}\nabla^{2} \rho\left(r_{\mathrm{c}}\right) / \\
e^{\times} a_{0}^{-5}\end{array}$ & $V\left(r_{\mathrm{c}}\right) / \mathrm{au}$ & $G\left(r_{\mathrm{c}}\right) / \mathrm{au}$ & $H\left(r_{\mathrm{c}}\right) / \mathrm{au}$ & $\begin{array}{c}E / \\
\mathrm{kcal} \mathrm{mol}^{-1}\end{array}$ \\
\hline $\mathrm{O}(1)-\mathrm{Zn}(2)$ & 2.167 & $4.483 \times 10^{-2}$ & 0.1842 & -0.0676 & 0.0612 & -0.0076 & -19.19
\end{tabular}




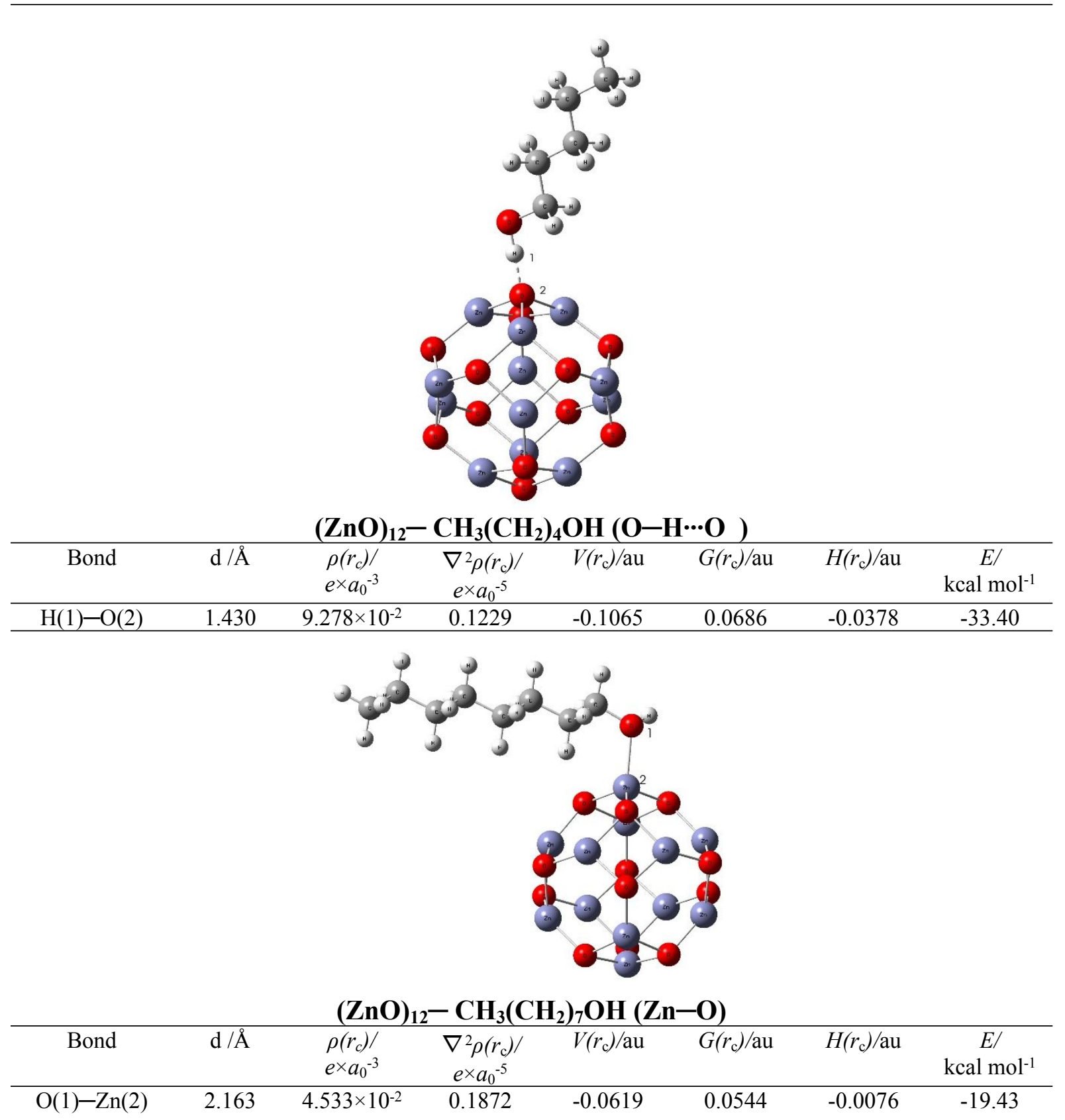




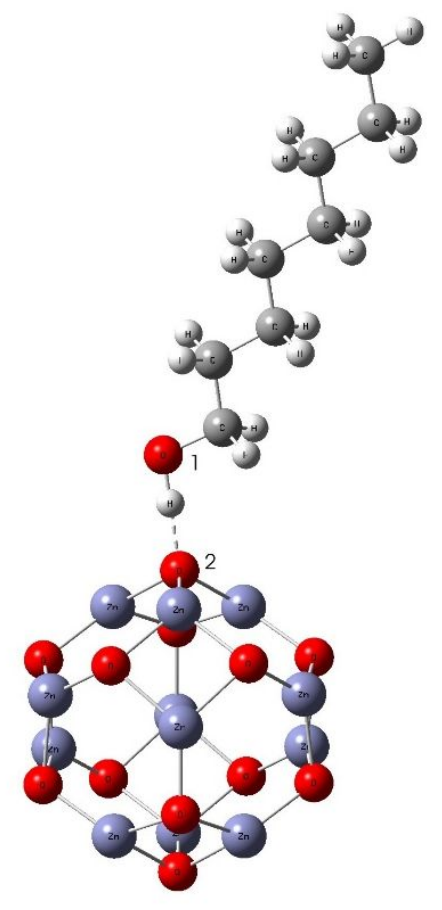

$(\mathrm{ZnO})_{12}-\mathrm{CH}_{\mathbf{3}}\left(\mathrm{CH}_{2}\right)_{\mathbf{7}} \mathrm{OH}(\mathrm{O}-\mathrm{H} \cdots \mathrm{O})$

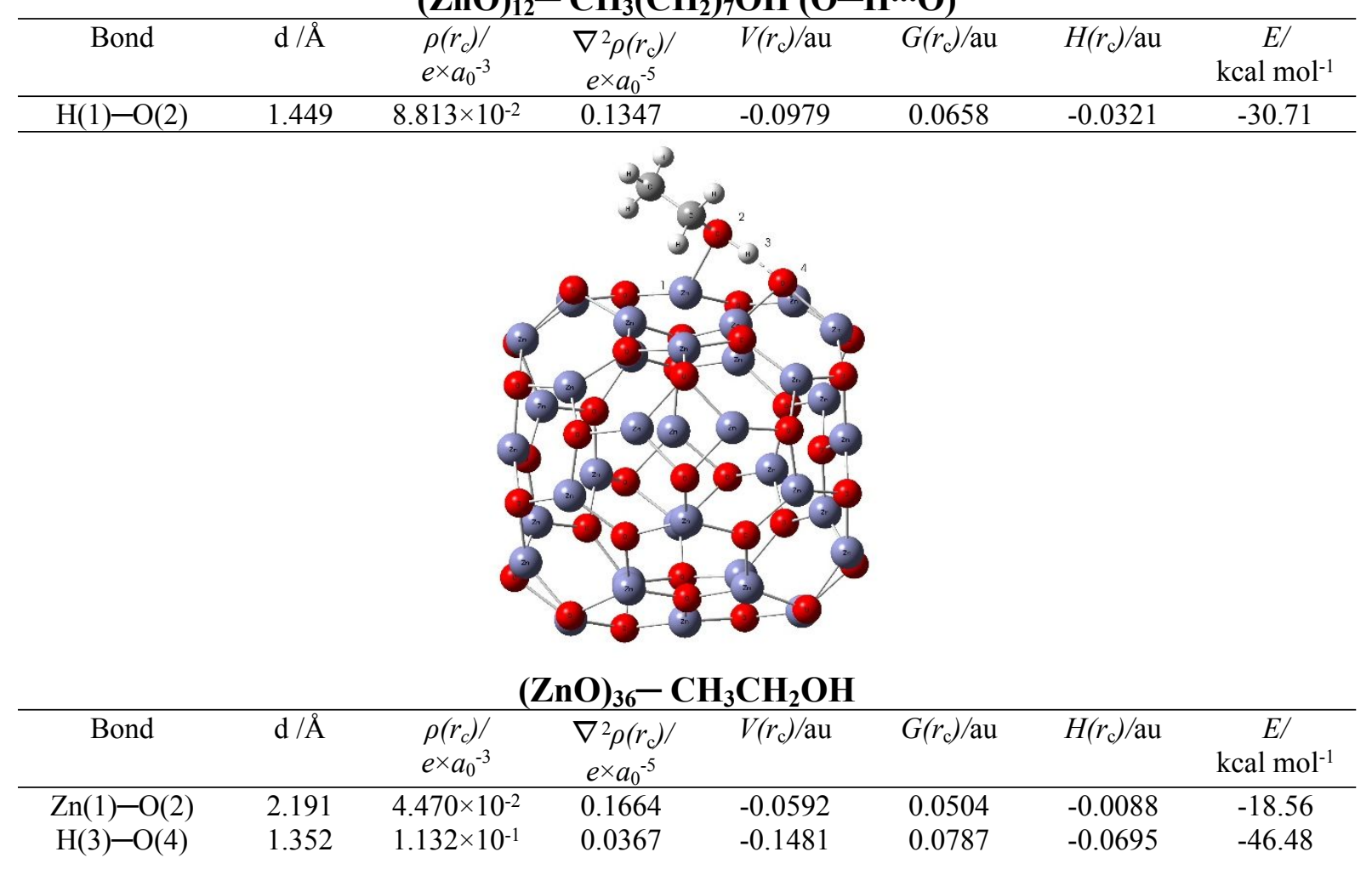




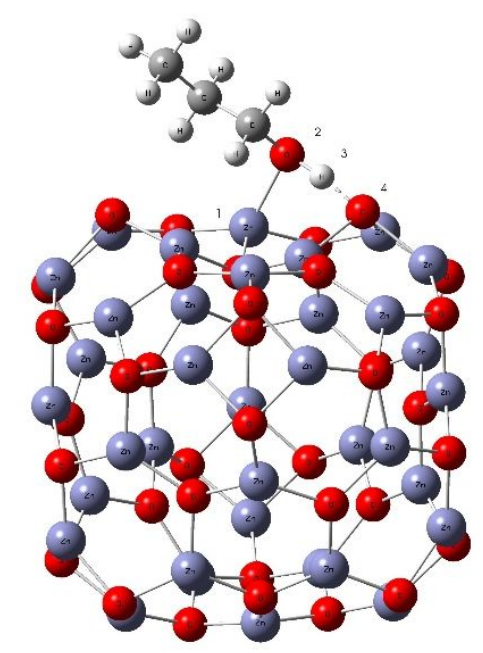

$(\mathrm{ZnO})_{36}-\mathrm{CH}_{3}\left(\mathrm{CH}_{2}\right)_{2} \mathrm{OH}$

\begin{tabular}{cccccccc}
\hline Bond & $\mathrm{d} / \AA$ & $\begin{array}{c}\rho\left(r_{c}\right) / \\
e^{\times} a_{0}^{-3}\end{array}$ & $\begin{array}{c}\nabla^{2} \rho\left(r_{\mathrm{c}}\right) / \\
e^{\times} a_{0}^{-5}\end{array}$ & $V\left(r_{\mathrm{c}}\right) / \mathrm{au}$ & $G\left(r_{\mathrm{c}}\right) / \mathrm{au}$ & $H\left(r_{\mathrm{c}}\right) / \mathrm{au}$ & $\begin{array}{c}E / \\
\mathrm{kcal} \mathrm{mol}^{-1}\end{array}$ \\
\hline $\mathrm{Zn}(1)-\mathrm{O}(2)$ & 2.169 & $4.663 \times 10^{-2}$ & 0.1814 & -0.0625 & 0.0539 & -0.0086 & -19.60 \\
$\mathrm{H}(3)-\mathrm{O}(4)$ & 1.371 & $1.078 \times 10^{-1}$ & 0.0592 & -0.1360 & 0.0754 & -0.0606 & -42.67 \\
\hline
\end{tabular}

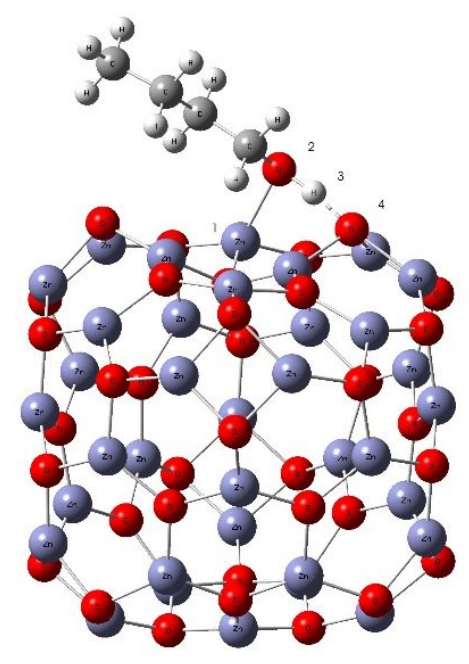

$(\mathrm{ZnO})_{36}-\mathrm{CH}_{3}\left(\mathrm{CH}_{2}\right)_{3} \mathrm{OH}$

\begin{tabular}{cccccccc}
\hline Bond & $\mathrm{d} / \AA$ & $\begin{array}{c}\rho\left(r_{\mathrm{c}}\right) / \\
\mathrm{e} \times a_{0}^{-3}\end{array}$ & $\begin{array}{c}\nabla^{2} \rho\left(r_{\mathrm{c}}\right) / \\
e^{\times} a_{0}^{-5}\end{array}$ & $V\left(r_{\mathrm{c}}\right) / \mathrm{au}$ & $G\left(r_{\mathrm{c}}\right) / \mathrm{au}$ & $H\left(r_{\mathrm{c}}\right) / \mathrm{au}$ & $\begin{array}{c}E / \\
\mathrm{kcal} \mathrm{mol}^{-1}\end{array}$ \\
\hline $\mathrm{Zn}(1)-\mathrm{O}(2)$ & 2.217 & $4.225 \times 10^{-2}$ & 0.1505 & -0.0553 & 0.0464 & -0.0088 & -17.34 \\
$\mathrm{H}(3)-\mathrm{O}(4)$ & 1.374 & $1.071 \times 10^{-1}$ & 0.0695 & -0.1349 & 0.0761 & -0.0587 & -42.32
\end{tabular}




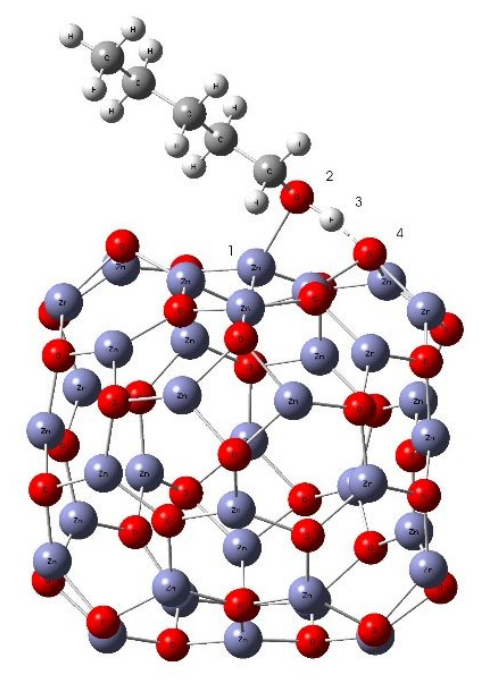

\section{$(\mathrm{ZnO})_{36}-\mathrm{CH}_{3}\left(\mathrm{CH}_{2}\right)_{4} \mathrm{OH}$}

\begin{tabular}{cccccccc}
\hline Bond & $\mathrm{d} / \AA$ & $\begin{array}{c}\rho\left(r_{\mathrm{c}}\right) / \\
e \times a_{0}^{-3}\end{array}$ & $\begin{array}{c}\nabla^{2} \rho\left(r_{\mathrm{c}}\right) / \\
e^{\times} a_{0}^{-5}\end{array}$ & $V\left(r_{\mathrm{c}}\right) / \mathrm{au}$ & $G\left(r_{\mathrm{c}}\right) / \mathrm{au}$ & $H\left(r_{\mathrm{c}}\right) / \mathrm{au}$ & $\begin{array}{c}E / \\
\mathrm{kcal} \mathrm{mol}^{-1}\end{array}$ \\
\hline $\mathrm{Zn}(1)-\mathrm{O}(2)$ & 2.180 & $4.563 \times 10^{-2}$ & 0.1742 & -0.0608 & 0.0522 & -0.0086 & -19.08 \\
$\mathrm{H}(3)-\mathrm{O}(4)$ & 1.366 & $1.092 \times 10^{-1}$ & 0.0591 & -0.1393 & 0.0770 & -0.0623 & -43.71 \\
\hline
\end{tabular}

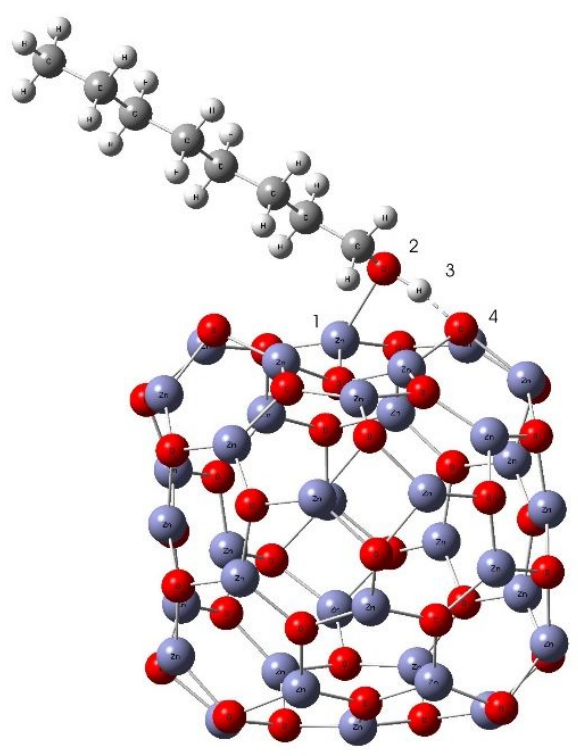

\section{$(\mathrm{ZnO})_{36}-\mathrm{CH}_{3}\left(\mathrm{CH}_{2}\right)_{7} \mathrm{OH}$}

\begin{tabular}{cccccccc}
\hline Bond & $\mathrm{d} / \AA$ & $\begin{array}{c}\rho\left(r_{\mathrm{c}}\right) / \\
e^{\times} a_{0}{ }^{-3}\end{array}$ & $\begin{array}{c}\nabla^{2} \rho\left(r_{\mathrm{c}}\right) / \\
e^{\times} a_{0}^{-5}\end{array}$ & $V\left(r_{\mathrm{c}}\right) / \mathrm{au}$ & $G\left(r_{\mathrm{c}}\right) / \mathrm{au}$ & $H\left(r_{\mathrm{c}}\right) / \mathrm{au}$ & $\begin{array}{c}E / \\
\mathrm{kcal} \mathrm{mol}^{-1}\end{array}$ \\
\hline $\mathrm{Zn}(1)-\mathrm{O}(2)$ & 2.265 & $3.839 \times 10^{-2}$ & 0.1266 & -0.0491 & 0.0408 & -0.0087 & -15.41 \\
$\mathrm{H}(3)-\mathrm{O}(4)$ & 1.440 & $9.080 \times 10^{-2}$ & 0.1244 & -0.1019 & 0.0665 & -0.0354 & -31.96 \\
\hline${ }^{\mathrm{a}} H\left(r_{\mathrm{c}}\right)=V\left(r_{\mathrm{c}}\right)+G\left(r_{\mathrm{c}}\right) ;{ }^{b} E=0.5 \times V\left(r_{\mathrm{c}}\right)$ & & & &
\end{tabular}


Table S7. Total electronic energy, $E^{\text {Tot }}{ }_{\text {soln }}$, obtained at the SMD/M05-2X/6-311++G(2df,2pd) + LANL2DZ//SMD/M05-2X/6-31+G(d,p) + LANL2DZ level of theory, thermal correction to the

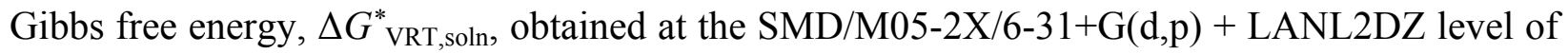
theory, and total free energy, $G_{\mathrm{X}}^{*},\left(G_{\mathrm{X}}^{*}=E^{\mathrm{Tot}}{ }_{\mathrm{soln}}+\Delta G_{\mathrm{VRT}, \text { soln }}^{*}\right)$ in different solvents ${ }^{\mathrm{a}}$ of the investigated species (all energies in hartree).

\begin{tabular}{|c|c|c|c|}
\hline species & $E_{\text {soln }}^{\text {Tot }}$ & $\Delta G_{\text {VRT,soln }}^{*}$ & $G_{\mathrm{X}}^{*}$ \\
\hline $\mathrm{ZnO}$ in ethanol & -140.60794 & -0.02072 & -140.62866 \\
\hline $\mathrm{ZnO}$ in 1-propanol & -140.60516 & -0.02071 & -140.62587 \\
\hline $\mathrm{ZnO}$ in 1-butanol & -140.60224 & -0.02070 & -140.62294 \\
\hline $\mathrm{ZnO}$ in 1-pentanol & -140.59960 & -0.02069 & -140.62029 \\
\hline $\mathrm{ZnO}$ in 1-octanol & -140.58887 & -0.02064 & -140.60952 \\
\hline $\mathrm{CH}_{3} \mathrm{CH}_{2} \mathrm{OH}$ & -155.08227 & 0.05551 & -155.02676 \\
\hline $\mathrm{CH}_{3}\left(\mathrm{CH}_{2}\right)_{2} \mathrm{OH}$ & -194.40332 & 0.08227 & -194.32105 \\
\hline $\mathrm{CH}_{3}\left(\mathrm{CH}_{2}\right)_{3} \mathrm{OH}$ & -233.72423 & 0.10850 & -233.61573 \\
\hline $\mathrm{CH}_{3}\left(\mathrm{CH}_{2}\right)_{4} \mathrm{OH}$ & -273.04548 & 0.13525 & -272.91023 \\
\hline $\mathrm{CH}_{3}\left(\mathrm{CH}_{2}\right)_{7} \mathrm{OH}$ & -391.00863 & 0.21473 & -390.79390 \\
\hline $\mathrm{ZnO}-\mathrm{CH}_{3} \mathrm{CH}_{2} \mathrm{OH}$ & -295.77232 & 0.04990 & --295.72242 \\
\hline $\mathrm{ZnO}-\mathrm{CH}_{3}\left(\mathrm{CH}_{2}\right)_{2} \mathrm{OH}$ & -335.09263 & 0.07738 & -335.01525 \\
\hline $\mathrm{ZnO}-\mathrm{CH}_{3}\left(\mathrm{CH}_{2}\right)_{3} \mathrm{OH}$ & -374.41232 & 0.10430 & -374.30802 \\
\hline $\mathrm{ZnO}-\mathrm{CH}_{3}\left(\mathrm{CH}_{2}\right)_{4} \mathrm{OH}$ & -413.73215 & 0.13142 & -413.60073 \\
\hline $\mathrm{ZnO}-\mathrm{CH}_{3}\left(\mathrm{CH}_{2}\right)_{7} \mathrm{OH}$ & -531.69051 & 0.21298 & -531.47753 \\
\hline$\left(\mathrm{ZnO}-\mathrm{CH}_{3} \mathrm{CH}_{2} \mathrm{OH}\right)_{2}$ & -591.58397 & 0.12291 & -591.46106 \\
\hline$\left(\mathrm{ZnO}-\mathrm{CH}_{3}\left(\mathrm{CH}_{2}\right)_{2} \mathrm{OH}\right)_{2}$ & -670.22473 & 0.17702 & -670.04771 \\
\hline$\left(\mathrm{ZnO}-\mathrm{CH}_{3}\left(\mathrm{CH}_{2}\right)_{3} \mathrm{OH}\right)_{2}$ & -748.86448 & 0.22922 & -748.63526 \\
\hline$\left(\mathrm{ZnO}-\mathrm{CH}_{3}\left(\mathrm{CH}_{2}\right)_{4} \mathrm{OH}\right)_{2}$ & -827.50535 & 0.28188 & -827.22347 \\
\hline$\left(\mathrm{ZnO}-\mathrm{CH}_{3}\left(\mathrm{CH}_{2}\right)_{7} \mathrm{OH}\right)_{2}$ & -1063.42819 & 0.44460 & -1062.98359 \\
\hline$\left(\mathrm{ZnO}-\mathrm{CH}_{3} \mathrm{CH}_{2} \mathrm{OH}\right)_{4}$ & -1183.20951 & 0.27138 & -1182.93813 \\
\hline$\left(\mathrm{ZnO}-\mathrm{CH}_{3}\left(\mathrm{CH}_{2}\right)_{2} \mathrm{OH}\right)_{4}$ & -1340.49454 & 0.38147 & -1340.11307 \\
\hline$\left(\mathrm{ZnO}-\mathrm{CH}_{3}\left(\mathrm{CH}_{2}\right)_{3} \mathrm{OH}\right)_{4}$ & -1497.77596 & 0.48598 & -1497.28998 \\
\hline$\left(\mathrm{ZnO}-\mathrm{CH}_{3}\left(\mathrm{CH}_{2}\right)_{4} \mathrm{OH}\right)_{4}$ & -1655.06026 & 0.59483 & -1654.46543 \\
\hline$\left(\mathrm{ZnO}-\mathrm{CH}_{3}\left(\mathrm{CH}_{2}\right)_{7} \mathrm{OH}\right)_{4}$ & -2126.90589 & 0.92250 & -2125.98339 \\
\hline$(\mathrm{ZnO})_{12}$ in ethanol & -1688.35388 & -0.02431 & -1688.37819 \\
\hline$(\mathrm{ZnO})_{12}$ in 1-propanol & -1688.34625 & -0.02471 & -1688.37096 \\
\hline
\end{tabular}




\begin{tabular}{|c|c|c|c|}
\hline$(\mathrm{ZnO})_{12}$ in 1-butanol & -1688.33843 & -0.02348 & -1688.36191 \\
\hline$(\mathrm{ZnO})_{12}$ in 1-pentanol & -1688.33164 & -0.02562 & -1688.35726 \\
\hline$(\mathrm{ZnO})_{12}$ in 1-octanol & -1688.30489 & -0.02307 & -1688.32796 \\
\hline $\begin{array}{c}(\mathrm{ZnO})_{12}-(\mathrm{ZnO})_{12} \\
\text { in ethanol }\end{array}$ & -3376.83596 & -0.01839 & -3376.85435 \\
\hline $\begin{array}{c}(\mathrm{ZnO})_{12}-(\mathrm{ZnO})_{12} \\
\text { in 1-propanol }\end{array}$ & -3376.82364 & -0.01919 & -3376.84283 \\
\hline $\begin{array}{c}(\mathrm{ZnO})_{12}-(\mathrm{ZnO})_{12} \\
\text { in 1-butanol }\end{array}$ & -3376.81104 & -0.01974 & -3376.83078 \\
\hline $\begin{array}{c}(\mathrm{ZnO})_{12}-(\mathrm{ZnO})_{12} \\
\text { in 1-pentanol }\end{array}$ & -3376.79754 & -0.02234 & -3376.81988 \\
\hline $\begin{array}{c}(\mathrm{ZnO})_{12}-(\mathrm{ZnO})_{12} \\
\text { in 1-octanol }\end{array}$ & -3376.75361 & -0.01252 & -3376.76613 \\
\hline$(\mathrm{ZnO})_{36}$ in ethanol & -5065.28701 & -0.02771 & -5065.31472 \\
\hline$(\mathrm{ZnO})_{36}$ in 1-propanol & -5065.26825 & -0.02214 & -5065.29039 \\
\hline$(\mathrm{ZnO})_{36}$ in 1-butanol & -5065.24999 & -0.01336 & -5065.26335 \\
\hline$(\mathrm{ZnO})_{36}$ in 1-pentanol & -5065.23313 & -0.01541 & -5065.24854 \\
\hline$(\mathrm{ZnO})_{36}$ in 1-octanol & -5065.16746 & -0.01653 & -5065.18399 \\
\hline $\begin{array}{c}(\mathrm{ZnO})_{36}-(\mathrm{ZnO})_{36} \\
\text { in ethanol }\end{array}$ & -10130.75061 & 0.00191 & -10130.74870 \\
\hline $\begin{array}{c}(\mathrm{ZnO})_{36}-(\mathrm{ZnO})_{36} \\
\text { in 1-propanol }\end{array}$ & -10130.71773 & -0.00616 & -10130.72389 \\
\hline $\begin{array}{c}(\mathrm{ZnO})_{36}-(\mathrm{ZnO})_{36} \\
\text { in 1-butanol }\end{array}$ & -10130.68378 & 0.00514 & -10130.67864 \\
\hline $\begin{array}{c}(\mathrm{ZnO})_{36}-(\mathrm{ZnO})_{36} \\
\text { in 1-pentanol }\end{array}$ & -10130.65281 & 0.00580 & -10130.64701 \\
\hline $\begin{array}{c}(\mathrm{ZnO})_{36}-(\mathrm{ZnO})_{36} \\
\text { in 1-octanol }\end{array}$ & -10130.53154 & 0.00951 & -10130.52203 \\
\hline $\begin{array}{c}(\mathrm{ZnO})_{12}-\mathrm{CH}_{3} \mathrm{CH}_{2} \mathrm{OH} \\
\text { (coord.) }\end{array}$ & -1843.45063 & 0.04997 & -1843.40066 \\
\hline$(\mathrm{ZnO})_{12}-\mathrm{CH}_{3} \mathrm{CH}_{2} \mathrm{OH}$ & -1843.45610 & 0.04712 & -1843.40898 \\
\hline
\end{tabular}

(hydrogen) 


\begin{tabular}{|c|c|c|c|}
\hline $\begin{array}{c}(\mathrm{ZnO})_{12}-\mathrm{CH}_{3}\left(\mathrm{CH}_{2}\right)_{2} \mathrm{OH} \\
\text { (coord.) }\end{array}$ & -1882.76549 & 0.08287 & -1882.68262 \\
\hline $\begin{array}{c}(\mathrm{ZnO})_{12}-\mathrm{CH}_{3}\left(\mathrm{CH}_{2}\right)_{2} \mathrm{OH} \\
\text { (hydrogen) }\end{array}$ & -1882.77052 & 0.07729 & -1882.69323 \\
\hline $\begin{array}{c}(\mathrm{ZnO})_{12}-\mathrm{CH}_{3}\left(\mathrm{CH}_{2}\right)_{3} \mathrm{OH} \\
\text { (coord.) }\end{array}$ & -1922.07934 & 0.10816 & -1921.97118 \\
\hline $\begin{array}{c}(\mathrm{ZnO})_{12}-\mathrm{CH}_{3}\left(\mathrm{CH}_{2}\right)_{3} \mathrm{OH} \\
\text { (hydrogen) }\end{array}$ & -1922.08355 & 0.10321 & -1921.98034 \\
\hline $\begin{array}{c}(\mathrm{ZnO})_{12}-\mathrm{CH}_{3}\left(\mathrm{CH}_{2}\right)_{4} \mathrm{OH} \\
\text { (coord.) }\end{array}$ & -1961.39433 & 0.13573 & -1961.25860 \\
\hline $\begin{array}{c}(\mathrm{ZnO})_{12}-\mathrm{CH}_{3}\left(\mathrm{CH}_{2}\right)_{4} \mathrm{OH} \\
\text { (hydrogen) }\end{array}$ & -1961.39813 & 0.13069 & -1961.26744 \\
\hline $\begin{array}{c}(\mathrm{ZnO})_{12}-\mathrm{CH}_{3}\left(\mathrm{CH}_{2}\right)_{7} \mathrm{OH} \\
\text { (coord.) }\end{array}$ & -2079.33229 & 0.21694 & -2079.11535 \\
\hline $\begin{array}{c}(\mathrm{ZnO})_{12}-\mathrm{CH}_{3}\left(\mathrm{CH}_{2}\right)_{7} \mathrm{OH} \\
\text { (hydrogen) }\end{array}$ & -2079.33440 & 0.21322 & -2079.12118 \\
\hline$(\mathrm{ZnO})_{36}-\mathrm{CH}_{3} \mathrm{CH}_{2} \mathrm{OH}$ & -5220.39612 & 0.05914 & -5220.33698 \\
\hline$(\mathrm{ZnO})_{36}-\mathrm{CH}_{3}\left(\mathrm{CH}_{2}\right)_{2} \mathrm{OH}$ & -5259.69914 & 0.09167 & -5259.60747 \\
\hline$(\mathrm{ZnO})_{36}-\mathrm{CH}_{3}\left(\mathrm{CH}_{2}\right)_{3} \mathrm{OH}$ & -5299.00048 & 0.11351 & -5298.88697 \\
\hline$(\mathrm{ZnO})_{36}-\mathrm{CH}_{3}\left(\mathrm{CH}_{2}\right)_{4} \mathrm{OH}$ & -5338.30704 & 0.14707 & -5338.15997 \\
\hline$(\mathrm{ZnO})_{36}-\mathrm{CH}_{3}\left(\mathrm{CH}_{2}\right)_{7} \mathrm{OH}$ & -5456.20441 & 0.22806 & -5455.97635 \\
\hline
\end{tabular}

a)If not explicitely stated, the solvent used is same as the alcohol molecule contained in the species. 


\section{Cartesian Coordinates}

$\mathrm{ZnO}$ in ethanol

\section{$\mathrm{Zn}$ \\ $\mathrm{O}$}

$\mathrm{ZnO}$ in 1-propanol

$\mathrm{Zn}$

$\mathrm{O}$

$\mathrm{ZnO}$ in 1-butanol

$\mathrm{Zn}$

$\mathrm{O}$

$\mathrm{ZnO}$ in 1-pentanol

$\mathrm{Zn}$

$\mathrm{O}$

$\mathrm{ZnO}$ - in 1-octanol

0.000000

0.000000

0.000000

0.000000

0.000000

1.839465

0.000000

0.000000

0.000000

0.000000

0.000000

1.836867

0.000000

0.000000

0.000000

0.000000

0.000000

1.834188

0.000000

0.000000

0.000000

0.000000

0.000000

1.831765

0.000000

0.000000

0.000000

0.000000
1.822283

\section{$\mathrm{CH}_{3} \mathrm{CH}_{2} \mathrm{OH}$}

$\mathrm{C}$
$\mathrm{C}$
$\mathrm{O}$
$\mathrm{H}$
$\mathrm{H}$
$\mathrm{H}$
$\mathrm{H}$
$\mathrm{H}$

1.396599

0.000000

1.894346

$-0.034711$

0.000000

1.405701

$-0.011065$

0.000000

$-0.029568$

1.420136

0.000000

2.986163

1.922888

$-0.887670$

1.536638

1.922888

0.887670

1.536638

$-0.918658$

0.000000

$-0.354670$

$-0.562727$

$-0.562727$

$-0.887704$

1.765209

0.887704

1.765209 
$\mathrm{CH}_{3}\left(\mathrm{CH}_{2}\right)_{2} \mathrm{OH}$

$\begin{array}{lrrr}\mathrm{C} & 2.773888 & -0.006982 & 3.499394 \\ \mathrm{C} & 1.352306 & 0.002119 & 2.941096 \\ \mathrm{C} & 1.356525 & -0.005417 & 1.425508 \\ \mathrm{O} & -0.002182 & 0.013077 & 0.967461 \\ \mathrm{H} & -0.004099 & 0.007105 & 0.003508 \\ \mathrm{H} & 1.861589 & -0.903152 & 1.054271 \\ \mathrm{H} & 1.888097 & 0.873311 & 1.045701 \\ \mathrm{H} & 0.801273 & -0.873370 & 3.296979 \\ \mathrm{H} & 0.816671 & 0.890807 & 3.287627 \\ \mathrm{H} & 3.318400 & -0.896495 & 3.171766 \\ \mathrm{H} & 3.331524 & 0.872368 & 3.166465 \\ \mathrm{H} & 2.764054 & -0.003703 & 4.591097\end{array}$

$\mathrm{CH}_{3}\left(\mathrm{CH}_{2}\right)_{3} \mathrm{OH}$

C

C

C

C

$\mathrm{O}$

$\mathrm{H}$

$\mathrm{H}$

$\mathrm{H}$

$\mathrm{H}$

$\mathrm{H}$

$\mathrm{H}$

$\mathrm{H}$

$\mathrm{H}$

$\mathrm{H}$

$\mathrm{H}$
2.792607

2.780803

1.360168

1.351658

$-0.011900$

$-0.023693$

1.851323

1.879922

0.810712

0.827534

3.310400

3.325243

3.813170

2.290310

2.273816
0.000062

$-0.006709$

0.002991

$-0.005743$

0.014106

0.006794

$-0.904345$

0.872051

$-0.872370$

0.893058

$-0.892141$

0.866318

$-0.007699$

0.890305

$-0.877091$
5.009900

3.482462

2.918587

1.402984

0.958705

$-0.005100$

1.026949

1.016806

3.281176

3.270253

3.115825

3.107873

5.399049

5.397895

5.405948

$\mathrm{CH}_{3}\left(\mathrm{CH}_{2}\right)_{4} \mathrm{OH}$

C
C
C
C
C
O

4.211138

$-0.001129$

5.593897

2.789829

$-0.000556$

5.035090

2.767845

$-0.001325$

3.506986

1.350223

0.000039

2.936063

1.353160

$-0.001164$

1.420336

$-0.006665$

0.002188

0.965027 


$\begin{array}{lrrr}\mathrm{H} & -0.010466 & 0.001204 & 0.001203 \\ \mathrm{H} & 1.868014 & -0.891114 & 1.044027 \\ \mathrm{H} & 1.872734 & 0.885487 & 1.042690 \\ \mathrm{H} & 0.805299 & -0.881758 & 3.289308 \\ \mathrm{H} & 0.807744 & 0.883954 & 3.287766 \\ \mathrm{H} & 3.308269 & -0.882167 & 3.140906 \\ \mathrm{H} & 3.310199 & 0.877955 & 3.140000 \\ \mathrm{H} & 2.249503 & -0.879062 & 5.403309 \\ \mathrm{H} & 2.250611 & 0.879022 & 5.402375 \\ \mathrm{H} & 4.760614 & -0.885497 & 5.259354 \\ \mathrm{H} & 4.761591 & 0.882359 & 5.258639 \\ \mathrm{H} & 4.210235 & -0.000692 & 6.686592\end{array}$

$\mathrm{CH}_{3}\left(\mathrm{CH}_{2}\right)_{7} \mathrm{OH}$

$\begin{array}{lrrr}\mathrm{C} & 0.002039 & 0.007360 & -0.001307 \\ \mathrm{C} & 0.000103 & 0.008724 & 1.526279 \\ \mathrm{C} & 1.411227 & 0.005727 & 2.113116 \\ \mathrm{C} & 1.422391 & 0.006986 & 3.641267 \\ \mathrm{C} & 2.832709 & 0.003411 & 4.229507 \\ \mathrm{C} & 2.837158 & 0.004624 & 5.757902 \\ \mathrm{C} & 4.248727 & -0.001919 & 6.343653 \\ \mathrm{C} & 4.229287 & 0.000470 & 7.859238 \\ \mathrm{O} & 5.583146 & -0.013325 & 8.329572 \\ \mathrm{H} & 4.801468 & 0.876990 & 5.995789 \\ \mathrm{H} & 4.791513 & -0.888259 & 5.999009 \\ \mathrm{H} & 5.576931 & -0.010531 & 9.293136 \\ \mathrm{H} & 3.716540 & 0.894598 & 8.228903 \\ \mathrm{H} & 3.698097 & -0.881914 & 8.231223 \\ \mathrm{H} & 2.288284 & -0.872551 & 6.119895 \\ \mathrm{H} & 2.296509 & 0.887443 & 6.118543 \\ \mathrm{H} & 3.379826 & 0.881184 & 3.865944 \\ \mathrm{H} & 3.374558 & -0.878268 & 3.867513 \\ \mathrm{H} & 0.876115 & -0.870868 & 4.006814 \\ \mathrm{H} & 0.881044 & 0.888490 & 4.005347 \\ \mathrm{H} & 1.956557 & 0.883669 & 1.746106 \\ \mathrm{H} & 1.952191 & -0.875547 & 1.747646 \\ \mathrm{H} & -0.543655 & -0.868347 & 1.893615 \\ \mathrm{H} & -0.539205 & 0.889196 & 1.892020 \\ \mathrm{H} & -1.013983 & 0.010254 & -0.389756 \\ \mathrm{H} & 0.519697 & -888626 \\ \mathrm{H} & 0.514100 & & \end{array}$


$\mathrm{ZnO}-\mathrm{CH}_{3} \mathrm{CH}_{2} \mathrm{OH}$

$\begin{array}{crrr}\mathrm{C} & 0.000000 & 0.000000 & 0.000000 \\ \mathrm{C} & 0.000000 & 0.000000 & 1.523320 \\ \mathrm{O} & 1.303288 & 0.000000 & 2.067883 \\ \mathrm{Zn} & 2.675045 & -0.989100 & 1.188396 \\ \mathrm{O} & 4.007116 & -1.966873 & 0.241635 \\ \mathrm{H} & 0.540458 & 0.872349 & -0.378423 \\ \mathrm{H} & 0.491102 & -0.902017 & -0.380791 \\ \mathrm{H} & -1.018647 & 0.024557 & -0.398150 \\ \mathrm{H} & -0.535934 & 0.890965 & 1.878875 \\ \mathrm{H} & -0.568210 & -0.873216 & 1.877975 \\ \mathrm{H} & 4.800547 & -1.439791 & 0.105127\end{array}$

$\mathrm{ZnO}-\mathrm{CH}_{3}\left(\mathrm{CH}_{2}\right)_{2} \mathrm{OH}$

$\begin{array}{lrrr}\mathrm{C} & -0.014977 & 0.001688 & 0.025037 \\ \mathrm{C} & 0.025248 & 0.037328 & 1.551314 \\ \mathrm{C} & 1.447365 & 0.010609 & 2.101828 \\ \mathrm{O} & 1.499238 & 0.062245 & 3.510536 \\ \mathrm{Zn} & 0.186221 & 1.067555 & 4.456686 \\ \mathrm{O} & -1.128069 & 2.052577 & 5.417434 \\ \mathrm{H} & -0.810233 & 2.931918 & 5.645306 \\ \mathrm{H} & 1.939889 & -0.910092 & 1.755061 \\ \mathrm{H} & 2.005347 & 0.852266 & 1.660271 \\ \mathrm{H} & -0.464887 & 0.949779 & 1.917493 \\ \mathrm{H} & -0.526716 & -0.813630 & 1.965099 \\ \mathrm{H} & 0.440681 & -0.916543 & -0.355222 \\ \mathrm{H} & 0.531261 & 0.848266 & -0.399964 \\ \mathrm{H} & -1.041050 & 0.044364 & -0.346066\end{array}$

$\mathrm{ZnO}-\mathrm{CH}_{3}\left(\mathrm{CH}_{2}\right)_{3} \mathrm{OH}$

$\mathrm{C}$
$\mathrm{C}$
$\mathrm{C}$
$\mathrm{C}$
$\mathrm{O}$
$\mathrm{Zn}$
$\mathrm{O}$
$\mathrm{H}$
$\mathrm{H}$
$\mathrm{H}$
2.315141

3.160725

3.025215

3.883849

3.703808

1.911483

0.078851

$-0.062164$

4.940861

3.651675
2.194956

1.085105

1.034394

$-0.048129$

$-0.128636$

$-0.120158$

$-0.116292$

0.197846

0.164574

$-1.011734$
6.395805

5.773726

4.252948

3.605635

2.206819

1.551231

1.062881

0.164402

3.820420

4.087436 


$\begin{array}{llll}\mathrm{H} & 1.974767 & 0.847460 & 3.984441 \\ \mathrm{H} & 3.297988 & 2.004831 & 3.821656 \\ \mathrm{H} & 4.213006 & 1.234565 & 6.038130 \\ \mathrm{H} & 2.863485 & 0.118845 & 6.194845 \\ \mathrm{H} & 2.410674 & 2.207687 & 7.484168 \\ \mathrm{H} & 2.622101 & 3.174811 & 6.019918 \\ \mathrm{H} & 1.257291 & 2.059609 & 6.153672\end{array}$

$\mathrm{ZnO}-\mathrm{CH}_{3}\left(\mathrm{CH}_{2}\right)_{4} \mathrm{OH}$

$\begin{array}{lrrr}\mathrm{C} & -0.018747 & 0.031117 & 0.025985 \\ \mathrm{C} & -0.000901 & 0.028589 & 1.553281 \\ \mathrm{C} & 1.416698 & -0.003428 & 2.125676 \\ \mathrm{C} & 1.432225 & 0.012707 & 3.653117 \\ \mathrm{C} & 2.833434 & 0.007596 & 4.256986 \\ \mathrm{O} & 2.823927 & 0.041658 & 5.668765 \\ \mathrm{Zn} & 1.508272 & 1.116895 & 6.528897 \\ \mathrm{O} & 0.229525 & 2.376200 & 7.159759 \\ \mathrm{H} & 3.391688 & 0.865873 & 3.849990 \\ \mathrm{H} & 3.358902 & -0.898572 & 3.922334 \\ \mathrm{H} & 0.910267 & 0.913415 & 4.007989 \\ \mathrm{H} & 0.881441 & -0.852791 & 4.040359 \\ \mathrm{H} & 1.977728 & 0.858947 & 1.746247 \\ \mathrm{H} & 1.932782 & -0.900458 & 1.763795 \\ \mathrm{H} & -0.517002 & 0.919459 & 1.927043 \\ \mathrm{H} & -0.558231 & -0.837442 & 1.926156 \\ \mathrm{H} & -1.040342 & 0.060607 & -0.360290 \\ \mathrm{H} & 0.514199 & 0.901472 & -0.366984 \\ \mathrm{H} & 0.465281 & -0.865394 & -0.371076 \\ \mathrm{H} & 0.112956 & 2.339875 & 8.114115\end{array}$

\section{$\mathrm{ZnO}-\mathrm{CH}_{3}\left(\mathrm{CH}_{2}\right)_{7} \mathrm{OH}$}

$\begin{array}{lrrr}\mathrm{C} & -2.791908 & 0.009020 & -2.636229 \\ \mathrm{C} & -1.371047 & 0.005065 & -2.075443 \\ \mathrm{C} & -1.349431 & 0.004366 & -0.547449 \\ \mathrm{C} & 0.062337 & 0.000486 & 0.037774 \\ \mathrm{C} & 0.066629 & 0.011774 & 1.565909 \\ \mathrm{C} & 1.471727 & 0.002682 & 2.167464 \\ \mathrm{C} & 1.451967 & 0.037303 & 3.694771 \\ \mathrm{C} & 2.838442 & 0.036425 & 4.329841 \\ \mathrm{O} & 2.795968 & 0.085292 & 5.740235 \\ \mathrm{Zn} & 1.481591 & 1.175698 & 6.566221\end{array}$




$\begin{array}{lrrr}\mathrm{O} & 0.207841 & 2.432676 & 7.188667 \\ \mathrm{H} & 3.408550 & 0.888673 & 3.927096 \\ \mathrm{H} & 3.369398 & -0.874529 & 4.018997 \\ \mathrm{H} & 0.922532 & 0.942358 & 4.027312 \\ \mathrm{H} & 0.891514 & -0.823384 & 4.078854 \\ \mathrm{H} & 2.032947 & 0.865898 & 1.790841 \\ \mathrm{H} & 2.005389 & -0.893475 & 1.830247 \\ \mathrm{H} & -0.470674 & 0.899617 & 1.920128 \\ \mathrm{H} & -0.488438 & -0.859097 & 1.934527 \\ \mathrm{H} & 0.610882 & 0.874987 & -0.332197 \\ \mathrm{H} & 0.601601 & -0.884628 & -0.320325 \\ \mathrm{H} & -0.196599 & 2.192748 & 8.027574 \\ \mathrm{H} & -1.895232 & -0.873434 & -0.180703 \\ \mathrm{H} & -1.890073 & 0.885330 & -0.180588 \\ \mathrm{H} & -0.828855 & 0.882927 & -2.443138 \\ \mathrm{H} & -0.832988 & -0.874809 & -2.444379 \\ \mathrm{H} & -2.791107 & 0.010450 & -3.728976 \\ \mathrm{H} & -3.344539 & -0.873767 & -2.302590 \\ \mathrm{H} & -3.340295 & 0.893581 & -2.300286 \\ & & & \end{array}$

$\left(\mathrm{ZnO}-\mathrm{CH}_{3} \mathrm{CH}_{2} \mathrm{OH}\right)_{2}$

$\begin{array}{crrr}\mathrm{C} & 3.050500 & -1.032844 & 4.797838 \\ \mathrm{C} & 2.615545 & -0.605701 & 3.405983 \\ \mathrm{O} & 1.352381 & 0.032809 & 3.431116 \\ \mathrm{Zn} & -0.001572 & 0.047437 & 4.919704 \\ \mathrm{O} & 0.041306 & 0.004570 & 6.843670 \\ \mathrm{C} & -0.483403 & -1.139413 & 7.478800 \\ \mathrm{C} & -0.316130 & -1.040345 & 8.988956 \\ \mathrm{Zn} & -0.010234 & 0.045319 & 1.958310 \\ \mathrm{O} & -1.373561 & 0.048503 & 3.442159 \\ \mathrm{O} & -0.049959 & -0.025534 & 0.035765 \\ \mathrm{H} & 2.336136 & -1.747823 & 5.217245 \\ \mathrm{H} & 3.104984 & -0.166641 & 5.462985 \\ \mathrm{H} & 4.034605 & -1.508773 & 4.769171 \\ \mathrm{H} & 3.363673 & 0.078394 & 2.985051 \\ \mathrm{H} & 2.579750 & -1.486220 & 2.751210 \\ \mathrm{H} & 0.836216 & -0.015384 & -0.338732 \\ \mathrm{H} & -2.064392 & 0.716772 & 3.445970 \\ \mathrm{H} & -0.721738 & -1.924112 & 9.490403 \\ \mathrm{H} & -0.836181 & -0.156929 & 9.369759 \\ \mathrm{H} & 0.743007 & -0.952568 & 9.246688 \\ \mathrm{H} & 0.020526 & -2.055334 & 7.129947 \\ \mathrm{H} & -1.553208 & -1.267020 & 7.248690\end{array}$


$\left(\mathrm{ZnO}-\mathrm{CH}_{3}\left(\mathrm{CH}_{2}\right)_{2} \mathrm{OH}\right)_{2}$

$\begin{array}{crrr}\mathrm{C} & -0.149204 & 0.057545 & 0.101358 \\ \mathrm{C} & -0.091559 & 0.054540 & 1.627939 \\ \mathrm{C} & 1.339013 & 0.075171 & 2.146494 \\ \mathrm{O} & 1.384306 & 0.008962 & 3.557065 \\ \mathrm{Zn} & -0.119178 & -0.013716 & 4.887514 \\ \mathrm{O} & -1.956854 & -0.570191 & 4.931304 \\ \mathrm{C} & -2.976122 & 0.399790 & 4.990969 \\ \mathrm{C} & -4.340084 & -0.252649 & 5.190533 \\ \mathrm{C} & -5.477015 & 0.766655 & 5.214870 \\ \mathrm{Zn} & 2.753467 & 0.722036 & 4.839172 \\ \mathrm{O} & 1.252677 & 0.710508 & 6.186771 \\ \mathrm{O} & 4.550230 & 1.428430 & 4.831115 \\ \mathrm{H} & -0.622930 & 0.925567 & 2.027884 \\ \mathrm{H} & -0.590158 & -0.838372 & 2.020226 \\ \mathrm{H} & 1.886713 & -0.775990 & 1.717577 \\ \mathrm{H} & 1.836371 & 0.989313 & 1.793377 \\ \mathrm{H} & 4.941371 & 1.403542 & 3.952450 \\ \mathrm{H} & 1.377685 & 0.251958 & 7.022290 \\ \mathrm{H} & -4.320893 & -0.819465 & 6.127348 \\ \mathrm{H} & -4.497818 & -0.973572 & 4.381419 \\ \mathrm{H} & -3.011951 & 0.997793 & 4.064321 \\ \mathrm{H} & -2.806544 & 1.112875 & 5.815143 \\ \mathrm{H} & -1.181387 & 0.046259 & -0.254325 \\ \mathrm{H} & 0.337229 & 0.947019 & -0.308175 \\ \mathrm{H} & 0.356228 & -0.820904 & -0.308916 \\ \mathrm{H} & -6.443838 & 5.363493 \\ \mathrm{H} & -5.340000 & 1.4896797 & 6.023939 \\ \mathrm{H} & -5.525016 & 1.322764 & 4.274345\end{array}$

$\left(\mathrm{ZnO}-\mathrm{CH}_{3}\left(\mathrm{CH}_{2}\right)_{3} \mathrm{OH}\right)_{2}$

$\begin{array}{crrr}\mathrm{C} & -1.746733 & 0.165320 & -1.897578 \\ \mathrm{C} & -1.647595 & 0.135837 & -0.373657 \\ \mathrm{C} & -0.200691 & 0.043142 & 0.109229 \\ \mathrm{C} & -0.084469 & 0.010215 & 1.626341 \\ \mathrm{O} & 1.266971 & -0.044373 & 2.041213 \\ \mathrm{Zn} & 2.036738 & -0.722323 & 3.763954 \\ \mathrm{O} & 1.515715 & -1.224775 & 5.553427 \\ \mathrm{Zn} & 2.890757 & -0.427118 & 0.923587 \\ \mathrm{O} & 3.679013 & -1.103804 & 2.657294\end{array}$




$\begin{array}{lrrr}\mathrm{O} & 3.455011 & -0.257373 & -0.902579 \\ \mathrm{C} & 3.833797 & -1.412072 & -1.616164 \\ \mathrm{C} & 4.543262 & -1.042327 & -2.913905 \\ \mathrm{C} & 4.962416 & -2.259137 & -3.737188 \\ \mathrm{C} & 5.686477 & -1.866519 & -5.023800 \\ \mathrm{H} & 0.267846 & -0.860936 & -0.300044 \\ \mathrm{H} & 0.369709 & 0.900837 & -0.267435 \\ \mathrm{H} & -0.563186 & 0.906219 & 2.044443 \\ \mathrm{H} & -0.634417 & -0.860000 & 2.010868 \\ \mathrm{H} & 0.677884 & -0.825966 & 5.807361 \\ \mathrm{H} & 4.548276 & -0.812300 & 2.944924 \\ \mathrm{H} & 5.426309 & -0.440368 & -2.668342 \\ \mathrm{H} & 3.875909 & -0.406352 & -3.507600 \\ \mathrm{H} & 2.954614 & -2.031279 & -1.862291 \\ \mathrm{H} & 4.507069 & -2.051326 & -1.021280 \\ \mathrm{H} & -2.215151 & -0.716626 & 0.014584 \\ \mathrm{H} & -2.109531 & 1.038097 & 0.040704 \\ \mathrm{H} & 5.613349 & -2.899795 & -3.132456 \\ \mathrm{H} & 4.076051 & -2.853430 & -3.983507 \\ \mathrm{H} & 5.976675 & -2.745162 & -5.604939 \\ \mathrm{H} & 5.046593 & -1.242754 & -5.654266 \\ \mathrm{H} & 6.592511 & -1.296016 & -4.800874 \\ \mathrm{H} & -2.785376 & 0.238374 & -2.228568 \\ \mathrm{H} & -1.202224 & 1.021612 & -2.304858 \\ \mathrm{H} & -1.318274 & -0.741306 & -2.333496 \\ & & & \end{array}$

$\left(\mathrm{ZnO}-\mathrm{CH}_{3}\left(\mathrm{CH}_{2}\right)_{4} \mathrm{OH}\right)_{2}$

$\begin{array}{crrr}\text { C } & 7.440741 & -0.830913 & 5.055583 \\ \mathrm{C} & 5.935156 & -1.034752 & 5.210809 \\ \mathrm{C} & 5.139504 & -0.352580 & 4.098697 \\ \mathrm{C} & 3.630883 & -0.554792 & 4.234004 \\ \mathrm{C} & 2.849455 & 0.126966 & 3.121725 \\ \mathrm{O} & 1.454333 & -0.034212 & 3.291191 \\ \mathrm{Zn} & 0.504085 & -1.353533 & 4.475701 \\ \mathrm{O} & 0.894662 & -2.407209 & 6.033199 \\ \mathrm{C} & -0.119870 & -3.046827 & 6.772237 \\ \mathrm{C} & 0.483279 & -3.935825 & 7.854841 \\ \mathrm{C} & -0.563409 & -4.621545 & 8.731245 \\ \mathrm{C} & 0.060138 & -5.524606 & 9.795688 \\ \mathrm{C} & -0.984794 & -6.192745 & 10.686574 \\ \mathrm{Zn} & 0.088797 & -0.072655 & 1.819640 \\ \mathrm{O} & -0.948174 & -1.282587 & 3.064376 \\ \mathrm{O} & 0.053599 & 0.413875 & -0.033930 \\ & & & \mathrm{~S} \\ & & & \end{array}$




$\begin{array}{lrrr}\mathrm{H} & 7.995538 & -1.319223 & 5.860234 \\ \mathrm{H} & 7.693050 & 0.233199 & 5.071350 \\ \mathrm{H} & 7.793468 & -1.243451 & 4.106138 \\ \mathrm{H} & 5.608290 & -0.642622 & 6.179661 \\ \mathrm{H} & 5.706755 & -2.105862 & 5.211610 \\ \mathrm{H} & 5.473589 & -0.739560 & 3.128602 \\ \mathrm{H} & 5.363957 & 0.720767 & 4.103419 \\ \mathrm{H} & 3.398133 & -1.626861 & 4.222664 \\ \mathrm{H} & 3.165379 & -0.286516 & 2.153693 \\ \mathrm{H} & 3.095911 & 1.197564 & 3.111538 \\ \mathrm{H} & 0.720633 & 1.070652 & -0.255117 \\ \mathrm{H} & -1.664145 & -6.812110 & 10.094184 \\ \mathrm{H} & -1.585557 & -5.444001 & 11.210607 \\ \mathrm{H} & -0.517357 & -6.833279 & 11.438470 \\ \mathrm{H} & 0.667969 & -6.291582 & 9.303730 \\ \mathrm{H} & 0.742774 & -4.931335 & 10.413620 \\ \mathrm{H} & -1.235607 & -5.218747 & 8.103218 \\ \mathrm{H} & -1.183592 & -3.862384 & 9.223226 \\ \mathrm{H} & 1.115237 & -4.691039 & 7.372838 \\ \mathrm{H} & 1.140028 & -3.318788 & 8.479128 \\ \mathrm{H} & -0.785393 & -2.312045 & 7.255356 \\ \mathrm{H} & -0.759325 & -3.667466 & 6.123188 \\ \mathrm{H} & -1.858174 & -1.082294 & 3.299153 \\ \mathrm{H} & 3.287269 & -0.158501 & 5.197085 \\ & & & \end{array}$

$\left(\mathrm{ZnO}-\mathrm{CH}_{3}\left(\mathrm{CH}_{2}\right)_{7} \mathrm{OH}\right)_{2}$

$\begin{array}{crrr}\mathrm{C} & 7.678743 & -1.798857 & 12.515366 \\ \mathrm{C} & 7.388427 & -2.510534 & 11.195454 \\ \mathrm{C} & 6.590557 & -1.639309 & 10.226557 \\ \mathrm{C} & 6.285517 & -2.336523 & 8.901415 \\ \mathrm{C} & 5.489007 & -1.457511 & 7.938423 \\ \mathrm{C} & 5.176829 & -2.151246 & 6.612851 \\ \mathrm{C} & 4.390682 & -1.259082 & 5.652988 \\ \mathrm{C} & 4.071752 & -1.951112 & 4.336365 \\ \mathrm{O} & 3.372113 & -1.088565 & 3.456265 \\ \mathrm{Zn} & 1.744347 & -0.016804 & 3.956467 \\ \mathrm{O} & 0.740374 & 0.311747 & 5.554972 \\ \mathrm{O} & 1.307642 & -0.124479 & 2.003838 \\ \mathrm{Zn} & 2.619663 & -1.625591 & 1.665798 \\ \mathrm{O} & 2.642671 & -2.982438 & 0.317553 \\ \mathrm{C} & -0.004864 & -0.078341 & 1.473259 \\ \mathrm{C} & 0.003808 & -0.070264 & -0.048684 \\ \mathrm{C} & -1.407954 & -0.024766 & -0.632758 \\ & & & \mathrm{~S} \\ & & & \end{array}$




\begin{tabular}{|c|c|c|c|}
\hline $\mathrm{C}$ & -1.422112 & -0.000752 & -2.160861 \\
\hline $\mathrm{C}$ & -2.836125 & 0.042729 & -2.739195 \\
\hline $\mathrm{C}$ & -2.858682 & 0.066404 & -4.266950 \\
\hline $\mathrm{C}$ & -4.273896 & 0.110124 & -4.842581 \\
\hline $\mathrm{C}$ & -4.283981 & 0.129798 & -6.370014 \\
\hline $\mathrm{H}$ & 0.579485 & 0.796592 & -0.392234 \\
\hline $\mathrm{H}$ & 0.521935 & -0.968475 & -0.405048 \\
\hline $\mathrm{H}$ & -0.029966 & 0.870199 & 5.415555 \\
\hline $\mathrm{H}$ & -0.511255 & 0.827086 & 1.833581 \\
\hline $\mathrm{H}$ & -0.591825 & -0.938814 & 1.825362 \\
\hline $\mathrm{H}$ & 4.962650 & -0.349242 & 5.438439 \\
\hline $\mathrm{H}$ & 3.451104 & -0.949122 & 6.126729 \\
\hline $\mathrm{H}$ & 3.379457 & -3.595833 & 0.392433 \\
\hline $\mathrm{H}$ & 5.007814 & -2.272094 & 3.860601 \\
\hline $\mathrm{H}$ & 3.481872 & -2.856768 & 4.536891 \\
\hline $\mathrm{H}$ & -1.973859 & -0.895839 & -0.282041 \\
\hline $\mathrm{H}$ & -1.929425 & 0.862911 & -0.255169 \\
\hline $\mathrm{H}$ & -0.859055 & 0.871280 & -2.514107 \\
\hline $\mathrm{H}$ & -0.901265 & -0.887393 & -2.540809 \\
\hline $\mathrm{H}$ & -3.398386 & -0.829112 & -2.383663 \\
\hline $\mathrm{H}$ & -3.356099 & 0.929403 & -2.356867 \\
\hline $\mathrm{H}$ & 4.606575 & -3.066524 & 6.810475 \\
\hline $\mathrm{H}$ & 6.115142 & -2.460033 & 6.137133 \\
\hline $\mathrm{H}$ & 6.051591 & -0.537520 & 7.739967 \\
\hline $\mathrm{H}$ & 4.550157 & -1.154773 & 8.416688 \\
\hline $\mathrm{H}$ & 5.724882 & -3.258197 & 9.098330 \\
\hline $\mathrm{H}$ & 7.226483 & -2.636376 & 8.424648 \\
\hline $\mathrm{H}$ & 7.149001 & -0.716164 & 10.029456 \\
\hline $\mathrm{H}$ & 5.649063 & -1.340233 & 10.702845 \\
\hline $\mathrm{H}$ & 6.832616 & -3.433949 & 11.390950 \\
\hline $\mathrm{H}$ & 8.331512 & -2.805185 & 10.722542 \\
\hline $\mathrm{H}$ & 8.254682 & -2.433174 & 13.193486 \\
\hline $\mathrm{H}$ & 8.251991 & -0.883038 & 12.345350 \\
\hline $\mathrm{H}$ & 6.749782 & -1.521835 & 13.021558 \\
\hline $\mathrm{H}$ & -2.296980 & 0.938009 & -4.624363 \\
\hline $\mathrm{H}$ & -2.339915 & -0.820385 & -4.650507 \\
\hline $\mathrm{H}$ & -4.835364 & -0.758821 & -4.482642 \\
\hline $\mathrm{H}$ & -4.789571 & 0.997847 & -4.460626 \\
\hline $\mathrm{H}$ & -5.302639 & 0.169192 & -6.762913 \\
\hline $\mathrm{H}$ & -3.743258 & 1.000968 & -6.750505 \\
\hline $\mathrm{H}$ & -3.802140 & -0.764928 & -6.773811 \\
\hline
\end{tabular}

$\left(\mathrm{ZnO}-\mathrm{CH}_{3} \mathrm{CH}_{2} \mathrm{OH}\right)_{4}$ 


\begin{tabular}{|c|c|c|c|}
\hline $\mathrm{C}$ & -2.938304 & -1.390889 & 6.361371 \\
\hline $\mathrm{C}$ & -2.223902 & -0.503734 & 7.368082 \\
\hline $\mathrm{O}$ & -1.372541 & 0.419617 & 6.711627 \\
\hline $\mathrm{Zn}$ & -0.033821 & 1.500965 & 7.751567 \\
\hline $\mathrm{O}$ & -0.049020 & 2.292725 & 9.522659 \\
\hline $\mathrm{Zn}$ & 0.088683 & -0.352452 & 5.503966 \\
\hline $\mathrm{O}$ & 0.760531 & -2.188072 & 6.217290 \\
\hline $\mathrm{Zn}$ & 1.265813 & -2.725352 & 4.341447 \\
\hline $\mathrm{O}$ & 1.151730 & -4.225826 & 2.974790 \\
\hline $\mathrm{C}$ & -0.052599 & -4.421120 & 2.254055 \\
\hline $\mathrm{C}$ & -1.199874 & -4.739310 & 3.198665 \\
\hline $\mathrm{O}$ & 0.106248 & -1.149980 & 3.647408 \\
\hline $\mathrm{O}$ & 1.260285 & 1.168763 & 6.228927 \\
\hline $\mathrm{C}$ & 2.642964 & 0.947733 & 6.440679 \\
\hline $\mathrm{C}$ & 3.234558 & 2.002823 & 7.361016 \\
\hline $\mathrm{O}$ & 3.191053 & -2.467010 & 3.644182 \\
\hline $\mathrm{Zn}$ & 2.862796 & -3.648039 & 2.057270 \\
\hline $\mathrm{O}$ & 3.362451 & -3.700629 & 0.198001 \\
\hline $\mathrm{C}$ & 4.377718 & -2.503375 & 4.411898 \\
\hline $\mathrm{C}$ & 5.588630 & -2.174719 & 3.553233 \\
\hline $\mathrm{H}$ & -3.586692 & -2.112958 & 6.865785 \\
\hline $\mathrm{H}$ & -3.550586 & -0.786968 & 5.686820 \\
\hline $\mathrm{H}$ & -2.209475 & -1.943964 & 5.760191 \\
\hline $\mathrm{H}$ & -2.964493 & 0.041881 & 7.966448 \\
\hline $\mathrm{H}$ & -1.650354 & -1.132893 & 8.063629 \\
\hline $\mathrm{H}$ & 0.712837 & 2.866150 & 9.651298 \\
\hline $\mathrm{H}$ & 2.751947 & 1.961458 & 8.342058 \\
\hline $\mathrm{H}$ & 3.083788 & 3.001305 & 6.942102 \\
\hline $\mathrm{H}$ & 4.307396 & 1.843452 & 7.501011 \\
\hline $\mathrm{H}$ & 2.812465 & -0.046565 & 6.876208 \\
\hline $\mathrm{H}$ & 3.166333 & 0.971921 & 5.475267 \\
\hline $\mathrm{H}$ & 0.494007 & -0.602398 & 2.957603 \\
\hline $\mathrm{H}$ & 6.509717 & -2.201796 & 4.142229 \\
\hline $\mathrm{H}$ & 5.680898 & -2.898828 & 2.737553 \\
\hline $\mathrm{H}$ & 5.484291 & -1.177357 & 3.117744 \\
\hline $\mathrm{H}$ & 4.513021 & -3.493107 & 4.869570 \\
\hline $\mathrm{H}$ & 4.304317 & -1.777757 & 5.232370 \\
\hline $\mathrm{H}$ & 0.096722 & -2.750215 & 6.628878 \\
\hline $\mathrm{H}$ & -1.351043 & -3.912021 & 3.899842 \\
\hline $\mathrm{H}$ & -0.980091 & -5.643362 & 3.772751 \\
\hline $\mathrm{H}$ & -2.131540 & -4.895222 & 2.647695 \\
\hline $\mathrm{H}$ & -0.300661 & -3.526156 & 1.667347 \\
\hline $\mathrm{H}$ & 0.077540 & -5.247123 & 1.543519 \\
\hline $\mathrm{H}$ & 4.028096 & -3.037641 & -0.009917 \\
\hline
\end{tabular}


$\left(\mathrm{ZnO}-\mathrm{CH}_{3}\left(\mathrm{CH}_{2}\right)_{2} \mathrm{OH}\right)_{4}$

\begin{tabular}{|c|c|c|c|}
\hline $\mathrm{C}$ & -1.578350 & 0.140652 & -0.359632 \\
\hline $\mathrm{C}$ & -0.140041 & 0.122794 & 0.154207 \\
\hline $\mathrm{C}$ & -0.070743 & 0.104439 & 1.675171 \\
\hline $\mathrm{O}$ & 1.271200 & 0.035091 & 2.122959 \\
\hline $\mathrm{Zn}$ & 2.388049 & -1.498707 & 1.379588 \\
\hline $\mathrm{O}$ & 3.252211 & -1.528110 & 3.236396 \\
\hline $\mathrm{C}$ & 3.646375 & -2.769410 & 3.789521 \\
\hline $\mathrm{C}$ & 4.049961 & -2.638086 & 5.251041 \\
\hline $\mathrm{C}$ & 4.495757 & -3.975648 & 5.839236 \\
\hline $\mathrm{Zn}$ & 1.743704 & -0.445600 & 4.030705 \\
\hline $\mathrm{O}$ & 0.737986 & -0.137181 & 5.642535 \\
\hline $\mathrm{O}$ & 1.363132 & -3.246544 & 0.968209 \\
\hline $\mathrm{Zn}$ & 2.885487 & -3.851187 & -0.215177 \\
\hline $\mathrm{Zn}$ & 4.943799 & -5.556332 & -1.328959 \\
\hline $\mathrm{O}$ & 6.484586 & -6.016901 & -2.394187 \\
\hline $\mathrm{O}$ & 3.548537 & -1.892941 & -0.227100 \\
\hline $\mathrm{O}$ & 3.174865 & -4.813846 & -1.978121 \\
\hline $\mathrm{C}$ & 3.081513 & -4.063966 & -3.175317 \\
\hline $\mathrm{C}$ & 1.697831 & -3.450073 & -3.335505 \\
\hline $\mathrm{C}$ & 1.571455 & -2.652469 & -4.632018 \\
\hline $\mathrm{O}$ & 4.416620 & -5.016713 & 0.535459 \\
\hline $\mathrm{C}$ & 4.220341 & -5.979178 & 1.554537 \\
\hline $\mathrm{C}$ & 5.545883 & -6.512258 & 2.081224 \\
\hline $\mathrm{C}$ & 5.349875 & -7.536417 & 3.197125 \\
\hline $\mathrm{H}$ & 0.405154 & 0.998650 & -0.212096 \\
\hline $\mathrm{H}$ & 0.383380 & -0.764411 & -0.224303 \\
\hline $\mathrm{H}$ & -0.548605 & 1.010802 & 2.071017 \\
\hline $\mathrm{H}$ & -0.649958 & -0.753925 & 2.048695 \\
\hline $\mathrm{H}$ & 1.202360 & -0.436997 & 6.429851 \\
\hline $\mathrm{H}$ & 3.195570 & -2.244150 & 5.814039 \\
\hline $\mathrm{H}$ & 4.857363 & -1.902777 & 5.327961 \\
\hline $\mathrm{H}$ & 2.831985 & -3.505360 & 3.711925 \\
\hline $\mathrm{H}$ & 4.497525 & -3.168794 & 3.218622 \\
\hline $\mathrm{H}$ & 4.491926 & -1.754259 & -0.096676 \\
\hline $\mathrm{H}$ & 6.099268 & -6.962785 & 1.249020 \\
\hline $\mathrm{H}$ & 6.139703 & -5.666638 & 2.444274 \\
\hline $\mathrm{H}$ & 3.615007 & -6.821483 & 1.186731 \\
\hline $\mathrm{H}$ & 3.663960 & -5.526845 & 2.387238 \\
\hline $\mathrm{H}$ & 0.510394 & -3.165421 & 0.530044 \\
\hline $\mathrm{H}$ & 1.502627 & -2.799617 & -2.474106 \\
\hline $\mathrm{H}$ & 0.954538 & -4.253682 & -3.310560 \\
\hline $\mathrm{H}$ & 3.833537 & -3.260881 & -3.192460 \\
\hline
\end{tabular}




$\begin{array}{lrrr}\mathrm{H} & 3.287816 & -4.716390 & -4.034838 \\ \mathrm{H} & 7.269152 & -6.138107 & -1.850684 \\ \mathrm{H} & 4.774980 & -3.874879 & 6.890107 \\ \mathrm{H} & 3.694559 & -4.717462 & 5.775429 \\ \mathrm{H} & 5.362066 & -4.368086 & 5.298866 \\ \mathrm{H} & -1.608794 & 0.156322 & -1.451171 \\ \mathrm{H} & -2.125335 & -0.744170 & -0.022201 \\ \mathrm{H} & -2.112031 & 1.023528 & 0.002600 \\ \mathrm{H} & 6.308254 & -7.901453 & 3.572350 \\ \mathrm{H} & 4.806643 & -7.097927 & 4.039050 \\ \mathrm{H} & 4.779082 & -8.398952 & 2.842320 \\ \mathrm{H} & 0.576218 & -2.215040 & -4.734347 \\ \mathrm{H} & 2.300806 & -1.838504 & -4.662182 \\ \mathrm{H} & 1.747165 & -3.292446 & -5.501096\end{array}$

$\left(\mathrm{ZnO}-\mathrm{CH}_{3}\left(\mathrm{CH}_{2}\right)_{3} \mathrm{OH}\right)_{4}$

$\begin{array}{crrr}\mathrm{C} & 4.857222 & -3.898620 & 7.335790 \\ \mathrm{C} & 4.506118 & -4.018569 & 5.853867 \\ \mathrm{C} & 4.051939 & -2.688118 & 5.254174 \\ \mathrm{C} & 3.668215 & -2.810560 & 3.786345 \\ \mathrm{O} & 3.278165 & -1.565306 & 3.236487 \\ \mathrm{Zn} & 2.414591 & -1.529639 & 1.381147 \\ \mathrm{O} & 3.524471 & -1.932492 & -0.260126 \\ \mathrm{Zn} & 2.776358 & -3.870475 & -0.283940 \\ \mathrm{Zn} & 4.806739 & -5.589253 & -1.418760 \\ \mathrm{O} & 6.343397 & -6.037177 & -2.489967 \\ \mathrm{O} & 1.296456 & 0.007514 & 2.124953 \\ \mathrm{Zn} & 1.779227 & -0.473237 & 4.030323 \\ \mathrm{O} & 0.772852 & -0.166386 & 5.639897 \\ \mathrm{C} & -0.059275 & 0.041552 & 1.712510 \\ \mathrm{C} & -0.173690 & 0.009755 & 0.194772 \\ \mathrm{C} & -1.622507 & -0.030347 & -0.291208 \\ \mathrm{C} & -1.721326 & -0.209404 & -1.804785 \\ \mathrm{O} & 1.352535 & -3.254174 & 0.992069 \\ \mathrm{O} & 3.042066 & -4.812895 & -2.060104 \\ \mathrm{C} & 3.028270 & -4.071901 & -3.268605 \\ \mathrm{C} & 1.750100 & -3.260456 & -3.427359 \\ \mathrm{C} & 1.697505 & -2.531705 & -4.770130 \\ \mathrm{C} & 0.449727 & -1.663983 & -4.918103 \\ \mathrm{O} & 4.294407 & -5.056434 & 0.447732 \\ \mathrm{C} & 4.118106 & -6.005334 & 1.483405 \\ \mathrm{C} & 5.454873 & -6.459210 & 2.054571 \\ \mathrm{C} & 5.302187 & -7.477687 & 3.183440\end{array}$




\begin{tabular}{|c|c|c|c|}
\hline $\mathrm{C}$ & 6.643420 & -7.844875 & 3.816140 \\
\hline $\mathrm{H}$ & 0.337762 & 0.884960 & -0.221576 \\
\hline $\mathrm{H}$ & 0.360625 & -0.877287 & -0.171311 \\
\hline $\mathrm{H}$ & -0.541317 & 0.950262 & 2.097166 \\
\hline $\mathrm{H}$ & -0.611400 & -0.815336 & 2.127657 \\
\hline $\mathrm{H}$ & 1.231964 & -0.464245 & 6.430910 \\
\hline $\mathrm{H}$ & 3.186190 & -2.309317 & 5.812786 \\
\hline $\mathrm{H}$ & 4.851377 & -1.944575 & 5.350616 \\
\hline $\mathrm{H}$ & 2.856246 & -3.546575 & 3.691753 \\
\hline $\mathrm{H}$ & 4.527038 & -3.202142 & 3.222357 \\
\hline $\mathrm{H}$ & 4.474766 & -1.838023 & -0.140606 \\
\hline $\mathrm{H}$ & 6.058263 & -6.890313 & 1.245659 \\
\hline $\mathrm{H}$ & 5.993301 & -5.577835 & 2.422651 \\
\hline $\mathrm{H}$ & 3.563440 & -6.882725 & 1.118782 \\
\hline $\mathrm{H}$ & 3.518534 & -5.561342 & 2.289861 \\
\hline $\mathrm{H}$ & 0.467212 & -3.156955 & 0.628338 \\
\hline $\mathrm{H}$ & 1.684234 & -2.531728 & -2.608928 \\
\hline $\mathrm{H}$ & 0.889270 & -3.933116 & -3.335575 \\
\hline $\mathrm{H}$ & 3.889121 & -3.389015 & -3.315933 \\
\hline $\mathrm{H}$ & 3.120736 & -4.762615 & -4.117804 \\
\hline $\mathrm{H}$ & 7.134142 & -6.151476 & -1.954141 \\
\hline $\mathrm{H}$ & 3.712817 & -4.762803 & 5.725021 \\
\hline $\mathrm{H}$ & 5.377223 & -4.385341 & 5.299718 \\
\hline $\mathrm{H}$ & -2.146431 & -0.853999 & 0.206029 \\
\hline $\mathrm{H}$ & -2.132012 & 0.891902 & 0.006331 \\
\hline $\mathrm{H}$ & 4.637030 & -7.067890 & 3.951328 \\
\hline $\mathrm{H}$ & 4.817293 & -8.380193 & 2.796706 \\
\hline $\mathrm{H}$ & 2.590726 & -1.906721 & -4.876724 \\
\hline $\mathrm{H}$ & 1.731013 & -3.269570 & -5.578519 \\
\hline $\mathrm{H}$ & 5.184082 & -4.856583 & 7.746952 \\
\hline $\mathrm{H}$ & 5.662979 & -3.175105 & 7.487383 \\
\hline $\mathrm{H}$ & 3.991760 & -3.562359 & 7.913371 \\
\hline $\mathrm{H}$ & -2.761814 & -0.223235 & -2.138158 \\
\hline $\mathrm{H}$ & -1.211135 & 0.603454 & -2.328943 \\
\hline $\mathrm{H}$ & -1.257292 & -1.150847 & -2.111863 \\
\hline $\mathrm{H}$ & 0.415682 & -1.178001 & -5.896066 \\
\hline $\mathrm{H}$ & -0.458370 & -2.263994 & -4.809762 \\
\hline $\mathrm{H}$ & 0.429191 & -0.882218 & -4.154401 \\
\hline $\mathrm{H}$ & 6.522293 & -8.582346 & 4.613097 \\
\hline $\mathrm{H}$ & 7.324617 & -8.264505 & 3.070538 \\
\hline $\mathrm{H}$ & 7.122872 & -6.961104 & 4.246499 \\
\hline
\end{tabular}

$\left(\mathrm{ZnO}-\mathrm{CH}_{3}\left(\mathrm{CH}_{2}\right)_{4} \mathrm{OH}\right)_{4}$

C

0.232599

0.063406

$-0.287814$ 


\begin{tabular}{|c|c|c|c|}
\hline $\mathrm{C}$ & 0.213966 & 0.025381 & 1.239391 \\
\hline $\mathrm{C}$ & 1.621848 & 0.034539 & 1.833421 \\
\hline $\mathrm{C}$ & 1.636521 & -0.020014 & 3.361188 \\
\hline $\mathrm{C}$ & 3.055664 & -0.018856 & 3.912063 \\
\hline $\mathrm{O}$ & 3.085642 & -0.106712 & 5.326589 \\
\hline $\mathrm{Zn}$ & 2.403457 & 1.348684 & 6.539987 \\
\hline $\mathrm{O}$ & 1.036299 & 2.701504 & 6.543594 \\
\hline $\mathrm{Zn}$ & 4.924184 & -0.082095 & 6.223410 \\
\hline $\mathrm{O}$ & 6.265496 & -1.597999 & 6.223640 \\
\hline $\mathrm{Zn}$ & 7.291457 & -0.710654 & 4.673666 \\
\hline $\mathrm{Zn}$ & 8.513723 & -2.460794 & 2.731948 \\
\hline $\mathrm{O}$ & 9.348166 & -3.950807 & 1.850404 \\
\hline $\mathrm{O}$ & 4.010418 & 0.992197 & 7.693181 \\
\hline $\mathrm{C}$ & 4.743291 & 2.040415 & 8.303382 \\
\hline $\mathrm{C}$ & 6.114853 & 1.564543 & 8.760947 \\
\hline $\mathrm{C}$ & 6.900120 & 2.648495 & 9.498205 \\
\hline $\mathrm{C}$ & 8.326046 & 2.213453 & 9.832061 \\
\hline $\mathrm{C}$ & 9.098008 & 3.271540 & 10.616813 \\
\hline $\mathrm{O}$ & 6.268665 & 0.999775 & 5.071667 \\
\hline $\mathrm{O}$ & 9.179340 & -1.249160 & 4.208593 \\
\hline $\mathrm{C}$ & 10.031437 & -1.732377 & 5.233763 \\
\hline $\mathrm{C}$ & 10.118923 & -0.757424 & 6.400080 \\
\hline $\mathrm{C}$ & 11.072073 & -1.248358 & 7.490424 \\
\hline $\mathrm{C}$ & 11.118549 & -0.328803 & 8.710044 \\
\hline $\mathrm{C}$ & 12.095366 & -0.824025 & 9.774801 \\
\hline $\mathrm{O}$ & 6.717699 & -1.553108 & 2.871222 \\
\hline $\mathrm{C}$ & 6.311175 & -0.705273 & 1.808823 \\
\hline $\mathrm{C}$ & 5.661996 & -1.492896 & 0.679221 \\
\hline $\mathrm{C}$ & 5.190826 & -0.592262 & -0.461465 \\
\hline $\mathrm{C}$ & 4.494334 & -1.362485 & -1.582635 \\
\hline $\mathrm{C}$ & 4.029269 & -0.447711 & -2.713387 \\
\hline $\mathrm{H}$ & 5.984628 & 0.690920 & 9.409838 \\
\hline $\mathrm{H}$ & 6.683315 & 1.232645 & 7.882374 \\
\hline $\mathrm{H}$ & 4.185865 & 2.421421 & 9.169797 \\
\hline $\mathrm{H}$ & 4.871073 & 2.882628 & 7.607241 \\
\hline $\mathrm{H}$ & 0.324284 & 2.505478 & 5.927263 \\
\hline $\mathrm{H}$ & 1.094423 & 0.841824 & 3.770364 \\
\hline $\mathrm{H}$ & 1.122348 & -0.923481 & 3.707863 \\
\hline $\mathrm{H}$ & 3.568027 & 0.892650 & 3.570912 \\
\hline $\mathrm{H}$ & 3.601398 & -0.873482 & 3.487203 \\
\hline $\mathrm{H}$ & 5.908719 & -2.476303 & 6.058795 \\
\hline $\mathrm{H}$ & 6.381061 & -2.229409 & 0.299264 \\
\hline $\mathrm{H}$ & 4.812039 & -2.052106 & 1.087450 \\
\hline $\mathrm{H}$ & 7.167765 & -0.142100 & 1.408075 \\
\hline $\mathrm{H}$ & 5.594072 & 0.036616 & 2.184325 \\
\hline
\end{tabular}




\begin{tabular}{|c|c|c|c|}
\hline $\mathrm{H}$ & 6.774553 & 1.673882 & 5.535893 \\
\hline $\mathrm{H}$ & 9.114614 & -0.619506 & 6.822257 \\
\hline $\mathrm{H}$ & 10.451696 & 0.216315 & 6.022781 \\
\hline $\mathrm{H}$ & 9.674289 & -2.702564 & 5.607930 \\
\hline $\mathrm{H}$ & 11.036706 & -1.894833 & 4.823086 \\
\hline $\mathrm{H}$ & 8.733727 & -4.416812 & 1.275220 \\
\hline $\mathrm{H}$ & 2.146634 & 0.936932 & 1.497116 \\
\hline $\mathrm{H}$ & 2.182383 & -0.821161 & 1.436742 \\
\hline $\mathrm{H}$ & 6.936836 & 3.556822 & 8.884621 \\
\hline $\mathrm{H}$ & 6.373805 & 2.914032 & 10.422243 \\
\hline $\mathrm{H}$ & 4.502267 & 0.164533 & -0.066059 \\
\hline $\mathrm{H}$ & 6.048136 & -0.049507 & -0.877449 \\
\hline $\mathrm{H}$ & 10.767546 & -2.251300 & 7.812627 \\
\hline $\mathrm{H}$ & 12.080859 & -1.344862 & 7.072307 \\
\hline $\mathrm{H}$ & -0.314536 & -0.871780 & 1.578714 \\
\hline $\mathrm{H}$ & -0.345272 & 0.885619 & 1.622522 \\
\hline $\mathrm{H}$ & 8.292829 & 1.281504 & 10.406749 \\
\hline $\mathrm{H}$ & 8.855678 & 1.989376 & 8.900168 \\
\hline $\mathrm{H}$ & 11.400922 & 0.681411 & 8.393486 \\
\hline $\mathrm{H}$ & 10.115257 & -0.256391 & 9.140927 \\
\hline $\mathrm{H}$ & 5.179577 & -2.119311 & -1.979354 \\
\hline $\mathrm{H}$ & 3.635338 & -1.901688 & -1.168457 \\
\hline $\mathrm{H}$ & -0.778396 & 0.055793 & -0.701709 \\
\hline $\mathrm{H}$ & 0.736624 & 0.965241 & -0.646918 \\
\hline $\mathrm{H}$ & 0.767552 & -0.801605 & -0.690851 \\
\hline $\mathrm{H}$ & 10.120353 & 2.945805 & 10.825071 \\
\hline $\mathrm{H}$ & 9.151414 & 4.209680 & 10.057087 \\
\hline $\mathrm{H}$ & 8.610616 & 3.479794 & 11.573244 \\
\hline $\mathrm{H}$ & 12.113635 & -0.158001 & 10.640911 \\
\hline $\mathrm{H}$ & 11.814347 & -1.821401 & 10.124380 \\
\hline $\mathrm{H}$ & 13.111646 & -0.883562 & 9.375330 \\
\hline $\mathrm{H}$ & 3.534047 & -1.012214 & -3.507074 \\
\hline $\mathrm{H}$ & 3.322156 & 0.299881 & -2.342360 \\
\hline $\mathrm{H}$ & 4.875854 & 0.083598 & -3.157278 \\
\hline
\end{tabular}

$\left(\mathrm{ZnO}-\mathrm{CH}_{3}\left(\mathrm{CH}_{2}\right)_{7} \mathrm{OH}\right)_{4}$

$\begin{array}{lrrr}\mathrm{C} & -4.488118 & 0.129177 & -6.159621 \\ \mathrm{C} & -4.488488 & 0.091402 & -4.632260 \\ \mathrm{C} & -3.073742 & 0.073823 & -4.055496 \\ \mathrm{C} & -3.038775 & 0.043995 & -2.528256 \\ \mathrm{C} & -1.614619 & -0.010015 & -1.975680 \\ \mathrm{C} & -1.558009 & -0.012691 & -0.448723 \\ \mathrm{C} & -0.127199 & -0.074886 & 0.085555\end{array}$




\begin{tabular}{|c|c|c|c|}
\hline $\mathrm{C}$ & -0.060181 & -0.097289 & 1.606366 \\
\hline $\mathrm{O}$ & 1.280517 & -0.208808 & 2.052722 \\
\hline $\mathrm{Zn}$ & 2.386094 & -1.733150 & 1.290955 \\
\hline $\mathrm{O}$ & 3.241467 & -1.794520 & 3.147538 \\
\hline $\mathrm{C}$ & 3.615651 & -3.053128 & 3.680173 \\
\hline $\mathrm{C}$ & 4.066046 & -2.948228 & 5.130175 \\
\hline $\mathrm{C}$ & 4.530034 & -4.293786 & 5.687114 \\
\hline $\mathrm{C}$ & 4.954954 & -4.225155 & 7.153385 \\
\hline $\mathrm{C}$ & 5.477143 & -5.562507 & 7.677126 \\
\hline $\mathrm{C}$ & 5.871546 & -5.519840 & 9.152548 \\
\hline $\mathrm{C}$ & 6.421290 & -6.852597 & 9.659329 \\
\hline $\mathrm{C}$ & 6.790753 & -6.809505 & 11.140523 \\
\hline $\mathrm{Zn}$ & 1.762301 & -0.691870 & 3.950428 \\
\hline $\mathrm{O}$ & 0.771978 & -0.389458 & 5.560991 \\
\hline $\mathrm{O}$ & 1.292322 & -3.425407 & 0.914814 \\
\hline $\mathrm{Zn}$ & 2.628520 & -4.058149 & -0.444093 \\
\hline $\mathrm{Zn}$ & 4.306779 & -6.126636 & -1.640599 \\
\hline $\mathrm{O}$ & 5.819438 & -6.652177 & -2.701655 \\
\hline $\mathrm{O}$ & 3.467153 & -2.177264 & -0.358948 \\
\hline $\mathrm{O}$ & 2.769689 & -4.957415 & -2.262151 \\
\hline $\mathrm{C}$ & 2.974208 & -4.144945 & -3.408855 \\
\hline $\mathrm{C}$ & 1.834180 & -3.156230 & -3.607245 \\
\hline $\mathrm{C}$ & 2.054036 & -2.249108 & -4.817975 \\
\hline $\mathrm{C}$ & 0.951503 & -1.204615 & -4.983969 \\
\hline $\mathrm{C}$ & 1.174567 & -0.280548 & -6.180763 \\
\hline $\mathrm{C}$ & 0.080290 & 0.775856 & -6.326091 \\
\hline $\mathrm{C}$ & 0.319959 & 1.734053 & -7.492137 \\
\hline $\mathrm{C}$ & -0.770176 & 2.797711 & -7.604701 \\
\hline $\mathrm{O}$ & 3.804027 & -5.593045 & 0.208269 \\
\hline $\mathrm{C}$ & 3.747751 & -6.365481 & 1.389176 \\
\hline $\mathrm{C}$ & 5.135144 & -6.640427 & 1.953982 \\
\hline $\mathrm{C}$ & 5.088982 & -7.515271 & 3.206201 \\
\hline $\mathrm{C}$ & 6.460426 & -7.710038 & 3.851539 \\
\hline $\mathrm{C}$ & 6.415869 & -8.633239 & 5.068293 \\
\hline $\mathrm{C}$ & 7.772221 & -8.788580 & 5.753782 \\
\hline $\mathrm{C}$ & 7.726865 & -9.729958 & 6.956431 \\
\hline $\mathrm{C}$ & 9.083405 & -9.864996 & 7.644621 \\
\hline $\mathrm{H}$ & 0.447392 & 0.783913 & -0.279335 \\
\hline $\mathrm{H}$ & 0.367633 & -0.978266 & -0.297173 \\
\hline $\mathrm{H}$ & -0.506717 & 0.822471 & 2.005731 \\
\hline $\mathrm{H}$ & -0.663838 & -0.937660 & 1.981332 \\
\hline $\mathrm{H}$ & 1.223089 & -0.675843 & 6.360381 \\
\hline $\mathrm{H}$ & 3.232291 & -2.565945 & 5.733127 \\
\hline $\mathrm{H}$ & 4.879004 & -2.216577 & 5.195260 \\
\hline $\mathrm{H}$ & 2.778682 & -3.763842 & 3.618118 \\
\hline
\end{tabular}




\begin{tabular}{|c|c|c|c|}
\hline $\mathrm{H}$ & 4.437393 & -3.469203 & 3.079558 \\
\hline $\mathrm{H}$ & 4.423062 & -2.095104 & -0.292121 \\
\hline $\mathrm{H}$ & 5.741177 & -7.130594 & 1.181905 \\
\hline $\mathrm{H}$ & 5.618205 & -5.683228 & 2.182449 \\
\hline $\mathrm{H}$ & 3.241743 & -7.322652 & 1.196669 \\
\hline $\mathrm{H}$ & 3.150685 & -5.836287 & 2.143691 \\
\hline $\mathrm{H}$ & 0.374334 & -3.337845 & 0.642848 \\
\hline $\mathrm{H}$ & 1.745487 & -2.541335 & -2.701995 \\
\hline $\mathrm{H}$ & 0.896213 & -3.712324 & -3.718286 \\
\hline $\mathrm{H}$ & 3.917531 & -3.585806 & -3.322531 \\
\hline $\mathrm{H}$ & 3.059768 & -4.784103 & -4.297557 \\
\hline $\mathrm{H}$ & 6.611779 & -6.800296 & -2.177040 \\
\hline $\mathrm{H}$ & -2.126668 & -0.869834 & -0.068474 \\
\hline $\mathrm{H}$ & -2.051893 & 0.887812 & -0.065878 \\
\hline $\mathrm{H}$ & -1.046688 & 0.847528 & -2.356529 \\
\hline $\mathrm{H}$ & -1.115570 & -0.909667 & -2.355582 \\
\hline $\mathrm{H}$ & -3.600669 & -0.826462 & -2.168624 \\
\hline $\mathrm{H}$ & -3.548245 & 0.932153 & -2.135995 \\
\hline $\mathrm{H}$ & -2.529077 & 0.956489 & -4.411439 \\
\hline $\mathrm{H}$ & -2.538534 & -0.800709 & -4.445240 \\
\hline $\mathrm{H}$ & -5.030585 & -0.795216 & -4.286058 \\
\hline $\mathrm{H}$ & -5.027194 & 0.962109 & -4.243032 \\
\hline $\mathrm{H}$ & -5.504021 & 0.130947 & -6.561459 \\
\hline $\mathrm{H}$ & -3.980635 & 1.026705 & -6.524592 \\
\hline $\mathrm{H}$ & -3.965384 & -0.739867 & -6.569483 \\
\hline $\mathrm{H}$ & 3.723385 & -5.028930 & 5.581564 \\
\hline $\mathrm{H}$ & 5.371589 & -4.660645 & 5.086933 \\
\hline $\mathrm{H}$ & 5.733206 & -3.461990 & 7.270415 \\
\hline $\mathrm{H}$ & 4.102450 & -3.903560 & 7.763112 \\
\hline $\mathrm{H}$ & 4.709164 & -6.331892 & 7.532109 \\
\hline $\mathrm{H}$ & 6.345253 & -5.867576 & 7.080134 \\
\hline $\mathrm{H}$ & 6.624415 & -4.737311 & 9.305478 \\
\hline $\mathrm{H}$ & 4.998496 & -5.237791 & 9.753028 \\
\hline $\mathrm{H}$ & 5.675018 & -7.636683 & 9.490246 \\
\hline $\mathrm{H}$ & 7.303183 & -7.125034 & 9.069085 \\
\hline $\mathrm{H}$ & 7.186031 & -7.769238 & 11.481814 \\
\hline $\mathrm{H}$ & 7.551048 & -6.046665 & 11.330261 \\
\hline $\mathrm{H}$ & 5.917008 & -6.570018 & 11.752982 \\
\hline $\mathrm{H}$ & 4.409439 & -7.065479 & 3.939986 \\
\hline $\mathrm{H}$ & 4.668780 & -8.494788 & 2.949084 \\
\hline $\mathrm{H}$ & 7.156873 & -8.120561 & 3.110743 \\
\hline $\mathrm{H}$ & 6.860176 & -6.733320 & 4.150484 \\
\hline $\mathrm{H}$ & 5.688799 & -8.244220 & 5.791188 \\
\hline $\mathrm{H}$ & 6.052725 & -9.620482 & 4.758404 \\
\hline $\mathrm{H}$ & 8.505404 & -9.163436 & 5.029325 \\
\hline
\end{tabular}




$\begin{array}{lrrr}\mathrm{H} & 8.129421 & -7.803697 & 6.079430 \\ \mathrm{H} & 6.985208 & -9.361572 & 7.673194 \\ \mathrm{H} & 7.382605 & -10.716308 & 6.627281 \\ \mathrm{H} & 9.030848 & -10.536892 & 8.504648 \\ \mathrm{H} & 9.831693 & -10.262885 & 6.953301 \\ \mathrm{H} & 9.440328 & -8.893331 & 7.998009 \\ \mathrm{H} & 3.018322 & -1.737914 & -4.714095 \\ \mathrm{H} & 2.116714 & -2.859728 & -5.726326 \\ \mathrm{H} & -0.015384 & -1.710738 & -5.091055 \\ \mathrm{H} & 0.890980 & -0.600205 & -4.071242 \\ \mathrm{H} & 2.145268 & 0.218087 & -6.073170 \\ \mathrm{H} & 1.227862 & -0.877810 & -7.098874 \\ \mathrm{H} & -0.889189 & 0.280653 & -6.459342 \\ \mathrm{H} & 0.010878 & 1.352419 & -5.395223 \\ \mathrm{H} & 1.293718 & 2.218897 & -7.364382 \\ \mathrm{H} & 0.373561 & 1.162215 & -8.424925 \\ \mathrm{H} & -0.581281 & 3.477849 & -8.438659 \\ \mathrm{H} & -1.749296 & 2.336755 & -7.762963 \\ \mathrm{H} & -0.826654 & 3.394746 & -6.689993\end{array}$

$(\mathrm{ZnO})_{12}$ in ethanol

$\begin{array}{crrr}\mathrm{O} & -0.527942 & -2.182852 & -2.203074 \\ \mathrm{Zn} & -2.245207 & -1.483867 & -1.423526 \\ \mathrm{Zn} & -2.557528 & 1.278697 & 0.160822 \\ \mathrm{O} & -2.481153 & 0.444791 & 1.900682 \\ \mathrm{Zn} & -0.736714 & 0.855207 & 2.815614 \\ \mathrm{O} & -0.310184 & -1.088853 & 3.115678 \\ \mathrm{Zn} & 1.377946 & -1.545089 & 2.273471 \\ \mathrm{O} & 2.839751 & -0.351056 & 1.603507 \\ \mathrm{Zn} & 2.245211 & 1.483858 & 1.423515 \\ \mathrm{O} & 0.527946 & 2.182843 & 2.203062 \\ \mathrm{Zn} & 0.207664 & 2.965464 & 0.373948 \\ \mathrm{O} & 2.069000 & 2.553062 & -0.267924 \\ \mathrm{Zn} & 1.817149 & 1.461795 & -1.845916 \\ \mathrm{Zn} & -1.377943 & 1.545078 & -2.273483 \\ \mathrm{O} & -1.422973 & 2.724191 & -0.633674 \\ \mathrm{Zn} & 0.736718 & -0.855216 & -2.815625 \\ \mathrm{Zn} & 2.557534 & -1.278705 & -0.160832 \\ \mathrm{Zn} & -0.207659 & -2.965470 & -0.373960 \\ \mathrm{O} & -2.068996 & -2.553070 & 0.267913 \\ \mathrm{Zn} & -1.817144 & -1.461804 & 1.845905 \\ \mathrm{O} & 2.481157 & -0.444800 & -1.900692\end{array}$




$$
\begin{aligned}
& \mathrm{O} \\
& \mathrm{O} \\
& \mathrm{O}
\end{aligned}
$$

0.310189

$-2.839749$

1.422978
1.088844

0.351046

$-2.724200$
$-3.115687$

$-1.603516$

0.633662

$(\mathrm{ZnO})_{12}$ in 1-propanol

$\begin{array}{crrr}\mathrm{O} & -0.523275 & -2.165024 & -2.182332 \\ \mathrm{Zn} & -2.245836 & -1.481746 & -1.404347 \\ \mathrm{Zn} & -2.556997 & 1.287828 & 0.159168 \\ \mathrm{O} & -2.480569 & 0.437753 & 1.891402 \\ \mathrm{Zn} & -0.741802 & 0.849126 & 2.814250 \\ \mathrm{O} & -0.31534 & -1.093006 & 3.113063 \\ \mathrm{Zn} & 1.378168 & -1.553165 & 2.276256 \\ \mathrm{O} & 2.831376 & -0.354150 & 1.601430 \\ \mathrm{Zn} & 2.245824 & 1.481741 & 1.404331 \\ \mathrm{O} & 0.523257 & 2.165020 & 2.182301 \\ \mathrm{Zn} & 0.208747 & 2.992055 & 0.372878 \\ \mathrm{O} & 2.064299 & 2.566039 & -0.274879 \\ \mathrm{Zn} & 1.818987 & 1.468131 & -1.847869 \\ \mathrm{Zn} & -1.378155 & 1.553128 & -2.276242 \\ \mathrm{O} & -1.418808 & 2.730058 & -0.635119 \\ \mathrm{Zn} & 0.741814 & -0.849151 & -2.814258 \\ \mathrm{Zn} & 2.556992 & -1.287790 & -0.159160 \\ \mathrm{Zn} & -0.208739 & -2.992045 & -0.372910 \\ \mathrm{O} & -2.064295 & -2.566056 & 0.274855 \\ \mathrm{Zn} & -1.818960 & -1.468160 & 1.847850 \\ \mathrm{O} & 2.480596 & -0.437786 & -1.891432 \\ \mathrm{O} & 0.311545 & 1.092982 & -3.113058 \\ \mathrm{O} & -2.831388 & 0.354151 & -1.601405 \\ \mathrm{O} & 1.418805 & -2.730040 & 0.635097\end{array}$

$(\mathrm{ZnO})_{12}$ in 1-butanol

$\begin{array}{lrrr}\mathrm{O} & -0.521897 & -2.159490 & -2.174356 \\ \mathrm{Zn} & -2.247114 & -1.482186 & -1.398403 \\ \mathrm{Zn} & -2.559762 & 1.290900 & 0.157725 \\ \mathrm{O} & -2.479010 & 0.436172 & 1.886923 \\ \mathrm{Zn} & -0.743013 & 0.848308 & 2.813422 \\ \mathrm{O} & -0.310815 & -1.093194 & 3.106651 \\ \mathrm{Zn} & 1.381720 & -1.560993 & 2.279329 \\ \mathrm{O} & 2.825675 & -0.354882 & 1.601132 \\ \mathrm{Zn} & 2.247114 & 1.482178 & 1.398396 \\ \mathrm{O} & 0.521897 & 2.159484 & 2.174346\end{array}$




$\begin{array}{crrr}\mathrm{Zn} & 0.209157 & 3.001618 & 0.373335 \\ \mathrm{O} & 2.061513 & 2.568533 & -0.277317 \\ \mathrm{Zn} & 1.820950 & 1.469326 & -1.849059 \\ \mathrm{Zn} & -1.381717 & 1.560982 & -2.279340 \\ \mathrm{O} & -1.417092 & 2.730058 & -0.633699 \\ \mathrm{Zn} & 0.743016 & -0.848316 & -2.813432 \\ \mathrm{Zn} & 2.559765 & -1.290907 & -0.157736 \\ \mathrm{Zn} & -0.209154 & -3.001628 & -0.373348 \\ \mathrm{O} & -2.061509 & -2.568544 & 0.277308 \\ \mathrm{Zn} & -1.820943 & -1.469336 & 1.849048 \\ \mathrm{O} & 2.479016 & -0.436183 & -1.886936 \\ \mathrm{O} & 0.310821 & 1.093187 & -3.106660 \\ \mathrm{O} & -2.825673 & 0.354873 & -1.601144 \\ \mathrm{O} & 1.417095 & -2.730067 & 0.633688\end{array}$

$(\mathrm{ZnO})_{12}$ in 1-pentanol

$\begin{array}{crrr}\mathrm{O} & -0.519167 & -2.124384 & -2.218591 \\ \mathrm{Zn} & -2.244503 & -1.463206 & -1.433202 \\ \mathrm{Zn} & -2.587358 & 1.304630 & 0.177898 \\ \mathrm{O} & -2.464869 & 0.400374 & 1.877499 \\ \mathrm{Zn} & -0.741479 & 0.796344 & 2.823792 \\ \mathrm{O} & -0.319086 & -1.152606 & 3.093341 \\ \mathrm{Zn} & 1.365777 & -1.603717 & 2.243856 \\ \mathrm{O} & 2.807128 & -0.381420 & 1.591283 \\ \mathrm{Zn} & 2.244519 & 1.463186 & 1.433197 \\ \mathrm{O} & 0.519186 & 2.124366 & 2.218593 \\ \mathrm{Zn} & 0.204130 & 3.001010 & 0.435911 \\ \mathrm{O} & 2.053012 & 2.573455 & -0.227195 \\ \mathrm{Zn} & 1.826700 & 1.509745 & -1.824012 \\ \mathrm{Zn} & -1.365734 & 1.603745 & -2.243835 \\ \mathrm{O} & -1.419720 & 2.743103 & -0.578769 \\ \mathrm{Zn} & 0.741458 & -0.796341 & -2.823836 \\ \mathrm{Zn} & 2.587271 & -1.304625 & -0.177925 \\ \mathrm{Zn} & -0.204127 & -3.001030 & -0.435907 \\ \mathrm{O} & -2.053013 & -2.573485 & 0.227189 \\ \mathrm{Zn} & -1.826671 & -1.509766 & 1.823994 \\ \mathrm{O} & 2.464858 & -0.400409 & -1.877550 \\ \mathrm{O} & 0.319103 & 1.152622 & -3.093361 \\ \mathrm{O} & -2.807066 & 0.381412 & -1.591305 \\ \mathrm{O} & 1.419700 & -2.743107 & 0.578805 \\ & & & \end{array}$

$(\mathrm{ZnO})_{12}$ in 1-octanol 
$\mathrm{O}$
$\mathrm{Zn}$
$\mathrm{Zn}$
$\mathrm{O}$
$\mathrm{Zn}$
$\mathrm{O}$
$\mathrm{Zn}$
$\mathrm{O}$
$\mathrm{Zn}$
$\mathrm{O}$
$\mathrm{Zn}$
$\mathrm{O}$
$\mathrm{Zn}$
$\mathrm{Zn}$
$\mathrm{O}$
$\mathrm{Zn}$
$\mathrm{Zn}$
$\mathrm{Zn}$
$\mathrm{O}$
$\mathrm{Zn}$
$\mathrm{O}$
$\mathrm{O}$
$\mathrm{O}$
$\mathrm{O}$

$(\mathrm{ZnO})_{12}-(\mathrm{ZnO})_{12}$ in ethanol
$-0.515870$

$-2.236531$

$-2.607658$

$-2.474202$

$-0.742236$

$-0.310250$

1.373433

2.776062

2.236544

0.515881

0.199141

2.039026

1.832265

$-1.373438$

$-1.433515$

0.742248

2.607682

$-0.199134$

$-2.039021$

$-1.832277$

2.474199

0.310245

$-2.776063$

1.433521
$-2.170936$

$-1.491622$

1.323707

0.445563

0.859820

$-1.081263$

$-1.595930$

$-0.354717$

1.491613

2.170921

3.027967

2.570510

1.456682

1.595921

2.747124

$-0.859833$

$-1.323727$

$-3.027973$

$-2.570508$

$-1.456686$

$-0.445559$

1.081247

0.354707

$-2.747136$
$-2.164287$

$-1.389235$

0.163353

1.875076

2.804279

3.078168

2.261396

1.577858

1.389207

2.164269

0.376843

$-0.285023$

$-1.847331$

$-2.261410$

$-0.612192$

$-2.804290$

$-0.163356$

$-0.376856$

0.285007

1.847320

$-1.875065$

$-3.078180$

$-1.577861$

0.612181
$\mathrm{O}$

$\mathrm{Zn}$

$\mathrm{O}$

$\mathrm{Zn}$

$\mathrm{Zn}$

$\mathrm{O}$

$\mathrm{Zn}$

$\mathrm{O}$

$\mathrm{Zn}$

$\mathrm{O}$

$\mathrm{Zn}$

$\mathrm{O}$

$\mathrm{Zn}$

$\mathrm{O}$

$\mathrm{Zn}$

$\mathrm{O}$

$\mathrm{Zn}$
$-0.182492$

$-0.127621$

0.987598

2.287464

5.011927

5.574364

5.406437

4.061988

2.666615

1.097137

2.388415

3.396968

4.967013

6.367367

6.236912

4.631540

5.921805
0.022357

$-1.966681$

$-3.316457$

$-4.179773$

$-2.491010$

$-0.584169$

$-0.519676$

0.831949

1.690511

1.121948

0.058701

0.110355

0.892123

1.815112

1.661551

1.104649

$-0.107165$
$-0.219256$

$-0.034649$

$-0.866893$

0.409706

0.417229

0.333507

2.334313

2.996762

1.855596

2.949653

4.067267

5.862008

4.827281

5.927989

7.859054

8.936916

9.905544 


\begin{tabular}{|c|c|c|c|}
\hline $\mathrm{O}$ & 7.537066 & 0.798125 & 9.135278 \\
\hline $\mathrm{Zn}$ & 8.652351 & -0.551649 & 8.303126 \\
\hline $\mathrm{Zn}$ & 7.644761 & 0.413746 & 5.321210 \\
\hline $\mathrm{O}$ & 6.358536 & -0.579027 & 4.152496 \\
\hline $\mathrm{Zn}$ & 6.136171 & -2.577035 & 4.201493 \\
\hline $\mathrm{O}$ & 5.127605 & -2.628696 & 2.406799 \\
\hline $\mathrm{Zn}$ & 3.557525 & -3.410464 & 3.441460 \\
\hline $\mathrm{O}$ & 2.157112 & -4.333402 & 2.340769 \\
\hline $\mathrm{Zn}$ & 0.879743 & -2.932044 & 2.947672 \\
\hline $\mathrm{O}$ & 2.166029 & -1.939308 & 4.116324 \\
\hline $\mathrm{Zn}$ & 3.118184 & -1.998670 & 5.934481 \\
\hline $\mathrm{O}$ & 2.950344 & -1.934197 & 7.935290 \\
\hline $\mathrm{Zn}$ & 4.337120 & -2.893816 & 8.888970 \\
\hline $\mathrm{Zn}$ & 5.858004 & -4.208923 & 6.413053 \\
\hline $\mathrm{O}$ & 4.462592 & -3.350302 & 5.271968 \\
\hline $\mathrm{Zn}$ & -0.037425 & 0.070578 & 1.784730 \\
\hline $\mathrm{O}$ & -0.415728 & -1.901111 & 1.956100 \\
\hline $\mathrm{Zn}$ & 1.491127 & 0.628804 & -1.005598 \\
\hline $\mathrm{Zn}$ & 4.187609 & 0.375374 & -0.620278 \\
\hline $\mathrm{O}$ & 2.859594 & 1.768721 & -0.072937 \\
\hline $\mathrm{Zn}$ & 3.512728 & -0.027337 & 7.851586 \\
\hline $\mathrm{O}$ & 5.638103 & -2.012521 & 10.170243 \\
\hline $\mathrm{Zn}$ & 7.033645 & -3.147256 & 9.274124 \\
\hline $\mathrm{O}$ & 8.707210 & -2.540686 & 8.487773 \\
\hline $\mathrm{Zn}$ & 8.562155 & -2.588918 & 6.483781 \\
\hline $\mathrm{O}$ & 8.940496 & -0.617226 & 6.312378 \\
\hline $\mathrm{O}$ & 5.665103 & -4.287169 & 8.341587 \\
\hline $\mathrm{O}$ & 7.427482 & -3.640327 & 5.319004 \\
\hline $\mathrm{O}$ & 2.886713 & -0.505939 & -1.901621 \\
\hline $\mathrm{Zn}$ & 2.603046 & -2.411327 & -1.636999 \\
\hline $\mathrm{O}$ & 3.893047 & -3.623063 & -0.667948 \\
\hline
\end{tabular}

$(\mathrm{ZnO})_{12}-(\mathrm{ZnO})_{12}$ in 1-propanol

$\begin{array}{crrr}\mathrm{O} & 0.010580 & -0.138754 & 0.013027 \\ \mathrm{Zn} & 1.521625 & 1.171387 & 0.016640 \\ \mathrm{O} & 1.890901 & 2.778955 & -1.000162 \\ \mathrm{Zn} & 1.711818 & 4.385419 & 0.186150 \\ \mathrm{Zn} & -1.280002 & 5.736150 & 0.081184 \\ \mathrm{O} & -3.036894 & 4.787801 & -0.055368 \\ \mathrm{Zn} & -2.929638 & 4.658681 & 1.950093 \\ \mathrm{O} & -3.381224 & 3.112731 & 3.022641 \\ \mathrm{Zn} & -3.198151 & 1.519025 & 1.849295 \\ \mathrm{O} & -1.732993 & 0.634121 & 2.868673\end{array}$




\begin{tabular}{|c|c|c|c|}
\hline $\mathrm{Zn}$ & -1.230991 & -0.346560 & 4.596782 \\
\hline $\mathrm{O}$ & 0.596515 & -0.975646 & 3.726554 \\
\hline $\mathrm{Zn}$ & 0.382571 & -2.612115 & 4.855551 \\
\hline $\mathrm{Zn}$ & 3.377705 & -2.131074 & 5.803913 \\
\hline $\mathrm{O}$ & 3.829113 & -0.585148 & 4.731263 \\
\hline $\mathrm{Zn}$ & 3.646018 & 1.008523 & 5.904640 \\
\hline $\mathrm{Zn}$ & 3.338775 & -0.576018 & 8.753921 \\
\hline $\mathrm{O}$ & 3.484751 & -2.260243 & 7.809334 \\
\hline $\mathrm{Zn}$ & 1.727899 & -3.208643 & 7.672613 \\
\hline $\mathrm{O}$ & 1.896677 & -3.489279 & 5.677422 \\
\hline $\mathrm{Zn}$ & -0.024730 & -0.014334 & 2.018381 \\
\hline $\mathrm{O}$ & 1.748969 & 1.207480 & 2.004225 \\
\hline $\mathrm{Zn}$ & 1.678967 & 2.874151 & 3.157156 \\
\hline $\mathrm{O}$ & -0.148581 & 3.503227 & 4.027339 \\
\hline $\mathrm{Zn}$ & -1.840232 & 2.401643 & 4.104201 \\
\hline $\mathrm{O}$ & -1.301118 & 1.320136 & 5.749674 \\
\hline $\mathrm{Zn}$ & 0.472637 & 2.541898 & 5.735521 \\
\hline $\mathrm{O}$ & 0.437352 & 2.666302 & 7.740877 \\
\hline $\mathrm{Zn}$ & -1.073737 & 1.356203 & 7.737251 \\
\hline $\mathrm{O}$ & -1.442955 & -0.251388 & 8.754037 \\
\hline $\mathrm{Zn}$ & -1.263883 & -1.857840 & 7.567718 \\
\hline $\mathrm{O}$ & -1.366459 & -2.043736 & 5.638625 \\
\hline $\mathrm{Zn}$ & -1.492272 & 0.747912 & -0.829929 \\
\hline $\mathrm{O}$ & -3.246788 & 1.352386 & -0.071702 \\
\hline $\mathrm{Zn}$ & -2.890896 & 3.103575 & -0.999962 \\
\hline $\mathrm{O}$ & -1.404615 & 2.321014 & -2.103396 \\
\hline $\mathrm{Zn}$ & 0.166264 & 3.407831 & -1.826160 \\
\hline $\mathrm{O}$ & 0.269821 & 5.212002 & -0.947210 \\
\hline $\mathrm{Zn}$ & 2.288201 & 0.125914 & 3.649602 \\
\hline $\mathrm{O}$ & 2.180900 & 1.893444 & 4.885214 \\
\hline $\mathrm{O}$ & 3.694684 & 1.175164 & 7.825636 \\
\hline $\mathrm{Zn}$ & 1.940191 & 1.779668 & 8.583879 \\
\hline $\mathrm{Zn}$ & 0.281670 & -0.880269 & 9.580040 \\
\hline $\mathrm{O}$ & 0.178130 & -2.684434 & 8.701063 \\
\hline $\mathrm{O}$ & 1.852514 & 0.206590 & 9.857352 \\
\hline $\mathrm{O}$ & 1.814487 & 4.571292 & 2.115251 \\
\hline $\mathrm{Zn}$ & 0.065425 & 5.139641 & 2.898285 \\
\hline $\mathrm{O}$ & -1.448629 & 6.016844 & 2.076362 \\
\hline
\end{tabular}

$(\mathrm{ZnO})_{12}-(\mathrm{ZnO})_{12}$ in 1-butanol

$\begin{array}{crrr}\mathrm{O} & 0.001716 & -0.026878 & -0.046488 \\ \mathrm{Zn} & -1.638681 & -1.243786 & 0.126084 \\ \mathrm{O} & -1.713385 & -1.136820 & 2.133687\end{array}$




\begin{tabular}{|c|c|c|c|}
\hline $\mathrm{Zn}$ & -2.610357 & 0.419161 & 3.254157 \\
\hline $\mathrm{O}$ & -3.625664 & -0.906831 & 4.365652 \\
\hline $\mathrm{Zn}$ & -5.555838 & -0.866006 & 4.190923 \\
\hline $\mathrm{O}$ & -6.615306 & -2.407145 & 3.416251 \\
\hline $\mathrm{Zn}$ & -7.529526 & -1.019706 & 2.295466 \\
\hline $\mathrm{O}$ & -6.730419 & 0.514547 & 3.328418 \\
\hline $\mathrm{Zn}$ & -5.738102 & 1.608351 & 2.080679 \\
\hline $\mathrm{O}$ & -3.754520 & 1.775533 & 2.279774 \\
\hline $\mathrm{Zn}$ & -3.708517 & 1.693654 & 0.160667 \\
\hline $\mathrm{O}$ & -5.714908 & 1.805163 & 0.091892 \\
\hline $\mathrm{Zn}$ & -6.482964 & 0.190496 & -0.645473 \\
\hline $\mathrm{O}$ & -5.727597 & -1.286342 & -1.766661 \\
\hline $\mathrm{Zn}$ & -6.604428 & -2.539104 & -0.459884 \\
\hline $\mathrm{Zn}$ & -5.620614 & -3.732496 & 2.418992 \\
\hline $\mathrm{O}$ & -3.621972 & -3.965240 & 2.448665 \\
\hline $\mathrm{Zn}$ & -2.880835 & -2.351160 & 3.202770 \\
\hline $\mathrm{Zn}$ & -3.740633 & -3.802186 & 0.448696 \\
\hline $\mathrm{O}$ & -5.748203 & -4.026627 & 0.429309 \\
\hline $\mathrm{Zn}$ & -0.033440 & 0.087194 & 2.067992 \\
\hline $\mathrm{O}$ & -0.908036 & 1.565498 & 3.117059 \\
\hline $\mathrm{Zn}$ & -2.129370 & 3.011569 & 2.125589 \\
\hline $\mathrm{O}$ & -1.129500 & 4.524534 & 2.988308 \\
\hline $\mathrm{Zn}$ & 0.043099 & 3.163171 & 3.857047 \\
\hline $\mathrm{O}$ & 1.969375 & 3.091531 & 3.976389 \\
\hline $\mathrm{Zn}$ & 2.810587 & 4.342592 & 2.641846 \\
\hline $\mathrm{O}$ & 3.916117 & 2.880868 & 1.828224 \\
\hline $\mathrm{Zn}$ & 3.740461 & 2.836416 & -0.100039 \\
\hline $\mathrm{O}$ & 2.796916 & 4.206710 & -1.215072 \\
\hline $\mathrm{Zn}$ & 1.786849 & 5.524414 & -0.223812 \\
\hline $\mathrm{O}$ & 1.905071 & 5.810744 & 1.767956 \\
\hline $\mathrm{Zn}$ & -0.105370 & 5.577212 & 1.729530 \\
\hline $\mathrm{O}$ & -0.213875 & 5.749111 & -0.269732 \\
\hline $\mathrm{Zn}$ & -0.948955 & 4.132475 & -1.034100 \\
\hline $\mathrm{Zn}$ & -1.126800 & 1.343859 & -1.011932 \\
\hline $\mathrm{O}$ & -2.837078 & 0.209917 & -0.878065 \\
\hline $\mathrm{Zn}$ & -3.800792 & -1.369822 & -1.634162 \\
\hline $\mathrm{O}$ & -2.653628 & -2.742437 & -0.747318 \\
\hline $\mathrm{Zn}$ & 1.985220 & 0.189446 & 0.142320 \\
\hline $\mathrm{Zn}$ & 1.759231 & 2.654362 & -1.991124 \\
\hline $\mathrm{O}$ & -0.171448 & 2.683415 & -2.169317 \\
\hline $\mathrm{O}$ & 1.972878 & -0.004458 & 2.129506 \\
\hline $\mathrm{Zn}$ & 2.733024 & 1.617076 & 2.859994 \\
\hline $\mathrm{O}$ & 2.947019 & 1.290013 & -1.122874 \\
\hline $\mathrm{O}$ & -2.015832 & 2.899364 & 0.121365 \\
\hline $\mathrm{O}$ & -7.690335 & -1.068358 & 0.367767 \\
\hline
\end{tabular}


$(\mathrm{ZnO})_{12}-(\mathrm{ZnO})_{12}$ in 1-pentanol

\begin{tabular}{|c|c|c|c|}
\hline $\mathrm{O}$ & -1.726892 & -3.201438 & -4.316856 \\
\hline $\mathrm{Zn}$ & -3.468590 & -2.483173 & -3.625435 \\
\hline $\mathrm{Zn}$ & -5.164419 & -3.746391 & -1.676849 \\
\hline $\mathrm{O}$ & -5.321000 & -2.698104 & 0.185219 \\
\hline $\mathrm{Zn}$ & -6.832605 & -3.959247 & 0.555959 \\
\hline $\mathrm{O}$ & -8.478229 & -3.305239 & 1.340658 \\
\hline $\mathrm{Zn}$ & -8.582855 & -1.317159 & 1.124961 \\
\hline $\mathrm{O}$ & -10.370905 & -1.400114 & 0.185513 \\
\hline $\mathrm{Zn}$ & -10.392146 & -0.613683 & -1.584109 \\
\hline $\mathrm{O}$ & -9.155842 & 0.801668 & -2.280725 \\
\hline $\mathrm{Zn}$ & -7.594937 & 0.821839 & -1.136794 \\
\hline $\mathrm{Zn}$ & -5.524864 & -0.688090 & -0.025826 \\
\hline $\mathrm{O}$ & -7.266577 & 0.030128 & 0.665597 \\
\hline $\mathrm{Zn}$ & -0.410606 & -1.854151 & -4.776207 \\
\hline $\mathrm{Zn}$ & -1.398498 & -3.993078 & -2.514451 \\
\hline $\mathrm{Zn}$ & -0.102825 & -2.794326 & 0.220965 \\
\hline $\mathrm{O}$ & -1.673866 & -2.213262 & 1.196411 \\
\hline $\mathrm{Zn}$ & -1.844996 & -0.238704 & 0.884197 \\
\hline $\mathrm{O}$ & -3.513430 & -0.360720 & -0.218677 \\
\hline $\mathrm{Zn}$ & -3.829029 & 0.575137 & -1.974402 \\
\hline $\mathrm{O}$ & -5.851058 & 0.225794 & -1.924181 \\
\hline $\mathrm{Zn}$ & -5.699786 & -0.699509 & -3.703345 \\
\hline $\mathrm{Zn}$ & -8.890624 & -0.376925 & -3.872175 \\
\hline $\mathrm{O}$ & -10.442277 & -1.550376 & -3.355587 \\
\hline $\mathrm{Zn}$ & -10.109775 & -3.461493 & -3.264524 \\
\hline $\mathrm{O}$ & -9.992680 & -4.479820 & -1.539904 \\
\hline $\mathrm{Zn}$ & -10.014397 & -3.368214 & 0.042088 \\
\hline $\mathrm{O}$ & 1.377436 & -1.771201 & -3.836737 \\
\hline $\mathrm{Zn}$ & 1.398686 & -2.557582 & -2.067093 \\
\hline $\mathrm{O}$ & 1.448822 & -1.620877 & -0.295627 \\
\hline $\mathrm{Zn}$ & 1.116346 & 0.290250 & -0.386687 \\
\hline $\mathrm{O}$ & -0.572014 & 1.096702 & 0.327829 \\
\hline $\mathrm{Zn}$ & -0.844070 & 1.838159 & -1.517804 \\
\hline $\mathrm{O}$ & 0.999236 & 1.308503 & -2.111343 \\
\hline $\mathrm{Zn}$ & 1.020944 & 0.196904 & -3.693338 \\
\hline $\mathrm{Zn}$ & -2.160839 & 0.787961 & -4.207219 \\
\hline $\mathrm{O}$ & -2.458748 & 1.894538 & -2.583829 \\
\hline $\mathrm{O}$ & -0.515225 & 0.133927 & -4.991910 \\
\hline $\mathrm{O}$ & -3.672457 & -0.473160 & -3.836466 \\
\hline $\mathrm{O}$ & 0.162372 & -3.972926 & -1.370476 \\
\hline $\mathrm{O}$ & -3.142389 & -3.397043 & -1.727070 \\
\hline
\end{tabular}




$\begin{array}{crrr}\mathrm{Zn} & -3.293672 & -2.471742 & 0.052096 \\ \mathrm{O} & -5.480025 & -2.810535 & -3.432576 \\ \mathrm{Zn} & -7.148467 & -2.932552 & -4.535444 \\ \mathrm{O} & -7.319599 & -0.957991 & -4.847644 \\ \mathrm{O} & -8.421437 & -4.267965 & -3.979073 \\ \mathrm{Zn} & -8.149372 & -5.009450 & -2.133455 \\ \mathrm{O} & -6.534689 & -5.065814 & -1.067436\end{array}$

$(\mathrm{ZnO})_{12}-(\mathrm{ZnO})_{12}$ in 1-octanol

\begin{tabular}{|c|c|c|c|}
\hline $\mathrm{O}$ & -0.385662 & -2.349306 & -2.371176 \\
\hline $\mathrm{Zn}$ & -2.113432 & -1.775060 & -1.514753 \\
\hline $\mathrm{O}$ & -2.841681 & 0.009882 & -1.618114 \\
\hline $\mathrm{Zn}$ & -3.096950 & 0.978563 & 0.097734 \\
\hline $\mathrm{Zn}$ & -3.744084 & 1.326059 & 2.863443 \\
\hline $\mathrm{Zn}$ & -5.915577 & 2.908355 & 1.990281 \\
\hline $\mathrm{Zn}$ & -6.717444 & 5.567839 & 3.706060 \\
\hline $\mathrm{O}$ & -6.418632 & 4.629274 & 5.360104 \\
\hline $\mathrm{Zn}$ & -4.757526 & 5.124429 & 6.383116 \\
\hline $\mathrm{O}$ & -4.191801 & 3.218994 & 6.628489 \\
\hline $\mathrm{Zn}$ & -2.535637 & 2.908032 & 5.697553 \\
\hline $\mathrm{O}$ & -1.179193 & 4.272133 & 5.170812 \\
\hline $\mathrm{Zn}$ & -1.907492 & 6.057065 & 5.067534 \\
\hline $\mathrm{O}$ & -3.635266 & 6.631321 & 5.923945 \\
\hline $\mathrm{Zn}$ & -4.095919 & 7.428440 & 4.153412 \\
\hline $\mathrm{O}$ & -2.243467 & 7.133962 & 3.427665 \\
\hline $\mathrm{Zn}$ & -2.199957 & 6.130390 & 1.779330 \\
\hline $\mathrm{Zn}$ & -5.305924 & 6.041304 & 1.410958 \\
\hline $\mathrm{O}$ & -5.641679 & 7.128476 & 3.048711 \\
\hline $\mathrm{Zn}$ & 0.736567 & -0.842411 & -2.830416 \\
\hline $\mathrm{Zn}$ & 0.074989 & -3.146462 & -0.600663 \\
\hline $\mathrm{O}$ & -1.777475 & -2.852015 & 0.125082 \\
\hline $\mathrm{Zn}$ & -1.820952 & -1.848330 & 1.773350 \\
\hline $\mathrm{O}$ & -2.603604 & -0.010217 & 1.783345 \\
\hline $\mathrm{Zn}$ & -1.009668 & 0.445770 & 3.130741 \\
\hline $\mathrm{Zn}$ & -0.923957 & 3.303493 & 3.454932 \\
\hline $\mathrm{O}$ & -2.231312 & 1.735056 & 4.137902 \\
\hline $\mathrm{O}$ & 2.397695 & -0.347273 & -1.807417 \\
\hline $\mathrm{Zn}$ & 2.696510 & -1.285840 & -0.153379 \\
\hline $\mathrm{O}$ & 2.672414 & -0.396219 & 1.643878 \\
\hline $\mathrm{Zn}$ & 1.285025 & -1.759270 & 2.141751 \\
\hline $\mathrm{O}$ & -0.363801 & -1.442720 & 3.088340 \\
\hline $\mathrm{O}$ & 0.170901 & 1.063030 & -3.075833 \\
\hline $\mathrm{Zn}$ & 1.614679 & 1.502351 & -1.753606 \\
\hline
\end{tabular}




$\begin{array}{crrr}\mathrm{O} & 1.507644 & 2.436153 & -0.069112 \\ \mathrm{Zn} & 1.894665 & 1.373682 & 1.562382 \\ \mathrm{O} & 0.204781 & 1.912590 & 2.469489 \\ \mathrm{Zn} & -0.276820 & 2.955992 & 0.689222 \\ \mathrm{O} & -1.417290 & 4.292274 & 1.769318 \\ \mathrm{Zn} & -3.011240 & 3.836278 & 0.421934 \\ \mathrm{O} & -3.657109 & 5.724770 & 0.464349 \\ \mathrm{Zn} & -1.485246 & 1.373985 & -2.144864 \\ \mathrm{O} & -1.789598 & 2.546994 & -0.585238 \\ \mathrm{O} & 1.620759 & -2.846476 & 0.504018 \\ \mathrm{O} & -4.225689 & 2.369455 & 1.083178 \\ \mathrm{O} & -6.693337 & 4.678252 & 1.908791 \\ \mathrm{O} & -5.528564 & 1.845875 & 3.621764 \\ \mathrm{Zn} & -5.635580 & 2.779662 & 5.306270\end{array}$

$(\mathrm{ZnO})_{36}$ in ethanol

$\begin{array}{crrr}\mathrm{O} & 0.043415 & -0.044643 & 0.053915 \\ \mathrm{Zn} & -1.722268 & -0.094815 & -0.756060 \\ \mathrm{O} & -2.353894 & -1.381980 & -2.147955 \\ \mathrm{Zn} & -4.012604 & -1.545241 & -1.034833 \\ \mathrm{O} & -3.534049 & 0.028313 & 0.097345 \\ \mathrm{Zn} & -3.526655 & -0.756190 & 1.897492 \\ \mathrm{Zn} & -2.074921 & -0.670727 & 4.737144 \\ \mathrm{O} & -1.776413 & -0.778528 & 2.795854 \\ \mathrm{Zn} & -0.110104 & -0.109699 & 2.011787 \\ \mathrm{Zn} & 1.143475 & -1.596720 & -0.448791 \\ \mathrm{O} & 0.349596 & -3.133090 & -1.353299 \\ \mathrm{Zn} & 1.304574 & -4.842583 & -1.476175 \\ \mathrm{O} & 0.341722 & -6.515872 & -1.197819 \\ \mathrm{Zn} & 1.187817 & -8.023187 & -0.285152 \\ \mathrm{O} & 2.915646 & -7.781462 & 0.623673 \\ \mathrm{Zn} & 2.763386 & -7.934129 & 2.579420 \\ \mathrm{O} & 1.565622 & -9.327918 & 3.278079 \\ \mathrm{Zn} & -0.056957 & -9.543913 & 2.195985 \\ \mathrm{O} & 0.096343 & -9.574075 & 0.239632 \\ \mathrm{Zn} & -1.658017 & -9.509359 & -0.589222 \\ \mathrm{Zn} & -3.382162 & -8.774268 & 2.052463 \\ \mathrm{O} & -4.685894 & -7.451585 & 2.637125 \\ \mathrm{Zn} & -4.937657 & -7.326594 & 4.590016 \\ \mathrm{O} & -6.463957 & -6.248603 & 5.159739 \\ \mathrm{Zn} & -5.658166 & -4.755118 & 6.243209 \\ \mathrm{O} & -3.918827 & -4.739632 & 7.114650 \\ \mathrm{Zn} & -2.829463 & -6.375704 & 7.020999\end{array}$




\begin{tabular}{|c|c|c|c|}
\hline $\mathrm{O}$ & -1.019928 & -6.406063 & 7.794414 \\
\hline $\mathrm{Zn}$ & 0.050340 & -7.941089 & 7.281176 \\
\hline $\mathrm{O}$ & -0.541756 & -9.529924 & 6.193668 \\
\hline $\mathrm{Zn}$ & -1.982361 & -8.906814 & 5.025395 \\
\hline $\mathrm{O}$ & -3.377350 & -7.705369 & 5.695875 \\
\hline $\mathrm{O}$ & 1.519027 & -0.072289 & 3.111599 \\
\hline $\mathrm{Zn}$ & 1.099675 & -0.155531 & 5.000588 \\
\hline $\mathrm{Zn}$ & -0.057149 & -1.503640 & 7.070325 \\
\hline $\mathrm{O}$ & -1.118341 & -3.038260 & 7.598678 \\
\hline $\mathrm{Zn}$ & -2.918037 & -3.085374 & 6.808015 \\
\hline $\mathrm{O}$ & -3.511396 & -1.776565 & 5.492274 \\
\hline $\mathrm{Zn}$ & -5.087527 & -2.138984 & 4.397000 \\
\hline $\mathrm{O}$ & -6.467537 & -3.342404 & 5.074142 \\
\hline $\mathrm{Zn}$ & -6.796020 & -4.835225 & 3.771930 \\
\hline $\mathrm{O}$ & -6.605685 & -4.810445 & 1.840639 \\
\hline $\mathrm{Zn}$ & -5.759734 & -3.163386 & 1.168722 \\
\hline $\mathrm{O}$ & -5.020499 & -3.148785 & -0.656054 \\
\hline $\mathrm{Zn}$ & -4.221862 & -4.826557 & -1.298195 \\
\hline $\mathrm{O}$ & -4.997337 & -6.500070 & -0.621717 \\
\hline $\mathrm{Zn}$ & -5.538244 & -6.354994 & 1.259002 \\
\hline $\mathrm{Zn}$ & -3.935186 & -8.069449 & -0.985593 \\
\hline $\mathrm{O}$ & -3.467139 & -9.567345 & 0.267869 \\
\hline $\mathrm{Zn}$ & 2.691625 & -1.497061 & 2.425377 \\
\hline $\mathrm{O}$ & 3.018364 & -3.036761 & 3.583590 \\
\hline $\mathrm{Zn}$ & 2.156933 & -3.074538 & 5.346294 \\
\hline $\mathrm{O}$ & 1.811398 & -1.450745 & 6.361573 \\
\hline $\mathrm{O}$ & 2.868560 & -1.761763 & 0.475888 \\
\hline $\mathrm{Zn}$ & 3.766557 & -3.419900 & 0.045631 \\
\hline $\mathrm{Zn}$ & 3.800479 & -6.141506 & 0.077680 \\
\hline $\mathrm{O}$ & 3.251917 & -4.805867 & -1.303851 \\
\hline $\mathrm{O}$ & 4.697920 & -4.751933 & 1.215818 \\
\hline $\mathrm{Zn}$ & 3.805321 & -4.721952 & 2.958018 \\
\hline $\mathrm{O}$ & 3.132881 & -6.394012 & 3.717775 \\
\hline $\mathrm{Zn}$ & 2.245805 & -6.349238 & 5.468094 \\
\hline $\mathrm{Zn}$ & 1.134848 & -9.258306 & 5.159580 \\
\hline $\mathrm{O}$ & 1.894699 & -7.959534 & 6.501772 \\
\hline $\mathrm{O}$ & 1.217865 & -4.723641 & 5.865612 \\
\hline $\mathrm{Zn}$ & -0.162933 & -4.714110 & 7.241004 \\
\hline $\mathrm{O}$ & -0.679784 & 0.018418 & 5.917632 \\
\hline $\mathrm{O}$ & -1.733373 & -9.015602 & 3.076846 \\
\hline $\mathrm{O}$ & -5.040958 & -1.860280 & 2.452145 \\
\hline $\mathrm{Zn}$ & -1.480198 & -3.130745 & -2.081598 \\
\hline $\mathrm{O}$ & -2.426158 & -4.847545 & -2.115212 \\
\hline $\mathrm{Zn}$ & -1.456899 & -6.542457 & -2.006679 \\
\hline $\mathrm{O}$ & -2.287267 & -8.318937 & -2.076409 \\
\hline
\end{tabular}


$(\mathrm{ZnO})_{36}$ in 1-propanol

\begin{tabular}{|c|c|c|c|}
\hline $\mathrm{O}$ & -0.016786 & -0.026340 & 0.040086 \\
\hline $\mathrm{Zn}$ & 1.797864 & 0.001799 & -0.727310 \\
\hline $\mathrm{O}$ & 2.016431 & 0.219826 & -2.671739 \\
\hline $\mathrm{Zn}$ & 0.739999 & 1.596729 & -3.245408 \\
\hline $\mathrm{Zn}$ & 3.815419 & 0.708148 & -3.195266 \\
\hline $\mathrm{O}$ & 4.548136 & 2.023639 & -4.506632 \\
\hline $\mathrm{Zn}$ & 6.016527 & 2.309124 & -3.164862 \\
\hline $\mathrm{O}$ & 5.478718 & 0.719782 & -2.077024 \\
\hline $\mathrm{Zn}$ & 4.949419 & 1.244469 & -0.275353 \\
\hline $\mathrm{Zn}$ & 3.077995 & 0.819584 & 2.402069 \\
\hline $\mathrm{O}$ & 4.088813 & 2.239411 & 3.296728 \\
\hline $\mathrm{Zn}$ & 5.782481 & 2.853165 & 2.513390 \\
\hline $\mathrm{O}$ & 6.946142 & 4.027603 & 3.548418 \\
\hline $\mathrm{Zn}$ & 5.782110 & 5.337908 & 4.534806 \\
\hline $\mathrm{O}$ & 6.548599 & 6.928784 & 3.574671 \\
\hline $\mathrm{Zn}$ & 7.344740 & 5.571773 & 2.335589 \\
\hline $\mathrm{Zn}$ & 6.529797 & 7.115015 & -0.424251 \\
\hline $\mathrm{Zn}$ & 5.241264 & 8.820430 & -2.785053 \\
\hline $\mathrm{O}$ & 3.736872 & 8.749734 & -4.119610 \\
\hline $\mathrm{Zn}$ & 3.032056 & 6.931012 & -4.142158 \\
\hline $\mathrm{O}$ & 4.236173 & 5.384217 & -4.213226 \\
\hline $\mathrm{Zn}$ & 3.427177 & 3.597471 & -4.252385 \\
\hline $\mathrm{O}$ & 1.543749 & 3.312147 & -3.797489 \\
\hline $\mathrm{Zn}$ & 0.452385 & 4.899619 & -4.103569 \\
\hline $\mathrm{O}$ & -1.460217 & 4.679696 & -4.445148 \\
\hline $\mathrm{Zn}$ & -2.448614 & 5.918837 & -3.210579 \\
\hline $\mathrm{O}$ & -3.320213 & 4.418017 & -2.202729 \\
\hline $\mathrm{Zn}$ & -2.118499 & 3.206878 & -3.253720 \\
\hline $\mathrm{O}$ & -1.157101 & 1.590269 & -2.765106 \\
\hline $\mathrm{Zn}$ & -1.106215 & 1.376975 & -0.804689 \\
\hline $\mathrm{O}$ & -1.489072 & 2.973630 & 0.261488 \\
\hline $\mathrm{Zn}$ & -2.606098 & 4.450772 & -0.383180 \\
\hline $\mathrm{O}$ & -2.482143 & 6.173306 & 0.539333 \\
\hline $\mathrm{Zn}$ & -2.019353 & 6.144246 & 2.431925 \\
\hline $\mathrm{O}$ & -2.038567 & 7.800603 & 3.470047 \\
\hline $\mathrm{Zn}$ & -0.298868 & 7.869409 & 4.454997 \\
\hline $\mathrm{Zn}$ & 1.989950 & 9.129710 & 2.707872 \\
\hline $\mathrm{Zn}$ & 3.873913 & 9.274421 & 0.003446 \\
\hline $\mathrm{O}$ & 5.131318 & 7.937762 & 0.680090 \\
\hline $\mathrm{Zn}$ & 5.061816 & 7.774263 & 2.628788 \\
\hline $\mathrm{O}$ & 3.400190 & 8.099393 & 3.597424 \\
\hline
\end{tabular}




\begin{tabular}{|c|c|c|c|}
\hline $\mathrm{Zn}$ & 2.778112 & 6.681813 & 4.798090 \\
\hline $\mathrm{O}$ & 3.946696 & 5.138131 & 5.118074 \\
\hline $\mathrm{Zn}$ & 3.245897 & 3.364076 & 4.657756 \\
\hline $\mathrm{O}$ & 1.324642 & 3.074119 & 5.014433 \\
\hline $\mathrm{Zn}$ & 0.283891 & 4.680800 & 4.616408 \\
\hline $\mathrm{O}$ & -1.080036 & 4.606352 & 3.207655 \\
\hline $\mathrm{Zn}$ & -1.347648 & 2.946929 & 2.212285 \\
\hline $\mathrm{O}$ & -1.052098 & 1.271115 & 3.161579 \\
\hline $\mathrm{Zn}$ & 0.615657 & 1.445673 & 4.251050 \\
\hline $\mathrm{O}$ & 1.556222 & -0.026062 & 3.275171 \\
\hline $\mathrm{Zn}$ & 0.039004 & 0.075535 & 1.969708 \\
\hline $\mathrm{Zn}$ & -2.096855 & 7.769326 & -0.527366 \\
\hline $\mathrm{Zn}$ & 0.041674 & 8.103088 & -2.959888 \\
\hline $\mathrm{O}$ & 1.196208 & 6.624534 & -3.533823 \\
\hline $\mathrm{O}$ & -1.288020 & 9.320612 & 0.362044 \\
\hline $\mathrm{Zn}$ & 0.500810 & 9.699995 & -0.353409 \\
\hline $\mathrm{Zn}$ & 2.707073 & 9.829717 & -2.786545 \\
\hline $\mathrm{O}$ & 4.317418 & 10.194345 & -1.661580 \\
\hline $\mathrm{Zn}$ & -1.230997 & 9.211429 & 2.293270 \\
\hline $\mathrm{O}$ & 0.288626 & 9.568707 & 3.556562 \\
\hline $\mathrm{O}$ & -1.787030 & 7.584617 & -2.466909 \\
\hline $\mathrm{O}$ & 0.847785 & 6.480338 & 5.159902 \\
\hline $\mathrm{O}$ & 2.064985 & 9.286208 & 0.752257 \\
\hline $\mathrm{O}$ & 0.837880 & 9.781181 & -2.302205 \\
\hline $\mathrm{O}$ & 7.505235 & 5.631336 & 0.409877 \\
\hline $\mathrm{Zn}$ & 6.936669 & 3.993659 & -0.521788 \\
\hline $\mathrm{O}$ & 5.912899 & 2.722733 & 0.565058 \\
\hline $\mathrm{O}$ & 6.725545 & 3.976846 & -2.473520 \\
\hline $\mathrm{Zn}$ & 5.885229 & 5.604426 & -3.192140 \\
\hline $\mathrm{O}$ & 6.392636 & 7.318784 & -2.375301 \\
\hline $\mathrm{O}$ & 3.220158 & 0.668753 & 0.449684 \\
\hline
\end{tabular}

$(\mathrm{ZnO})_{36}$ in 1-butanol

$\begin{array}{crrr}\mathrm{O} & -0.010422 & -0.237063 & -0.003029 \\ \mathrm{Zn} & 1.795816 & -0.024620 & -0.750897 \\ \mathrm{O} & 2.118093 & 0.137209 & -2.694638 \\ \mathrm{Zn} & 3.924579 & 0.641427 & -3.172346 \\ \mathrm{O} & 4.652862 & 2.002291 & -4.430849 \\ \mathrm{Zn} & 5.975727 & 2.397233 & -2.961588 \\ \mathrm{Zn} & 4.948722 & 1.304756 & -0.122237 \\ \mathrm{Zn} & 2.947137 & 0.866442 & 2.382094 \\ \mathrm{O} & 3.832542 & 2.325104 & 3.342198 \\ \mathrm{Zn} & 5.515201 & 3.054475 & 2.652831\end{array}$




\begin{tabular}{|c|c|c|c|}
\hline $\mathrm{O}$ & 6.468789 & 4.399211 & 3.698828 \\
\hline $\mathrm{Zn}$ & 5.171811 & 5.656808 & 4.595103 \\
\hline $\mathrm{O}$ & 5.805513 & 7.250361 & 3.570215 \\
\hline $\mathrm{Zn}$ & 6.841919 & 5.928531 & 2.473961 \\
\hline $\mathrm{O}$ & 7.049162 & 5.925808 & 0.547917 \\
\hline $\mathrm{Zn}$ & 6.195060 & 7.396176 & -0.432209 \\
\hline $\mathrm{Zn}$ & 4.873852 & 8.809225 & -3.071773 \\
\hline $\mathrm{Zn}$ & 2.730676 & 6.761828 & -4.435677 \\
\hline $\mathrm{O}$ & 3.970265 & 5.284762 & -4.080883 \\
\hline $\mathrm{Zn}$ & 3.368564 & 3.466273 & -4.477787 \\
\hline $\mathrm{O}$ & 1.446814 & 3.105820 & -4.249163 \\
\hline $\mathrm{Zn}$ & 0.830615 & 1.432797 & -3.425045 \\
\hline $\mathrm{O}$ & -0.995879 & 1.451440 & -2.713275 \\
\hline $\mathrm{Zn}$ & -1.185848 & 1.133662 & -0.777674 \\
\hline $\mathrm{O}$ & -1.997669 & 2.535839 & 0.316123 \\
\hline $\mathrm{Zn}$ & -2.803458 & 4.064240 & -0.584467 \\
\hline $\mathrm{Zn}$ & -2.569339 & 5.842069 & 2.169089 \\
\hline $\mathrm{O}$ & -2.562762 & 7.592420 & 3.047901 \\
\hline $\mathrm{Zn}$ & -0.896772 & 7.790263 & 4.130878 \\
\hline $\mathrm{Zn}$ & 1.324825 & 8.986699 & 2.242171 \\
\hline $\mathrm{Zn}$ & 4.391779 & 7.936384 & 2.400418 \\
\hline $\mathrm{O}$ & 4.883334 & 8.452552 & 0.580666 \\
\hline $\mathrm{Zn}$ & 3.515977 & 9.562807 & -0.282548 \\
\hline $\mathrm{O}$ & 3.827175 & 10.200445 & -2.096719 \\
\hline $\mathrm{Zn}$ & 2.280603 & 9.645060 & -3.239673 \\
\hline $\mathrm{Zn}$ & -0.391166 & 7.836436 & -3.409740 \\
\hline $\mathrm{Zn}$ & -2.635977 & 5.520088 & -3.550107 \\
\hline $\mathrm{Zn}$ & 0.271654 & 4.635211 & -4.565716 \\
\hline $\mathrm{O}$ & -1.653015 & 4.290536 & -4.801185 \\
\hline $\mathrm{Zn}$ & -2.095576 & 2.850421 & -3.475738 \\
\hline $\mathrm{O}$ & -3.363357 & 4.011883 & -2.443148 \\
\hline $\mathrm{Zn}$ & -0.048115 & -0.130150 & 1.924853 \\
\hline $\mathrm{Zn}$ & -1.831177 & 2.561594 & 2.270048 \\
\hline $\mathrm{O}$ & -1.776077 & 4.327831 & 3.116692 \\
\hline $\mathrm{Zn}$ & -0.335791 & 4.594397 & 4.428540 \\
\hline $\mathrm{Zn}$ & 2.087123 & 6.511103 & 4.491801 \\
\hline $\mathrm{O}$ & 2.530085 & 7.579821 & 2.908111 \\
\hline $\mathrm{O}$ & 1.467779 & -0.077020 & 3.243111 \\
\hline $\mathrm{Zn}$ & 0.361498 & 1.298389 & 4.193456 \\
\hline $\mathrm{Zn}$ & 2.820767 & 3.390885 & 4.626700 \\
\hline $\mathrm{O}$ & 3.375958 & 5.196652 & 5.154179 \\
\hline $\mathrm{O}$ & -1.280522 & 0.896808 & 3.129333 \\
\hline $\mathrm{O}$ & 0.874600 & 3.082910 & 4.740563 \\
\hline $\mathrm{O}$ & -2.752673 & 5.856702 & 0.217433 \\
\hline $\mathrm{Zn}$ & -2.391329 & 7.400068 & -0.929485 \\
\hline
\end{tabular}




$\begin{array}{crrr}\mathrm{O} & -2.200205 & 7.287033 & -2.889112 \\ \mathrm{O} & -1.549339 & 8.955340 & -0.079265 \\ \mathrm{Zn} & 0.168431 & 9.563526 & -0.833130 \\ \mathrm{O} & 0.408172 & 9.526348 & -2.781618 \\ \mathrm{Zn} & -1.757849 & 8.969522 & 1.843587 \\ \mathrm{O} & -0.325026 & 9.450515 & 3.173726 \\ \mathrm{O} & 0.184037 & 6.411599 & 4.953138 \\ \mathrm{O} & 1.687099 & 9.564110 & 0.412550 \\ \mathrm{O} & 3.440997 & 8.575538 & -4.458649 \\ \mathrm{Zn} & 6.687744 & 4.219930 & -0.348893 \\ \mathrm{O} & 5.884886 & 2.791993 & 0.737088 \\ \mathrm{O} & 6.562486 & 4.116303 & -2.299351 \\ \mathrm{Zn} & 5.610936 & 5.643864 & -3.086564 \\ \mathrm{O} & 6.049540 & 7.426870 & -2.389528 \\ \mathrm{O} & 0.821162 & 6.448475 & -4.073876 \\ \mathrm{O} & 5.488054 & 0.764975 & -1.922283 \\ \mathrm{O} & 3.145741 & 0.773479 & 0.431371\end{array}$

$(\mathrm{ZnO})_{36}$ in 1-pentanol

$\begin{array}{crrr}\mathrm{O} & 0.075638 & -0.126734 & -0.001407 \\ \mathrm{Zn} & 1.876410 & -0.078017 & -0.791495 \\ \mathrm{O} & 2.108070 & 0.067164 & -2.742494 \\ \mathrm{Zn} & 3.911621 & 0.559131 & -3.244271 \\ \mathrm{O} & 4.619113 & 1.911067 & -4.521198 \\ \mathrm{Zn} & 5.971831 & 2.314299 & -3.089436 \\ \mathrm{Zn} & 4.991019 & 1.219346 & -0.223459 \\ \mathrm{O} & 3.254179 & 0.603834 & 0.430304 \\ \mathrm{Zn} & 2.966379 & 0.853196 & 2.362499 \\ \mathrm{O} & 3.845704 & 2.347508 & 3.264620 \\ \mathrm{Zn} & 5.550679 & 2.987902 & 2.540880 \\ \mathrm{O} & 6.512138 & 4.323928 & 3.591929 \\ \mathrm{Zn} & 5.230710 & 5.597303 & 4.484514 \\ \mathrm{O} & 5.877466 & 7.171386 & 3.440819 \\ \mathrm{Zn} & 6.902635 & 5.839176 & 2.354571 \\ \mathrm{O} & 7.083294 & 5.838466 & 0.428194 \\ \mathrm{Zn} & 6.224458 & 7.308550 & -0.546330 \\ \mathrm{Zn} & 4.855280 & 8.693995 & -3.161201 \\ \mathrm{Zn} & 2.690436 & 6.655887 & -4.481423 \\ \mathrm{O} & 3.957951 & 5.190385 & -4.179748 \\ \mathrm{Zn} & 3.331835 & 3.372931 & -4.549902 \\ \mathrm{O} & 1.413931 & 3.012556 & -4.288863 \\ \mathrm{Zn} & 0.791203 & 1.339582 & -3.465063 \\ \mathrm{O} & -1.036668 & 1.366245 & -2.752152 \\ & & & \end{array}$




\begin{tabular}{|c|c|c|c|}
\hline $\mathrm{Zn}$ & -1.146704 & 1.188445 & -0.801127 \\
\hline $\mathrm{Zn}$ & -2.141373 & 2.783035 & -3.481322 \\
\hline $\mathrm{Zn}$ & 0.231221 & 4.537596 & -4.594698 \\
\hline $\mathrm{O}$ & -1.700000 & 4.219684 & -4.813340 \\
\hline $\mathrm{Zn}$ & -2.606911 & 5.470173 & -3.528097 \\
\hline $\mathrm{O}$ & -3.365123 & 3.970949 & -2.433856 \\
\hline $\mathrm{Zn}$ & -2.776716 & 4.113954 & -0.588091 \\
\hline $\mathrm{Zn}$ & -2.530803 & 5.894376 & 2.154880 \\
\hline $\mathrm{O}$ & -2.519481 & 7.657388 & 3.006624 \\
\hline $\mathrm{Zn}$ & -0.832703 & 7.843472 & 4.062119 \\
\hline $\mathrm{Zn}$ & 1.413807 & 9.020082 & 2.158199 \\
\hline $\mathrm{Zn}$ & 4.454728 & 7.852139 & 2.285307 \\
\hline $\mathrm{O}$ & 4.904270 & 8.362772 & 0.455351 \\
\hline $\mathrm{Zn}$ & 3.521377 & 9.467171 & -0.395624 \\
\hline $\mathrm{O}$ & 3.916148 & 10.160501 & -2.179596 \\
\hline $\mathrm{Zn}$ & 2.320674 & 9.666153 & -3.277561 \\
\hline $\mathrm{Zn}$ & -0.359827 & 7.784149 & -3.426265 \\
\hline $\mathrm{O}$ & -2.162812 & 7.238960 & -2.885545 \\
\hline $\mathrm{Zn}$ & -2.371544 & 7.458274 & -0.930429 \\
\hline $\mathrm{Zn}$ & -1.750459 & 9.045027 & 1.794055 \\
\hline $\mathrm{O}$ & -0.247058 & 9.472333 & 3.067826 \\
\hline $\mathrm{Zn}$ & -0.034510 & -0.094464 & 1.922585 \\
\hline $\mathrm{Zn}$ & -1.793449 & 2.605913 & 2.250527 \\
\hline $\mathrm{O}$ & -1.736462 & 4.375558 & 3.091546 \\
\hline $\mathrm{Zn}$ & -0.282810 & 4.644648 & 4.387020 \\
\hline $\mathrm{Zn}$ & 2.151024 & 6.554548 & 4.445977 \\
\hline $\mathrm{O}$ & 2.606079 & 7.634732 & 2.873646 \\
\hline $\mathrm{O}$ & 1.480971 & -0.049825 & 3.242635 \\
\hline $\mathrm{Zn}$ & 0.388774 & 1.340835 & 4.179376 \\
\hline $\mathrm{Zn}$ & 2.863385 & 3.419567 & 4.567631 \\
\hline $\mathrm{O}$ & 3.431436 & 5.222746 & 5.091403 \\
\hline $\mathrm{O}$ & -1.259306 & 0.943150 & 3.122658 \\
\hline $\mathrm{O}$ & 0.914173 & 3.125968 & 4.708114 \\
\hline $\mathrm{O}$ & -1.953398 & 2.587813 & 0.297514 \\
\hline $\mathrm{O}$ & -2.721242 & 5.907069 & 0.204430 \\
\hline $\mathrm{O}$ & -1.636128 & 9.097128 & -0.137503 \\
\hline $\mathrm{Zn}$ & 0.098033 & 9.660447 & -0.882614 \\
\hline $\mathrm{O}$ & 0.449920 & 9.471175 & -2.820141 \\
\hline $\mathrm{O}$ & 0.242654 & 6.464329 & 4.893188 \\
\hline $\mathrm{O}$ & 1.677902 & 9.443327 & 0.260516 \\
\hline $\mathrm{O}$ & 3.380891 & 8.472501 & -4.497613 \\
\hline $\mathrm{Zn}$ & 6.713450 & 4.135339 & -0.468694 \\
\hline $\mathrm{O}$ & 5.910875 & 2.718589 & 0.628596 \\
\hline $\mathrm{O}$ & 6.569175 & 4.025095 & -2.416845 \\
\hline $\mathrm{Zn}$ & 5.608783 & 5.546128 & -3.204009 \\
\hline
\end{tabular}




$$
\begin{aligned}
& \mathrm{O} \\
& \mathrm{O} \\
& \mathrm{O}
\end{aligned}
$$

6.051488

0.792259

5.503502
7.323094

6.343202

0.690668
$-2.500716$

$-4.093378$

\begin{tabular}{|c|c|c|c|}
\hline $\mathrm{O}$ & -0.096949 & -0.205523 & 0.084824 \\
\hline $\mathrm{Zn}$ & 1.668607 & -0.246779 & -0.778842 \\
\hline $\mathrm{O}$ & 1.704078 & -0.055028 & -2.728792 \\
\hline $\mathrm{Zn}$ & 3.477460 & -0.082038 & -3.488602 \\
\hline $\mathrm{O}$ & 4.123588 & 1.099786 & -4.963183 \\
\hline $\mathrm{Zn}$ & 5.702218 & 1.425976 & -3.800649 \\
\hline $\mathrm{Zn}$ & 4.962235 & 0.540762 & -0.746656 \\
\hline $\mathrm{O}$ & 3.310678 & 0.095773 & 0.211411 \\
\hline $\mathrm{Zn}$ & 3.241804 & 0.516421 & 2.112378 \\
\hline $\mathrm{O}$ & 4.340061 & 1.929352 & 2.913021 \\
\hline $\mathrm{Zn}$ & 5.959410 & 2.360643 & 1.897960 \\
\hline $\mathrm{O}$ & 7.226735 & 3.668952 & 2.603348 \\
\hline $\mathrm{Zn}$ & 6.143259 & 5.096294 & 3.483694 \\
\hline $\mathrm{O}$ & 6.915520 & 6.554491 & 2.356890 \\
\hline $\mathrm{Zn}$ & 7.669096 & 5.069200 & 1.242081 \\
\hline $\mathrm{Zn}$ & 6.587730 & 6.477487 & -1.516191 \\
\hline $\mathrm{O}$ & 5.567825 & 7.759303 & -0.443953 \\
\hline $\mathrm{Zn}$ & 5.508568 & 7.595312 & 1.502352 \\
\hline $\mathrm{O}$ & 3.864879 & 7.979588 & 2.483753 \\
\hline $\mathrm{Zn}$ & 2.466503 & 8.958520 & 1.536410 \\
\hline $\mathrm{Zn}$ & 4.164827 & 8.835521 & -1.270240 \\
\hline $\mathrm{Zn}$ & 5.132099 & 7.922225 & -4.157951 \\
\hline $\mathrm{O}$ & 3.605492 & 7.816529 & -5.445585 \\
\hline $\mathrm{Zn}$ & 2.780070 & 6.042274 & -5.434654 \\
\hline $\mathrm{O}$ & 3.902093 & 4.475280 & -5.147782 \\
\hline $\mathrm{Zn}$ & 3.052095 & 2.706568 & -5.165292 \\
\hline $\mathrm{O}$ & 1.141356 & 2.637417 & -4.744066 \\
\hline $\mathrm{Zn}$ & 0.477712 & 1.229157 & -3.566459 \\
\hline $\mathrm{O}$ & -1.298325 & 1.307710 & -2.737358 \\
\hline $\mathrm{Zn}$ & -2.390013 & 2.709494 & -3.510868 \\
\hline $\mathrm{O}$ & -3.414865 & 4.025752 & -2.414574 \\
\hline $\mathrm{Zn}$ & -2.664463 & 5.415608 & -3.629591 \\
\hline $\mathrm{O}$ & -1.903094 & 4.056154 & -4.897276 \\
\hline $\mathrm{Zn}$ & 0.035633 & 4.211000 & -5.060222 \\
\hline $\mathrm{O}$ & 0.860554 & 5.992383 & -5.043285 \\
\hline $\mathrm{Zn}$ & -0.128949 & 7.436622 & -4.173396 \\
\hline $\mathrm{O}$ & -1.784474 & 7.074565 & -3.191156 \\
\hline $\mathrm{Zn}$ & -1.777378 & 7.478453 & -1.277264 \\
\hline
\end{tabular}

$-2.029570$

$(\mathrm{ZnO})_{36}$ in 1-octanol 


\begin{tabular}{|c|c|c|c|}
\hline $\mathrm{Zn}$ & 0.796969 & 9.184114 & -1.485494 \\
\hline $\mathrm{Zn}$ & 2.658819 & 9.019764 & -4.162352 \\
\hline $\mathrm{O}$ & 4.311191 & 9.421143 & -3.116564 \\
\hline $\mathrm{Zn}$ & 0.003186 & -0.085817 & 2.013534 \\
\hline $\mathrm{Zn}$ & -1.454687 & 2.757633 & 2.158993 \\
\hline $\mathrm{O}$ & -0.878004 & 4.534131 & 2.746661 \\
\hline $\mathrm{Zn}$ & -1.658341 & 6.066659 & 1.820851 \\
\hline $\mathrm{O}$ & -1.592505 & 7.841236 & 2.631997 \\
\hline $\mathrm{Zn}$ & 0.174769 & 7.968659 & 3.561514 \\
\hline $\mathrm{O}$ & 1.317479 & 6.532697 & 4.166780 \\
\hline $\mathrm{Zn}$ & 3.219126 & 6.622111 & 3.735731 \\
\hline $\mathrm{O}$ & 4.314510 & 5.047168 & 4.087563 \\
\hline $\mathrm{Zn}$ & 3.474352 & 3.270763 & 4.048414 \\
\hline $\mathrm{O}$ & 1.606331 & 3.167094 & 4.614821 \\
\hline $\mathrm{Zn}$ & 0.545387 & 4.731731 & 4.091326 \\
\hline $\mathrm{Zn}$ & 0.813014 & 1.475981 & 4.095089 \\
\hline $\mathrm{O}$ & -0.958266 & 1.201290 & 3.207066 \\
\hline $\mathrm{Zn}$ & -1.268665 & 1.104120 & -0.790515 \\
\hline $\mathrm{O}$ & -2.033784 & 2.542377 & 0.307541 \\
\hline $\mathrm{Zn}$ & -2.616780 & 4.148853 & -0.636843 \\
\hline $\mathrm{O}$ & -2.064362 & 5.946611 & -0.090612 \\
\hline $\mathrm{O}$ & 1.668485 & -0.033061 & 3.120507 \\
\hline $\mathrm{O}$ & -0.967317 & 9.131615 & -0.604333 \\
\hline $\mathrm{Zn}$ & -0.809006 & 9.139366 & 1.320200 \\
\hline $\mathrm{O}$ & 0.808429 & 9.493119 & 2.421461 \\
\hline $\mathrm{O}$ & 2.392463 & 8.841985 & -0.419420 \\
\hline $\mathrm{O}$ & 0.871724 & 8.968558 & -3.436746 \\
\hline $\mathrm{O}$ & 7.528070 & 4.977919 & -0.682254 \\
\hline $\mathrm{Zn}$ & 6.790323 & 3.271476 & -1.317517 \\
\hline $\mathrm{O}$ & 6.078275 & 1.983947 & -0.024691 \\
\hline $\mathrm{O}$ & 6.318988 & 3.157115 & -3.206883 \\
\hline $\mathrm{Zn}$ & 5.531604 & 4.709413 & -4.106384 \\
\hline $\mathrm{O}$ & 6.126599 & 6.441542 & -3.415609 \\
\hline $\mathrm{O}$ & 5.248435 & -0.083925 & -2.564480 \\
\hline
\end{tabular}

$(\mathrm{ZnO})_{36}-(\mathrm{ZnO})_{36}$ in ethanol

$\begin{array}{crrr}\mathrm{O} & -0.063286 & -0.152303 & 0.112371 \\ \mathrm{Zn} & 1.717107 & -0.192380 & -0.737643 \\ \mathrm{O} & 3.263037 & 0.133463 & 0.419434 \\ \mathrm{Zn} & 5.076991 & 0.141985 & -0.304137 \\ \mathrm{O} & 5.573782 & -0.390030 & -2.110181 \\ \mathrm{Zn} & 6.416724 & 1.152320 & -3.074553 \\ \mathrm{Zn} & 6.943845 & 4.373051 & -2.819934\end{array}$




\begin{tabular}{|c|c|c|c|}
\hline $\mathrm{O}$ & 7.753538 & 5.881213 & -1.837643 \\
\hline $\mathrm{Zn}$ & 9.711260 & 6.770863 & -1.611156 \\
\hline $\mathrm{Zn}$ & 12.379887 & 4.897206 & -1.760465 \\
\hline $\mathrm{O}$ & 14.034131 & 4.366075 & -2.687261 \\
\hline $\mathrm{Zn}$ & 15.498071 & 4.304670 & -1.327468 \\
\hline $\mathrm{O}$ & 16.624876 & 5.643183 & -2.327979 \\
\hline $\mathrm{Zn}$ & 16.985911 & 7.167951 & -1.178738 \\
\hline $\mathrm{O}$ & 16.468635 & 8.980947 & -1.703853 \\
\hline $\mathrm{Zn}$ & 17.315849 & 10.450590 & -0.731471 \\
\hline $\mathrm{O}$ & 17.381583 & 12.220837 & -1.557797 \\
\hline $\mathrm{Zn}$ & 16.554683 & 13.500101 & -0.237911 \\
\hline $\mathrm{Zn}$ & 13.489111 & 13.111217 & -0.557997 \\
\hline $\mathrm{O}$ & 12.296345 & 11.941952 & -1.581844 \\
\hline $\mathrm{Zn}$ & 10.493029 & 11.953250 & -0.801822 \\
\hline $\mathrm{O}$ & 10.350531 & 12.055726 & 1.143400 \\
\hline $\mathrm{Zn}$ & 11.582945 & 13.229007 & 2.120593 \\
\hline $\mathrm{O}$ & 11.078982 & 13.762272 & 3.922494 \\
\hline $\mathrm{Zn}$ & 12.718271 & 13.312744 & 4.985103 \\
\hline $\mathrm{O}$ & 11.717273 & 12.065953 & 6.215525 \\
\hline $\mathrm{Zn}$ & 12.459719 & 10.262173 & 6.102566 \\
\hline $\mathrm{O}$ & 11.292214 & 8.737047 & 5.735682 \\
\hline $\mathrm{Zn}$ & 12.118864 & 6.957290 & 5.752381 \\
\hline $\mathrm{Zn}$ & 9.532720 & 5.651063 & 4.238190 \\
\hline $\mathrm{O}$ & 10.411186 & 4.321786 & 2.841549 \\
\hline $\mathrm{Zn}$ & 8.584008 & 3.421311 & 2.726647 \\
\hline $\mathrm{Zn}$ & 6.186283 & 1.375283 & 2.624034 \\
\hline $\mathrm{O}$ & 4.379263 & 1.398470 & 3.394518 \\
\hline $\mathrm{Zn}$ & 3.817747 & 2.279379 & 5.073622 \\
\hline $\mathrm{O}$ & 1.906495 & 2.219512 & 5.510715 \\
\hline $\mathrm{Zn}$ & 1.388692 & 4.056369 & 5.033893 \\
\hline $\mathrm{O}$ & 0.192238 & 4.374942 & 3.519767 \\
\hline $\mathrm{Zn}$ & -0.324878 & 6.188381 & 2.995903 \\
\hline $\mathrm{O}$ & 0.035564 & 7.713385 & 4.145012 \\
\hline $\mathrm{Zn}$ & 1.159929 & 9.055225 & 3.146655 \\
\hline $\mathrm{Zn}$ & 3.019063 & 9.248574 & 0.469183 \\
\hline $\mathrm{O}$ & 2.977912 & 9.098836 & -1.483112 \\
\hline $\mathrm{Zn}$ & 4.732494 & 9.072234 & -2.310562 \\
\hline $\mathrm{O}$ & 5.628866 & 8.010644 & -3.733971 \\
\hline $\mathrm{Zn}$ & 7.124286 & 7.713035 & -2.421036 \\
\hline $\mathrm{O}$ & 8.965521 & 8.538815 & -2.207801 \\
\hline $\mathrm{Zn}$ & 9.923405 & 10.087530 & -3.006473 \\
\hline $\mathrm{O}$ & 8.962256 & 11.441211 & -1.904313 \\
\hline $\mathrm{Zn}$ & 8.077701 & 9.939841 & -0.907651 \\
\hline $\mathrm{O}$ & 8.194528 & 9.586496 & 1.097285 \\
\hline $\mathrm{Zn}$ & 8.803629 & 7.753288 & 1.650427 \\
\hline
\end{tabular}




\begin{tabular}{|c|c|c|c|}
\hline $\mathrm{O}$ & 9.811104 & 6.536862 & 0.410972 \\
\hline $\mathrm{Zn}$ & 10.401225 & 4.641125 & 0.856445 \\
\hline $\mathrm{O}$ & 12.158670 & 4.188015 & 0.056608 \\
\hline $\mathrm{Zn}$ & 13.633079 & 4.117526 & 1.348811 \\
\hline $\mathrm{O}$ & 15.468659 & 4.138739 & 0.600825 \\
\hline $\mathrm{Zn}$ & 16.585973 & 5.369678 & 1.639840 \\
\hline $\mathrm{O}$ & 16.722509 & 5.460719 & 3.598832 \\
\hline $\mathrm{Zn}$ & 17.711152 & 6.923645 & 4.403564 \\
\hline $\mathrm{O}$ & 18.802568 & 8.346933 & 3.515598 \\
\hline $\mathrm{Zn}$ & 17.958072 & 8.473786 & 1.748702 \\
\hline $\mathrm{O}$ & 17.392701 & 6.910333 & 0.725940 \\
\hline $\mathrm{Zn}$ & 0.102838 & -0.139497 & 2.048002 \\
\hline $\mathrm{O}$ & -0.723310 & 1.136599 & 3.371588 \\
\hline $\mathrm{Zn}$ & -0.656663 & 2.907137 & 2.546082 \\
\hline $\mathrm{O}$ & -0.758635 & 3.073530 & 0.598784 \\
\hline $\mathrm{Zn}$ & -1.297841 & 4.886844 & 0.066952 \\
\hline $\mathrm{O}$ & -2.146123 & 5.016959 & -1.697500 \\
\hline $\mathrm{Zn}$ & -1.056836 & 6.442563 & -2.584309 \\
\hline $\mathrm{O}$ & -0.070944 & 7.907146 & -1.779342 \\
\hline $\mathrm{Zn}$ & 0.068534 & 7.993663 & 0.179764 \\
\hline $\mathrm{O}$ & -0.732287 & 6.448801 & 1.091719 \\
\hline $\mathrm{Zn}$ & -0.711858 & 1.556727 & -0.647019 \\
\hline $\mathrm{Zn}$ & 1.201262 & 1.731277 & -3.254815 \\
\hline $\mathrm{O}$ & 2.233696 & 3.182533 & -4.066409 \\
\hline $\mathrm{Zn}$ & 4.187849 & 3.110398 & -4.295799 \\
\hline $\mathrm{O}$ & 5.352761 & 4.636845 & -3.926646 \\
\hline $\mathrm{Zn}$ & 4.526468 & 6.417734 & -3.942988 \\
\hline $\mathrm{O}$ & 2.593265 & 6.535629 & -3.579245 \\
\hline $\mathrm{Zn}$ & 1.759399 & 7.925329 & -2.487729 \\
\hline $\mathrm{Zn}$ & 3.169957 & 0.248579 & 2.368645 \\
\hline $\mathrm{Zn}$ & 0.940836 & 0.906084 & 4.450827 \\
\hline $\mathrm{O}$ & 1.640180 & -0.560294 & 3.269599 \\
\hline $\mathrm{O}$ & 2.493493 & 5.581051 & 5.586714 \\
\hline $\mathrm{Zn}$ & 4.464550 & 5.456291 & 5.318169 \\
\hline $\mathrm{O}$ & 5.079679 & 3.629772 & 5.752875 \\
\hline $\mathrm{Zn}$ & 6.739376 & 3.267516 & 4.822779 \\
\hline $\mathrm{O}$ & 7.708070 & 1.916502 & 3.726892 \\
\hline $\mathrm{Zn}$ & 1.752849 & 7.302597 & 5.118043 \\
\hline $\mathrm{O}$ & 2.624419 & 8.993513 & 4.505744 \\
\hline $\mathrm{Zn}$ & 4.278496 & 8.463716 & 3.577354 \\
\hline $\mathrm{O}$ & 5.275545 & 6.984365 & 4.402181 \\
\hline $\mathrm{Zn}$ & 6.945620 & 6.588999 & 3.430071 \\
\hline $\mathrm{O}$ & 6.838035 & 6.831336 & 1.407700 \\
\hline $\mathrm{Zn}$ & 7.845857 & 5.615981 & 0.168598 \\
\hline $\mathrm{O}$ & 8.452350 & 3.782747 & 0.723323 \\
\hline
\end{tabular}




\begin{tabular}{|c|c|c|c|}
\hline $\mathrm{Zn}$ & 7.584351 & 2.380192 & -0.342194 \\
\hline $\mathrm{O}$ & 6.367539 & 1.240970 & 0.680234 \\
\hline $\mathrm{O}$ & 4.492885 & 9.179680 & 1.762001 \\
\hline $\mathrm{Zn}$ & 6.249136 & 8.726827 & 0.960138 \\
\hline $\mathrm{O}$ & 6.247023 & 9.043177 & -1.025245 \\
\hline $\mathrm{O}$ & 1.184038 & 9.226608 & 1.218222 \\
\hline $\mathrm{Zn}$ & 9.059399 & 10.995624 & 2.157190 \\
\hline $\mathrm{O}$ & 9.142195 & 10.813491 & 4.106835 \\
\hline $\mathrm{Zn}$ & 9.699364 & 9.001793 & 4.630914 \\
\hline $\mathrm{O}$ & 8.899783 & 7.482847 & 3.656585 \\
\hline $\mathrm{Zn}$ & 10.235648 & 12.217772 & 4.883857 \\
\hline $\mathrm{O}$ & 13.395924 & 13.239346 & 1.391076 \\
\hline $\mathrm{Zn}$ & 14.941413 & 13.560459 & 2.549372 \\
\hline $\mathrm{O}$ & 14.579087 & 13.307956 & 4.478057 \\
\hline $\mathrm{Zn}$ & 15.450006 & 11.636378 & 5.065362 \\
\hline $\mathrm{O}$ & 14.413964 & 10.187072 & 5.875965 \\
\hline $\mathrm{Zn}$ & 15.153991 & 8.373066 & 5.882153 \\
\hline $\mathrm{O}$ & 17.094743 & 8.127589 & 5.891173 \\
\hline $\mathrm{Zn}$ & 17.892826 & 9.601957 & 4.799439 \\
\hline $\mathrm{Zn}$ & 17.368056 & 11.807652 & 2.456858 \\
\hline $\mathrm{O}$ & 17.419925 & 10.287385 & 1.216088 \\
\hline $\mathrm{O}$ & 16.721067 & 13.517334 & 1.697767 \\
\hline $\mathrm{O}$ & 17.279482 & 11.385178 & 4.371944 \\
\hline $\mathrm{O}$ & 14.055123 & 6.831135 & 5.398353 \\
\hline $\mathrm{Zn}$ & 14.891752 & 5.443196 & 4.306791 \\
\hline $\mathrm{O}$ & 13.678691 & 4.264683 & 3.301107 \\
\hline $\mathrm{Zn}$ & 11.924405 & 4.292354 & 4.128520 \\
\hline $\mathrm{O}$ & 11.028056 & 5.354223 & 5.551410 \\
\hline $\mathrm{O}$ & 7.692568 & 4.821446 & 4.026785 \\
\hline $\mathrm{O}$ & -0.628248 & 1.982297 & -2.560679 \\
\hline $\mathrm{Zn}$ & -1.241530 & 3.765932 & -2.987817 \\
\hline $\mathrm{O}$ & -0.446789 & 5.242969 & -4.078938 \\
\hline $\mathrm{Zn}$ & 1.493861 & 4.996405 & -4.072199 \\
\hline $\mathrm{O}$ & 2.074407 & 0.061425 & -2.667122 \\
\hline $\mathrm{Zn}$ & 3.935339 & 0.056937 & -3.173186 \\
\hline $\mathrm{O}$ & 4.933227 & 1.307325 & -4.403553 \\
\hline $\mathrm{O}$ & 7.498133 & 2.561965 & -2.291073 \\
\hline $\mathrm{O}$ & 11.380835 & 6.375759 & -2.584901 \\
\hline $\mathrm{Zn}$ & 12.194754 & 7.900770 & -3.503420 \\
\hline $\mathrm{Zn}$ & 12.851982 & 11.067450 & -3.264811 \\
\hline $\mathrm{Zn}$ & 15.718437 & 12.450004 & -2.639612 \\
\hline $\mathrm{O}$ & 15.018562 & 13.919080 & -1.461938 \\
\hline $\mathrm{Zn}$ & 14.907851 & 6.055428 & -3.300646 \\
\hline $\mathrm{O}$ & 14.166255 & 7.775374 & -3.774180 \\
\hline $\mathrm{Zn}$ & 15.276317 & 9.296759 & -3.220943 \\
\hline
\end{tabular}


$\mathrm{O}$

O

$(\mathrm{ZnO})_{36}-(\mathrm{ZnO})_{36}$ in 1-propanol

$\begin{array}{crrr}\mathrm{O} & -0.034562 & -0.177531 & 0.050652 \\ \mathrm{Zn} & -0.816635 & 1.486794 & -0.638418 \\ \mathrm{Zn} & -1.631450 & 3.393118 & -3.123216 \\ \mathrm{O} & -2.475699 & 4.816038 & -1.990422 \\ \mathrm{Zn} & -1.692239 & 4.796803 & -0.194555 \\ \mathrm{O} & -1.007825 & 6.431037 & 0.633047 \\ \mathrm{Zn} & -0.215937 & 7.860390 & -0.455587 \\ \mathrm{O} & -0.357064 & 7.639293 & -2.414465 \\ \mathrm{Zn} & 1.470116 & 7.646133 & -3.127737 \\ \mathrm{O} & 2.737253 & 8.987930 & -2.468564 \\ \mathrm{Zn} & 4.374181 & 8.670226 & -3.428889 \\ \mathrm{O} & 5.110047 & 7.407136 & -4.768116 \\ \mathrm{Zn} & 6.783292 & 7.317037 & -3.653989 \\ \mathrm{O} & 8.644771 & 8.126064 & -3.633320 \\ \mathrm{Zn} & 9.636111 & 9.584376 & -4.544985 \\ \mathrm{O} & 8.802681 & 11.051580 & -3.478782 \\ \mathrm{Zn} & 10.344777 & 11.633335 & -2.434603 \\ \mathrm{O} & 10.195467 & 12.024981 & -0.526222 \\ \mathrm{Zn} & 11.498378 & 13.311559 & 0.162226 \\ \mathrm{O} & 11.241684 & 13.968484 & 1.979052 \\ \mathrm{Zn} & 12.917006 & 13.562090 & 3.003157 \\ \mathrm{O} & 11.950203 & 12.417242 & 4.326163 \\ \mathrm{Zn} & 12.759263 & 10.640008 & 4.234434 \\ \mathrm{O} & 11.633495 & 9.081994 & 3.908892 \\ \mathrm{Zn} & 12.478297 & 7.312649 & 3.904304 \\ \mathrm{O} & 14.324262 & 7.146678 & 3.269252 \\ \mathrm{Zn} & 15.057241 & 5.469555 & 2.565799 \\ \mathrm{Zn} & 17.859752 & 7.028033 & 2.469179 \\ \mathrm{O} & 19.003073 & 8.299633 & 1.428558 \\ \mathrm{Zn} & 18.219667 & 8.318838 & -0.367328 \\ \mathrm{O} & 17.644721 & 10.066340 & -1.046686 \\ \mathrm{Zn} & 17.233697 & 10.114693 & -2.958450 \\ \mathrm{O} & 16.313146 & 8.583418 & -3.745137 \\ \mathrm{Zn} & 16.900654 & 6.848585 & -3.035772 \\ \mathrm{O} & 16.376364 & 5.239122 & -4.007199 \\ \mathrm{Zn} & 15.358293 & 4.021426 & -2.777308 \\ \mathrm{O} & 13.742516 & 3.972091 & -3.952998 \\ \mathrm{Zn} & 12.127307 & 4.595486 & -3.026012 \\ \mathrm{Zn} & 9.407160 & 6.391079 & -2.952419 \\ & & & \\ & & & \\ & & & \end{array}$

$-3.703806$

$-3.942676$
14.762089

11.580242
11.133034

9.726540 


\begin{tabular}{|c|c|c|c|}
\hline $\mathrm{O}$ & 9.670756 & 6.344191 & -0.943442 \\
\hline $\mathrm{Zn}$ & 8.841725 & 7.668708 & 0.315747 \\
\hline $\mathrm{O}$ & 9.089041 & 7.553760 & 2.313538 \\
\hline $\mathrm{Zn}$ & 9.863559 & 9.186566 & 3.055913 \\
\hline $\mathrm{O}$ & 9.290992 & 10.979412 & 2.498142 \\
\hline $\mathrm{Zn}$ & 10.420317 & 12.466348 & 3.022490 \\
\hline $\mathrm{Zn}$ & 9.021701 & 10.894886 & 0.548617 \\
\hline $\mathrm{O}$ & 8.116330 & 9.413683 & -0.365641 \\
\hline $\mathrm{Zn}$ & 7.876677 & 9.659827 & -2.381555 \\
\hline $\mathrm{O}$ & 6.038818 & 8.798230 & -2.345424 \\
\hline $\mathrm{Zn}$ & 6.171708 & 8.540850 & -0.343815 \\
\hline $\mathrm{O}$ & 6.856616 & 6.771587 & 0.381459 \\
\hline $\mathrm{Zn}$ & 7.685641 & 5.447054 & -0.877711 \\
\hline $\mathrm{O}$ & 7.438332 & 5.561938 & -2.875506 \\
\hline $\mathrm{Zn}$ & 6.663797 & 3.929159 & -3.617916 \\
\hline $\mathrm{O}$ & 4.893842 & 4.033722 & -4.470859 \\
\hline $\mathrm{Zn}$ & 4.049037 & 5.803067 & -4.466277 \\
\hline $\mathrm{O}$ & 2.203066 & 5.969025 & -3.831241 \\
\hline $\mathrm{Zn}$ & 1.093923 & 4.445142 & -4.371738 \\
\hline $\mathrm{O}$ & -0.845340 & 4.718047 & -4.414190 \\
\hline $\mathrm{Zn}$ & -1.332419 & 6.087653 & -3.031061 \\
\hline $\mathrm{Zn}$ & 0.087873 & -0.078116 & 1.982418 \\
\hline $\mathrm{O}$ & -0.697796 & 1.245903 & 3.265875 \\
\hline $\mathrm{Zn}$ & -0.706221 & 3.000925 & 2.396482 \\
\hline $\mathrm{O}$ & 0.214328 & 4.532212 & 3.183151 \\
\hline $\mathrm{Zn}$ & -0.373201 & 6.267057 & 2.473846 \\
\hline $\mathrm{O}$ & 0.151049 & 7.876523 & 3.445287 \\
\hline $\mathrm{Zn}$ & 1.169094 & 9.094238 & 2.215387 \\
\hline $\mathrm{O}$ & 1.046702 & 9.172466 & 0.287273 \\
\hline $\mathrm{Zn}$ & 2.852776 & 9.196337 & -0.514941 \\
\hline $\mathrm{O}$ & 4.467102 & 8.980289 & 0.565726 \\
\hline $\mathrm{Zn}$ & 4.400096 & 8.520196 & 2.464021 \\
\hline $\mathrm{O}$ & 5.475851 & 7.165366 & 3.393420 \\
\hline $\mathrm{Zn}$ & 4.682493 & 5.745036 & 4.489533 \\
\hline $\mathrm{O}$ & 5.258089 & 3.914370 & 4.949102 \\
\hline $\mathrm{Zn}$ & 3.984382 & 2.555661 & 4.331333 \\
\hline $\mathrm{O}$ & 2.146564 & 2.559211 & 5.037458 \\
\hline $\mathrm{Zn}$ & 1.620734 & 4.392616 & 4.546176 \\
\hline $\mathrm{Zn}$ & 1.928749 & 7.619085 & 4.347256 \\
\hline $\mathrm{O}$ & 2.784893 & 9.143566 & 3.391041 \\
\hline $\mathrm{Zn}$ & 1.614109 & -0.497956 & -0.959421 \\
\hline $\mathrm{Zn}$ & 0.836323 & 1.214731 & -3.496041 \\
\hline $\mathrm{O}$ & 1.857198 & 2.640979 & -4.375150 \\
\hline $\mathrm{Zn}$ & 3.768050 & 2.475725 & -4.796420 \\
\hline $\mathrm{O}$ & 4.577163 & 0.698516 & -4.888278 \\
\hline
\end{tabular}




\begin{tabular}{|c|c|c|c|}
\hline $\mathrm{Zn}$ & 3.610326 & -0.446316 & -3.565301 \\
\hline $\mathrm{O}$ & 5.285639 & -0.852757 & -2.541167 \\
\hline $\mathrm{Zn}$ & 5.029166 & -0.195855 & -0.724303 \\
\hline $\mathrm{O}$ & 6.332098 & 1.090689 & -0.035835 \\
\hline $\mathrm{Zn}$ & 6.182695 & 1.482315 & 1.872551 \\
\hline $\mathrm{O}$ & 4.406108 & 1.537362 & 2.708147 \\
\hline $\mathrm{Zn}$ & 3.204978 & 0.156382 & 2.004382 \\
\hline $\mathrm{O}$ & 3.246197 & -0.236027 & 0.092016 \\
\hline $\mathrm{O}$ & 1.697632 & -0.399399 & 3.113578 \\
\hline $\mathrm{Zn}$ & 1.082282 & 1.171780 & 4.200318 \\
\hline $\mathrm{O}$ & 2.745071 & 5.949105 & 4.888377 \\
\hline $\mathrm{Zn}$ & 7.120218 & 6.724604 & 2.390439 \\
\hline $\mathrm{O}$ & 7.882614 & 4.989617 & 3.071325 \\
\hline $\mathrm{Zn}$ & 8.650720 & 3.455889 & 1.819529 \\
\hline $\mathrm{O}$ & 7.724774 & 2.064093 & 2.916756 \\
\hline $\mathrm{Zn}$ & 6.891336 & 3.531262 & 3.982998 \\
\hline $\mathrm{Zn}$ & 9.744086 & 5.798659 & 3.092017 \\
\hline $\mathrm{O}$ & 10.488579 & 4.317505 & 1.783433 \\
\hline $\mathrm{Zn}$ & 10.355682 & 4.574935 & -0.218174 \\
\hline $\mathrm{O}$ & 12.060284 & 4.135437 & -1.127700 \\
\hline $\mathrm{Zn}$ & 13.674577 & 3.919349 & -0.046999 \\
\hline $\mathrm{O}$ & 15.480665 & 3.943199 & -0.849196 \\
\hline $\mathrm{Zn}$ & 16.743312 & 5.255269 & -0.106343 \\
\hline $\mathrm{O}$ & 16.884422 & 5.476388 & 1.852535 \\
\hline $\mathrm{O}$ & 11.417324 & 5.708552 & 4.206159 \\
\hline $\mathrm{Zn}$ & 12.153194 & 4.445449 & 2.866947 \\
\hline $\mathrm{O}$ & 13.790122 & 4.127734 & 1.906623 \\
\hline $\mathrm{O}$ & 8.411083 & 3.702077 & -0.196374 \\
\hline $\mathrm{Zn}$ & 7.505779 & 2.220869 & -1.110680 \\
\hline $\mathrm{Zn}$ & 6.107028 & 0.649383 & -3.584580 \\
\hline $\mathrm{O}$ & 7.236362 & 2.136296 & -3.060185 \\
\hline $\mathrm{O}$ & 1.783256 & -0.414578 & -2.917365 \\
\hline $\mathrm{O}$ & -1.117263 & 3.049287 & 0.484722 \\
\hline $\mathrm{O}$ & -0.838529 & 1.706997 & -2.588730 \\
\hline $\mathrm{O}$ & 13.281377 & 13.351670 & -0.654003 \\
\hline $\mathrm{Zn}$ & 13.322530 & 12.959262 & -2.566372 \\
\hline $\mathrm{O}$ & 12.121386 & 11.578300 & -3.270149 \\
\hline $\mathrm{Zn}$ & 12.543093 & 10.559984 & -4.893328 \\
\hline $\mathrm{O}$ & 11.269354 & 9.201304 & -5.511093 \\
\hline $\mathrm{Zn}$ & 11.844917 & 7.370639 & -5.051483 \\
\hline $\mathrm{O}$ & 11.051532 & 5.950314 & -3.955389 \\
\hline $\mathrm{Zn}$ & 14.913460 & 13.613545 & 0.397479 \\
\hline $\mathrm{O}$ & 14.744153 & 13.530343 & 2.355426 \\
\hline $\mathrm{Zn}$ & 15.691003 & 11.900973 & 2.934098 \\
\hline $\mathrm{O}$ & 14.670128 & 10.474719 & 3.813192 \\
\hline
\end{tabular}




$\begin{array}{crrr}\mathrm{Zn} & 15.433399 & 8.670559 & 3.809781 \\ \mathrm{O} & 17.372662 & 8.397638 & 3.852294 \\ \mathrm{Zn} & 18.158793 & 9.722558 & 2.561322 \\ \mathrm{Zn} & 17.344098 & 11.628818 & 0.076473 \\ \mathrm{Zn} & 16.439635 & 13.193747 & -2.544409 \\ \mathrm{O} & 17.225278 & 11.869708 & -3.827856 \\ \mathrm{Zn} & 15.445201 & 11.943856 & -4.762301 \\ \mathrm{Zn} & 14.906735 & 8.723021 & -5.108158 \\ \mathrm{O} & 13.782344 & 7.166557 & -5.450304 \\ \mathrm{Zn} & 14.598670 & 5.496579 & -4.909184 \\ \mathrm{O} & 16.562095 & 13.293155 & -0.612642 \\ \mathrm{O} & 17.365850 & 11.408657 & 2.026784 \\ \mathrm{O} & 17.535227 & 6.684618 & -1.194961 \\ \mathrm{O} & 14.829874 & 13.515044 & -3.675568 \\ \mathrm{O} & 14.380912 & 10.556428 & -5.599443\end{array}$

$(\mathrm{ZnO})_{36}-(\mathrm{ZnO})_{36}$ in 1-butanol

$\begin{array}{crrr}\mathrm{O} & 0.024995 & -0.084187 & 0.041427 \\ \mathrm{Zn} & -1.747089 & -0.125558 & -0.735938 \\ \mathrm{Zn} & -1.191085 & -2.883488 & -2.287325 \\ \mathrm{O} & -2.310051 & -1.284463 & -2.268128 \\ \mathrm{Zn} & -3.948103 & -1.631752 & -1.187611 \\ \mathrm{O} & -4.731836 & -3.337633 & -0.727018 \\ \mathrm{Zn} & -5.213113 & -3.810692 & 1.117712 \\ \mathrm{O} & -5.773191 & -5.599590 & 1.677232 \\ \mathrm{Zn} & -5.975100 & -5.652334 & 3.603198 \\ \mathrm{O} & -5.405370 & -7.184752 & 4.758362 \\ \mathrm{Zn} & -3.994038 & -8.285096 & 3.966442 \\ \mathrm{Zn} & -2.694675 & -9.707398 & 1.295207 \\ \mathrm{Zn} & -2.413778 & -11.579172 & -0.871849 \\ \mathrm{O} & -3.039774 & -12.980469 & -2.146928 \\ \mathrm{Zn} & -1.222438 & -12.961740 & -2.945734 \\ \mathrm{O} & -0.548636 & -11.443182 & -1.834593 \\ \mathrm{Zn} & 1.248300 & -11.319505 & -0.924131 \\ \mathrm{O} & 0.998480 & -9.195685 & -0.769162 \\ \mathrm{Zn} & -7.632363 & -1.297358 \\ \mathrm{O} & 2.070599 & -7.310797 & -0.380078 \\ \mathrm{Zn} & 3.779516 & -5.553999 & -0.781788 \\ \mathrm{Zn} & 4.488326 & -2.839097 & -0.630031 \\ \mathrm{O} & 4.208199 & -1.401086 & 0.107215 \\ \mathrm{Zn} & -1.349787 & -1.688827 \\ \mathrm{O} & 1.129524 & -2.796209 & -2.169597 \\ \mathrm{Zn} & -4.348259 & \end{array}$




\begin{tabular}{|c|c|c|c|}
\hline $\mathrm{O}$ & 1.097356 & -6.156997 & -2.141253 \\
\hline $\mathrm{Zn}$ & -0.814433 & -6.219089 & -2.557758 \\
\hline $\mathrm{Zn}$ & -3.158653 & -8.003383 & -1.806843 \\
\hline $\mathrm{O}$ & -2.609033 & -9.558116 & -0.708262 \\
\hline $\mathrm{Zn}$ & -0.768223 & -9.424159 & -1.749004 \\
\hline $\mathrm{O}$ & -1.526282 & -7.998079 & -2.941837 \\
\hline $\mathrm{Zn}$ & -0.088373 & -0.089054 & 2.001423 \\
\hline $\mathrm{O}$ & -1.749837 & -0.674668 & 2.857166 \\
\hline $\mathrm{Zn}$ & -3.403088 & -1.024693 & 1.866411 \\
\hline $\mathrm{O}$ & -3.554216 & -0.149427 & 0.121693 \\
\hline $\mathrm{O}$ & 1.669960 & -0.073765 & 2.889515 \\
\hline $\mathrm{Zn}$ & 1.523104 & -0.193909 & 4.819359 \\
\hline $\mathrm{Zn}$ & 0.569132 & -1.826097 & 6.762189 \\
\hline $\mathrm{Zn}$ & -1.683111 & -1.107296 & 4.767021 \\
\hline $\mathrm{O}$ & -2.738486 & -2.638762 & 5.369898 \\
\hline $\mathrm{Zn}$ & -4.360775 & -2.993860 & 4.340790 \\
\hline $\mathrm{Zn}$ & -4.626844 & -5.763992 & 5.931378 \\
\hline $\mathrm{Zn}$ & -1.726288 & -7.285540 & 6.283655 \\
\hline $\mathrm{O}$ & -2.411584 & -8.647567 & 5.058636 \\
\hline $\mathrm{Zn}$ & -1.034992 & -9.757076 & 4.192462 \\
\hline $\mathrm{O}$ & 0.761597 & -9.632740 & 5.103342 \\
\hline $\mathrm{Zn}$ & 0.981733 & -11.651493 & 5.018542 \\
\hline $\mathrm{O}$ & -0.784265 & -11.880191 & 4.038044 \\
\hline $\mathrm{Zn}$ & -1.857709 & -13.441581 & 4.568873 \\
\hline $\mathrm{Zn}$ & -4.275379 & -15.518379 & 4.052686 \\
\hline $\mathrm{Zn}$ & -1.630326 & -16.723487 & 5.443854 \\
\hline $\mathrm{O}$ & -0.497180 & -18.274885 & 5.124816 \\
\hline $\mathrm{Zn}$ & -1.122840 & -19.716209 & 3.960781 \\
\hline $\mathrm{Zn}$ & -2.762304 & -19.793376 & 1.212899 \\
\hline $\mathrm{O}$ & -1.465176 & -21.018125 & 0.384471 \\
\hline $\mathrm{Zn}$ & -1.316717 & -20.890704 & -1.545671 \\
\hline $\mathrm{Zn}$ & -2.749740 & -18.060137 & -1.732076 \\
\hline $\mathrm{O}$ & -2.224131 & -16.259616 & -2.295186 \\
\hline $\mathrm{Zn}$ & -0.678391 & -16.027839 & -3.474674 \\
\hline $\mathrm{O}$ & 0.095435 & -14.230534 & -3.543884 \\
\hline $\mathrm{Zn}$ & 1.937477 & -13.794702 & -3.012051 \\
\hline $\mathrm{O}$ & 3.061803 & -15.359597 & -3.426373 \\
\hline $\mathrm{Zn}$ & 2.232557 & -17.137394 & -3.385556 \\
\hline $\mathrm{O}$ & 2.946868 & -18.446176 & -2.095450 \\
\hline $\mathrm{Zn}$ & 1.892254 & -19.978243 & -1.492126 \\
\hline $\mathrm{O}$ & 1.958794 & -20.414182 & 0.416768 \\
\hline $\mathrm{Zn}$ & 0.295741 & -21.006198 & 1.267776 \\
\hline $\mathrm{O}$ & 0.190558 & -20.977050 & 3.228210 \\
\hline $\mathrm{Zn}$ & 1.961274 & -20.944007 & 4.008576 \\
\hline $\mathrm{O}$ & 2.525537 & -19.787413 & 5.542061 \\
\hline
\end{tabular}




\begin{tabular}{|c|c|c|c|}
\hline $\mathrm{Zn}$ & 4.164095 & -19.443181 & 4.461311 \\
\hline $\mathrm{O}$ & 4.944987 & -17.736477 & 3.999705 \\
\hline $\mathrm{Zn}$ & 5.423483 & -17.265928 & 2.153584 \\
\hline $\mathrm{Zn}$ & 6.187370 & -15.428488 & -0.332283 \\
\hline $\mathrm{O}$ & 5.984067 & -15.477386 & 1.593583 \\
\hline $\mathrm{Zn}$ & 5.013478 & -13.961950 & 2.419513 \\
\hline $\mathrm{O}$ & 4.296516 & -12.568306 & 1.251198 \\
\hline $\mathrm{Zn}$ & 4.206814 & -12.794782 & -0.697438 \\
\hline $\mathrm{O}$ & 2.623976 & -12.431289 & -1.789077 \\
\hline $\mathrm{Zn}$ & 2.980317 & -1.280238 & 2.058295 \\
\hline $\mathrm{O}$ & 3.555060 & -2.803219 & 3.152385 \\
\hline $\mathrm{Zn}$ & 2.961353 & -3.018607 & 5.002851 \\
\hline $\mathrm{O}$ & 2.417210 & -1.481203 & 6.068665 \\
\hline $\mathrm{Zn}$ & 4.369976 & -4.310810 & 2.219070 \\
\hline $\mathrm{O}$ & 5.226134 & -4.168238 & 0.469305 \\
\hline $\mathrm{O}$ & 3.847681 & -6.123294 & 2.755347 \\
\hline $\mathrm{Zn}$ & 3.282305 & -6.305893 & 4.614099 \\
\hline $\mathrm{O}$ & 2.435377 & -4.818946 & 5.565968 \\
\hline $\mathrm{Zn}$ & 0.889939 & -5.051654 & 6.745684 \\
\hline $\mathrm{Zn}$ & -2.023558 & -3.946063 & 6.661250 \\
\hline $\mathrm{O}$ & -2.853387 & -5.723602 & 6.701880 \\
\hline $\mathrm{Zn}$ & 3.600284 & -7.639269 & 1.560684 \\
\hline $\mathrm{Zn}$ & 0.880498 & -9.447843 & 1.230977 \\
\hline $\mathrm{Zn}$ & 2.909209 & -11.368452 & 1.973576 \\
\hline $\mathrm{Zn}$ & 2.626883 & -9.496774 & 4.140913 \\
\hline $\mathrm{Zn}$ & 1.435176 & -8.115702 & 6.216071 \\
\hline $\mathrm{O}$ & 0.115721 & -6.848706 & 6.814879 \\
\hline $\mathrm{O}$ & 2.667783 & -9.264083 & 2.138350 \\
\hline $\mathrm{O}$ & -0.871025 & -9.443398 & 2.182546 \\
\hline $\mathrm{Zn}$ & -0.665743 & -11.628343 & 2.037892 \\
\hline $\mathrm{Zn}$ & -3.386335 & -13.435116 & 1.709770 \\
\hline $\mathrm{O}$ & -3.566243 & -13.761842 & 3.650832 \\
\hline $\mathrm{O}$ & 1.085597 & -11.632297 & 1.085979 \\
\hline $\mathrm{O}$ & -2.453032 & -11.811463 & 1.130733 \\
\hline $\mathrm{O}$ & 2.822070 & -11.517838 & 3.977097 \\
\hline $\mathrm{Zn}$ & 3.372704 & -13.070746 & 5.078074 \\
\hline $\mathrm{O}$ & 4.515028 & -14.404238 & 4.269618 \\
\hline $\mathrm{Zn}$ & 3.992397 & -16.219261 & 4.782925 \\
\hline $\mathrm{O}$ & 2.195464 & -16.390569 & 5.547145 \\
\hline $\mathrm{Zn}$ & 1.028278 & -14.853062 & 5.831552 \\
\hline $\mathrm{O}$ & 1.741038 & -13.074070 & 6.214408 \\
\hline $\mathrm{O}$ & 3.252567 & -8.096891 & 5.417558 \\
\hline $\mathrm{O}$ & -0.209587 & -3.518211 & 7.290584 \\
\hline $\mathrm{O}$ & -0.239422 & -0.278027 & 5.787001 \\
\hline $\mathrm{O}$ & -4.498553 & -2.542693 & 2.440987 \\
\hline
\end{tabular}




$\begin{array}{crrr}\mathrm{O} & -5.653907 & -4.288286 & 5.041285 \\ \mathrm{Zn} & 1.406384 & -18.188626 & 5.562246 \\ \mathrm{O} & 3.769268 & -20.926012 & 3.152891 \\ \mathrm{Zn} & 3.611452 & -20.054198 & 1.406938 \\ \mathrm{O} & 4.706878 & -18.535764 & 0.832940 \\ \mathrm{Zn} & 4.569802 & -18.088273 & -1.067980 \\ \mathrm{Zn} & 4.835752 & -15.317841 & -2.658093 \\ \mathrm{O} & 5.617754 & -13.896558 & -1.488495 \\ \mathrm{O} & 5.862908 & -16.793880 & -1.768587 \\ \mathrm{O} & 0.418826 & -17.564171 & -4.016086 \\ \mathrm{Zn} & -0.359316 & -19.256362 & -3.486241 \\ \mathrm{O} & -2.208022 & -19.600641 & -2.794318 \\ \mathrm{O} & 0.447055 & -20.806039 & -2.511565 \\ \mathrm{O} & -3.338697 & -18.270998 & 0.120035 \\ \mathrm{Zn} & -4.155156 & -16.764070 & 1.053041 \\ \mathrm{Zn} & -3.995101 & -18.232776 & 3.902153 \\ \mathrm{O} & -2.915593 & -19.669383 & 3.163962 \\ \mathrm{O} & -3.633224 & -14.951711 & 0.516025 \\ \mathrm{Zn} & -3.069479 & -14.771619 & -1.343512 \\ \mathrm{O} & -5.013213 & -16.904500 & 2.802107 \\ \mathrm{O} & -1.981033 & -4.681187 & -2.272277 \\ \mathrm{Zn} & -3.778851 & -4.854811 & -1.510632 \\ \mathrm{O} & -4.301171 & -6.670067 & -0.998108 \\ \mathrm{Zn} & -4.801302 & -7.114133 & 0.851073 \\ \mathrm{O} & -4.082565 & -8.508326 & 2.017670 \\ \mathrm{O} & 3.786629 & -4.182135 & -2.055386 \\ \mathrm{O} & -0.883480 & -14.914966 & 5.414959 \\ \mathrm{O} & -3.573097 & -16.889371 & 5.326985\end{array}$

$(\mathrm{ZnO})_{36}-(\mathrm{ZnO})_{36}$ in 1-pentanol

$\begin{array}{crrr}\mathrm{O} & 0.008289 & -0.209273 & -0.014475 \\ \mathrm{Zn} & -1.739511 & -0.292564 & -0.852521 \\ \mathrm{Zn} & -0.918966 & -2.858144 & -2.420347 \\ \mathrm{O} & -2.244395 & -1.433261 & -2.417203 \\ \mathrm{Zn} & -3.823133 & -1.968518 & -1.309191 \\ \mathrm{O} & -4.455998 & -3.757657 & -0.952289 \\ \mathrm{Zn} & -4.950256 & -4.276112 & 0.873437 \\ \mathrm{O} & -5.479396 & -6.085205 & 1.402485 \\ \mathrm{Zn} & -5.704778 & -6.226124 & 3.325027 \\ \mathrm{Zn} & -3.531264 & -8.676564 & 3.628134 \\ \mathrm{O} & -5.033975 & -7.744260 & 4.453397 \\ \mathrm{Zn} & -4.370106 & -6.329257 & 5.686293 \\ \mathrm{Zn} & -1.403983 & -7.582626 & 6.011595\end{array}$




\begin{tabular}{|c|c|c|c|}
\hline $\mathrm{O}$ & -1.929811 & -8.939482 & 4.709543 \\
\hline $\mathrm{Zn}$ & -0.472755 & -9.943741 & 3.841167 \\
\hline $\mathrm{O}$ & 1.272014 & -9.811626 & 4.810753 \\
\hline $\mathrm{Zn}$ & 1.568080 & -11.827231 & 4.615819 \\
\hline $\mathrm{O}$ & -0.162294 & -12.059991 & 3.591849 \\
\hline $\mathrm{Zn}$ & -1.090435 & -13.728236 & 4.099846 \\
\hline $\mathrm{Zn}$ & -3.318859 & -15.920139 & 3.417838 \\
\hline $\mathrm{Zn}$ & -0.659274 & -17.069529 & 4.935200 \\
\hline $\mathrm{O}$ & 0.602350 & -18.543842 & 4.665493 \\
\hline $\mathrm{Zn}$ & 0.111177 & -19.903659 & 3.345327 \\
\hline $\mathrm{Zn}$ & -1.408663 & -19.898852 & 0.495033 \\
\hline $\mathrm{O}$ & -0.004829 & -20.978563 & -0.346954 \\
\hline $\mathrm{Zn}$ & 0.268900 & -20.670925 & -2.242978 \\
\hline $\mathrm{Zn}$ & -1.556849 & -18.014871 & -2.314533 \\
\hline $\mathrm{O}$ & -1.058937 & -16.190526 & -2.829165 \\
\hline $\mathrm{Zn}$ & 0.472724 & -15.855563 & -3.987547 \\
\hline $\mathrm{Zn}$ & -0.195484 & -12.845055 & -3.397509 \\
\hline $\mathrm{O}$ & -2.045334 & -12.966942 & -2.705544 \\
\hline $\mathrm{Zn}$ & -1.538717 & -11.608616 & -1.332107 \\
\hline $\mathrm{O}$ & -1.716090 & -11.967324 & 0.663106 \\
\hline $\mathrm{Zn}$ & -2.547378 & -13.661766 & 1.232138 \\
\hline $\mathrm{O}$ & -2.763545 & -14.088540 & 3.137319 \\
\hline $\mathrm{Zn}$ & -0.097370 & -0.274127 & 1.934739 \\
\hline $\mathrm{O}$ & -1.621809 & -1.101747 & 2.819370 \\
\hline $\mathrm{Zn}$ & -3.207681 & -1.502431 & 1.745073 \\
\hline $\mathrm{O}$ & -3.496196 & -0.543369 & 0.077611 \\
\hline $\mathrm{Zn}$ & 1.498760 & -1.211437 & -0.806369 \\
\hline $\mathrm{O}$ & 3.247247 & -1.213765 & 0.090514 \\
\hline $\mathrm{Zn}$ & 4.524672 & -2.516040 & -0.557434 \\
\hline $\mathrm{O}$ & 5.616643 & -3.857107 & 0.447168 \\
\hline $\mathrm{Zn}$ & 4.928818 & -5.194923 & -0.878910 \\
\hline $\mathrm{O}$ & 4.373572 & -7.026545 & -0.598398 \\
\hline $\mathrm{Zn}$ & 2.700468 & -7.386885 & -1.560929 \\
\hline $\mathrm{O}$ & 1.702725 & -5.914012 & -2.380594 \\
\hline $\mathrm{Zn}$ & 2.269213 & -4.045561 & -2.396262 \\
\hline $\mathrm{O}$ & 4.182581 & -3.772099 & -2.072446 \\
\hline $\mathrm{O}$ & 1.614748 & -0.136540 & 2.885945 \\
\hline $\mathrm{Zn}$ & 1.341023 & -0.444187 & 4.781971 \\
\hline $\mathrm{Zn}$ & -1.676160 & -1.518938 & 4.731620 \\
\hline $\mathrm{Zn}$ & -4.193262 & -3.535070 & 4.152128 \\
\hline $\mathrm{O}$ & -5.446616 & -4.886870 & 4.794121 \\
\hline $\mathrm{Zn}$ & 3.018589 & -1.216221 & 2.043928 \\
\hline $\mathrm{O}$ & 3.831040 & -2.682530 & 3.062779 \\
\hline $\mathrm{Zn}$ & 3.166798 & -3.100190 & 4.853519 \\
\hline $\mathrm{O}$ & 2.370825 & -1.690108 & 5.954316 \\
\hline
\end{tabular}




\begin{tabular}{|c|c|c|c|}
\hline $\mathrm{Zn}$ & 0.586596 & -2.143951 & 6.751917 \\
\hline $\mathrm{O}$ & -0.092648 & -3.892609 & 7.196586 \\
\hline $\mathrm{Zn}$ & -1.898329 & -4.403984 & 6.598744 \\
\hline $\mathrm{O}$ & -2.679852 & -6.204099 & 6.612296 \\
\hline $\mathrm{Zn}$ & 4.665712 & -4.177809 & 2.111142 \\
\hline $\mathrm{O}$ & 4.162500 & -5.997686 & 2.613357 \\
\hline $\mathrm{Zn}$ & 3.663159 & -6.357220 & 4.466668 \\
\hline $\mathrm{O}$ & 2.668880 & -4.924527 & 5.368170 \\
\hline $\mathrm{Zn}$ & 1.137271 & -5.259644 & 6.526588 \\
\hline $\mathrm{O}$ & 0.416147 & -7.082517 & 6.541791 \\
\hline $\mathrm{Zn}$ & 1.805570 & -8.270257 & 5.936719 \\
\hline $\mathrm{O}$ & 3.655244 & -8.148012 & 5.244321 \\
\hline $\mathrm{Zn}$ & 3.148608 & -9.506468 & 3.871038 \\
\hline $\mathrm{O}$ & 3.368432 & -11.502129 & 3.587532 \\
\hline $\mathrm{Zn}$ & 4.105214 & -13.003730 & 4.652408 \\
\hline $\mathrm{O}$ & 5.446441 & -14.106218 & 3.797524 \\
\hline $\mathrm{Zn}$ & 5.973972 & -13.620272 & 1.964589 \\
\hline $\mathrm{Zn}$ & 6.560246 & -16.839054 & 1.665542 \\
\hline $\mathrm{Zn}$ & 7.314819 & -14.889049 & -0.786043 \\
\hline $\mathrm{O}$ & 7.089395 & -15.029964 & 1.136495 \\
\hline $\mathrm{Zn}$ & 4.157344 & -7.453329 & 1.306777 \\
\hline $\mathrm{Zn}$ & 1.562260 & -9.428566 & 0.931497 \\
\hline $\mathrm{O}$ & 1.772370 & -9.055156 & -1.052931 \\
\hline $\mathrm{Zn}$ & 2.082822 & -11.171422 & -1.302217 \\
\hline $\mathrm{Zn}$ & 0.041924 & -9.287965 & -2.076777 \\
\hline $\mathrm{Zn}$ & -2.495199 & -8.111380 & -2.113481 \\
\hline $\mathrm{O}$ & -1.758467 & -9.612964 & -1.048555 \\
\hline $\mathrm{Zn}$ & -2.006574 & -9.861654 & 0.927112 \\
\hline $\mathrm{O}$ & -3.537592 & -8.882168 & 1.680385 \\
\hline $\mathrm{Zn}$ & -4.363951 & -7.494876 & 0.574381 \\
\hline $\mathrm{O}$ & -3.836427 & -7.008922 & -1.258556 \\
\hline $\mathrm{Zn}$ & -3.331551 & -5.176511 & -1.722559 \\
\hline $\mathrm{O}$ & -1.511511 & -4.721017 & -2.269814 \\
\hline $\mathrm{Zn}$ & -0.215179 & -6.102303 & -2.761278 \\
\hline $\mathrm{O}$ & -0.855938 & -7.885695 & -3.207241 \\
\hline $\mathrm{O}$ & 3.326105 & -9.147784 & 1.875831 \\
\hline $\mathrm{Zn}$ & 3.616616 & -11.253464 & 1.611867 \\
\hline $\mathrm{O}$ & 5.147633 & -12.232960 & 0.858601 \\
\hline $\mathrm{Zn}$ & 5.141330 & -12.438575 & -1.089144 \\
\hline $\mathrm{O}$ & 3.539877 & -12.175690 & -2.170563 \\
\hline $\mathrm{Zn}$ & 3.014070 & -13.532590 & -3.472573 \\
\hline $\mathrm{O}$ & 1.193932 & -14.032720 & -4.002719 \\
\hline $\mathrm{O}$ & -0.208373 & -9.539842 & 1.853077 \\
\hline $\mathrm{Zn}$ & 0.047787 & -11.686582 & 1.607419 \\
\hline $\mathrm{O}$ & 1.818439 & -11.575308 & 0.685876 \\
\hline
\end{tabular}




\begin{tabular}{|c|c|c|c|}
\hline $\mathrm{O}$ & -0.341057 & -0.620958 & 5.843331 \\
\hline $\mathrm{O}$ & -2.621796 & -3.151070 & 5.253843 \\
\hline $\mathrm{O}$ & -4.264122 & -3.063215 & 2.261216 \\
\hline $\mathrm{Zn}$ & 1.825197 & -15.012860 & 5.300260 \\
\hline $\mathrm{O}$ & 2.466005 & -13.229485 & 5.746236 \\
\hline $\mathrm{Zn}$ & 4.941553 & -15.938627 & 4.261530 \\
\hline $\mathrm{O}$ & 6.065981 & -17.357503 & 3.491268 \\
\hline $\mathrm{Zn}$ & 5.433092 & -19.146637 & 3.848164 \\
\hline $\mathrm{O}$ & 3.854344 & -19.681888 & 4.956163 \\
\hline $\mathrm{Zn}$ & 3.349457 & -20.822573 & 3.391470 \\
\hline $\mathrm{O}$ & 1.601643 & -20.905850 & 2.553452 \\
\hline $\mathrm{Zn}$ & 1.707294 & -20.840986 & 0.604237 \\
\hline $\mathrm{O}$ & 3.231737 & -20.013362 & -0.280380 \\
\hline $\mathrm{Zn}$ & 3.286117 & -19.596194 & -2.192642 \\
\hline $\mathrm{O}$ & 4.231820 & -17.964109 & -2.714873 \\
\hline $\mathrm{Zn}$ & 3.508355 & -16.711191 & -4.059775 \\
\hline $\mathrm{O}$ & 1.702662 & -17.222561 & -4.657579 \\
\hline $\mathrm{Zn}$ & 1.023386 & -18.971212 & -4.212939 \\
\hline $\mathrm{O}$ & -0.760861 & -19.424955 & -3.415320 \\
\hline $\mathrm{O}$ & 3.121514 & -16.394145 & 4.808769 \\
\hline $\mathrm{Zn}$ & 2.528919 & -18.257002 & 4.959309 \\
\hline $\mathrm{O}$ & 5.106149 & -20.571775 & 2.461349 \\
\hline $\mathrm{Zn}$ & 4.817635 & -19.612709 & 0.793888 \\
\hline $\mathrm{O}$ & 5.874103 & -18.051942 & 0.277755 \\
\hline $\mathrm{Zn}$ & 5.803276 & -17.580089 & -1.613159 \\
\hline $\mathrm{Zn}$ & 5.980152 & -14.785902 & -3.147309 \\
\hline $\mathrm{O}$ & 4.289920 & -14.911095 & -4.073342 \\
\hline $\mathrm{O}$ & 7.056650 & -16.228303 & -2.255141 \\
\hline $\mathrm{O}$ & 6.644023 & -13.370906 & -1.914410 \\
\hline $\mathrm{O}$ & 1.950999 & -20.494200 & -3.304317 \\
\hline $\mathrm{O}$ & -2.221120 & -18.432542 & -0.523808 \\
\hline $\mathrm{Zn}$ & -3.055776 & -16.937209 & 0.427742 \\
\hline $\mathrm{Zn}$ & -2.914720 & -18.599008 & 3.096347 \\
\hline $\mathrm{O}$ & -1.637307 & -19.901313 & 2.448448 \\
\hline $\mathrm{O}$ & -2.552586 & -15.117335 & -0.074512 \\
\hline $\mathrm{Zn}$ & -2.053270 & -14.757729 & -1.927832 \\
\hline $\mathrm{O}$ & -4.006671 & -17.257925 & 2.091742 \\
\hline $\mathrm{O}$ & 0.337972 & -11.303597 & -2.271644 \\
\hline $\mathrm{O}$ & 1.007596 & -2.571250 & -2.126545 \\
\hline $\mathrm{O}$ & -0.092698 & -15.201099 & 4.919535 \\
\hline $\mathrm{O}$ & -2.572644 & -17.342972 & 4.611374 \\
\hline
\end{tabular}

$(\mathrm{ZnO})_{36}-(\mathrm{ZnO})_{36}$ in 1-octanol 


\begin{tabular}{|c|c|c|c|}
\hline $\mathrm{O}$ & -0.008489 & -0.216138 & 0.021660 \\
\hline $\mathrm{Zn}$ & -1.737572 & -0.261015 & -0.842648 \\
\hline $\mathrm{Zn}$ & -0.904261 & -2.839080 & -2.420821 \\
\hline $\mathrm{O}$ & -2.225387 & -1.412808 & -2.390913 \\
\hline $\mathrm{Zn}$ & -3.865464 & -1.852979 & -1.334295 \\
\hline $\mathrm{O}$ & -4.419332 & -3.645481 & -0.881106 \\
\hline $\mathrm{Zn}$ & -4.921054 & -4.139834 & 0.943578 \\
\hline $\mathrm{O}$ & -5.413728 & -5.955592 & 1.460337 \\
\hline $\mathrm{Zn}$ & -5.750733 & -6.178718 & 3.360096 \\
\hline $\mathrm{O}$ & -5.008218 & -7.676493 & 4.462607 \\
\hline $\mathrm{Zn}$ & -3.503203 & -8.603856 & 3.640560 \\
\hline $\mathrm{O}$ & -3.508362 & -8.791000 & 1.691278 \\
\hline $\mathrm{Zn}$ & -2.030996 & -9.844939 & 0.915616 \\
\hline $\mathrm{Zn}$ & -1.605169 & -11.636132 & -1.321334 \\
\hline $\mathrm{O}$ & -2.093846 & -13.002790 & -2.686415 \\
\hline $\mathrm{Zn}$ & -0.264871 & -12.819379 & -3.422852 \\
\hline $\mathrm{O}$ & 0.267630 & -11.303408 & -2.267359 \\
\hline $\mathrm{Zn}$ & 2.032140 & -11.173154 & -1.328711 \\
\hline $\mathrm{O}$ & 1.713769 & -9.049003 & -1.090675 \\
\hline $\mathrm{Zn}$ & 2.625012 & -7.377277 & -1.600786 \\
\hline $\mathrm{O}$ & 4.274366 & -6.955662 & -0.621007 \\
\hline $\mathrm{Zn}$ & 4.908490 & -5.159902 & -0.955672 \\
\hline $\mathrm{Zn}$ & 4.508192 & -2.488737 & -0.604714 \\
\hline $\mathrm{O}$ & 3.244512 & -1.193669 & 0.083823 \\
\hline $\mathrm{Zn}$ & 1.485708 & -1.188046 & -0.784973 \\
\hline $\mathrm{O}$ & 1.011543 & -2.554973 & -2.103090 \\
\hline $\mathrm{Zn}$ & 2.248522 & -4.040244 & -2.413015 \\
\hline $\mathrm{O}$ & 1.635678 & -5.892342 & -2.406238 \\
\hline $\mathrm{Zn}$ & -0.279759 & -6.100900 & -2.773730 \\
\hline $\mathrm{Zn}$ & -2.567810 & -8.088535 & -2.069656 \\
\hline $\mathrm{O}$ & -1.875009 & -9.644876 & -1.072196 \\
\hline $\mathrm{Zn}$ & -0.052515 & -9.301495 & -2.065042 \\
\hline $\mathrm{O}$ & -0.934121 & -7.882610 & -3.173012 \\
\hline $\mathrm{Zn}$ & -0.183694 & -0.240763 & 1.969754 \\
\hline $\mathrm{O}$ & -1.823602 & -0.818206 & 2.858027 \\
\hline $\mathrm{Zn}$ & -3.411051 & -1.242027 & 1.787206 \\
\hline $\mathrm{O}$ & -3.528435 & -0.406742 & 0.024036 \\
\hline $\mathrm{O}$ & 1.552973 & -0.226196 & 2.880807 \\
\hline $\mathrm{Zn}$ & 1.329872 & -0.394242 & 4.794246 \\
\hline $\mathrm{Zn}$ & -1.755455 & -1.387945 & 4.725265 \\
\hline $\mathrm{Zn}$ & -4.263740 & -3.423247 & 4.164923 \\
\hline $\mathrm{Zn}$ & -4.363188 & -6.242764 & 5.684858 \\
\hline $\mathrm{O}$ & -2.616001 & -6.143144 & 6.500799 \\
\hline $\mathrm{Zn}$ & -1.351005 & -7.573582 & 6.008179 \\
\hline $\mathrm{O}$ & -1.896323 & -8.914133 & 4.700308 \\
\hline
\end{tabular}




\begin{tabular}{|c|c|c|c|}
\hline $\mathrm{Zn}$ & -0.448084 & -9.917581 & 3.821765 \\
\hline $\mathrm{O}$ & 1.319623 & -9.786141 & 4.755253 \\
\hline $\mathrm{Zn}$ & 1.639917 & -11.785930 & 4.554705 \\
\hline $\mathrm{O}$ & -0.128745 & -12.038719 & 3.583077 \\
\hline $\mathrm{Zn}$ & -1.061183 & -13.701573 & 4.083088 \\
\hline $\mathrm{Zn}$ & -3.362728 & -15.899706 & 3.411540 \\
\hline $\mathrm{Zn}$ & -0.713337 & -17.026384 & 4.897074 \\
\hline $\mathrm{O}$ & 0.506273 & -18.525353 & 4.589517 \\
\hline $\mathrm{Zn}$ & 0.031801 & -19.893588 & 3.274613 \\
\hline $\mathrm{Zn}$ & -1.491028 & -19.858764 & 0.455516 \\
\hline $\mathrm{O}$ & -0.050019 & -20.866796 & -0.401927 \\
\hline $\mathrm{Zn}$ & 0.139139 & -20.700957 & -2.321026 \\
\hline $\mathrm{Zn}$ & -1.603467 & -18.010383 & -2.379325 \\
\hline $\mathrm{O}$ & -1.107147 & -16.187869 & -2.890321 \\
\hline $\mathrm{Zn}$ & 0.432267 & -15.857485 & -4.038702 \\
\hline $\mathrm{O}$ & 1.104771 & -14.018052 & -4.048162 \\
\hline $\mathrm{Zn}$ & 2.919517 & -13.516618 & -3.515382 \\
\hline $\mathrm{O}$ & 4.178052 & -14.962376 & -3.988297 \\
\hline $\mathrm{Zn}$ & 3.440859 & -16.773036 & -3.991588 \\
\hline $\mathrm{O}$ & 4.188609 & -18.074051 & -2.711651 \\
\hline $\mathrm{Zn}$ & 3.276868 & -19.730804 & -2.194269 \\
\hline $\mathrm{O}$ & 3.340659 & -20.266516 & -0.321762 \\
\hline $\mathrm{Zn}$ & 1.682493 & -20.864001 & 0.515501 \\
\hline $\mathrm{O}$ & 1.513529 & -20.882893 & 2.466223 \\
\hline $\mathrm{Zn}$ & 3.242120 & -20.833080 & 3.332616 \\
\hline $\mathrm{O}$ & 3.735128 & -19.693678 & 4.887188 \\
\hline $\mathrm{Zn}$ & 5.385652 & -19.269771 & 3.841314 \\
\hline $\mathrm{O}$ & 5.958252 & -17.482053 & 3.394033 \\
\hline $\mathrm{Zn}$ & 6.464248 & -16.988255 & 1.570225 \\
\hline $\mathrm{Zn}$ & 7.302249 & -14.954217 & -0.847407 \\
\hline $\mathrm{O}$ & 6.960460 & -15.174722 & 1.050732 \\
\hline $\mathrm{Zn}$ & 5.906874 & -13.727776 & 1.892427 \\
\hline $\mathrm{O}$ & 5.078184 & -12.323426 & 0.812872 \\
\hline $\mathrm{Zn}$ & 5.075392 & -12.507940 & -1.136289 \\
\hline $\mathrm{O}$ & 3.477458 & -12.182540 & -2.207168 \\
\hline $\mathrm{Zn}$ & 3.013410 & -1.214048 & 2.032814 \\
\hline $\mathrm{O}$ & 3.835107 & -2.671257 & 3.056422 \\
\hline $\mathrm{Zn}$ & 3.161704 & -3.053792 & 4.852024 \\
\hline $\mathrm{O}$ & 2.357828 & -1.651114 & 5.949090 \\
\hline $\mathrm{Zn}$ & 0.576658 & -2.108121 & 6.744836 \\
\hline $\mathrm{O}$ & -0.086630 & -3.863095 & 7.179480 \\
\hline $\mathrm{Zn}$ & -1.871735 & -4.332041 & 6.504168 \\
\hline $\mathrm{O}$ & -2.668459 & -3.044361 & 5.236193 \\
\hline $\mathrm{Zn}$ & 4.665380 & -4.151134 & 2.075142 \\
\hline $\mathrm{O}$ & 5.583534 & -3.841241 & 0.393073 \\
\hline
\end{tabular}




\begin{tabular}{|c|c|c|c|}
\hline $\mathrm{O}$ & 4.167954 & -5.974067 & 2.568026 \\
\hline $\mathrm{Zn}$ & 3.668314 & -6.268097 & 4.425024 \\
\hline $\mathrm{O}$ & 2.672241 & -4.877474 & 5.370371 \\
\hline $\mathrm{Zn}$ & 1.144616 & -5.236407 & 6.526087 \\
\hline $\mathrm{O}$ & 0.464576 & -7.071658 & 6.536995 \\
\hline $\mathrm{Zn}$ & 1.834972 & -8.267423 & 5.909078 \\
\hline $\mathrm{O}$ & 3.665337 & -8.064915 & 5.176821 \\
\hline $\mathrm{Zn}$ & 3.192260 & -9.442620 & 3.813270 \\
\hline $\mathrm{O}$ & 3.471357 & -11.436556 & 3.573485 \\
\hline $\mathrm{Zn}$ & 4.149609 & -13.000588 & 4.569630 \\
\hline $\mathrm{O}$ & 5.389138 & -14.216236 & 3.725574 \\
\hline $\mathrm{Zn}$ & 4.900889 & -16.046411 & 4.210214 \\
\hline $\mathrm{O}$ & 3.114676 & -16.429295 & 4.899913 \\
\hline $\mathrm{Zn}$ & 1.846014 & -14.983594 & 5.263042 \\
\hline $\mathrm{O}$ & 2.511277 & -13.206846 & 5.664760 \\
\hline $\mathrm{Zn}$ & 4.153309 & -7.432920 & 1.265861 \\
\hline $\mathrm{Zn}$ & 1.542804 & -9.405901 & 0.897562 \\
\hline $\mathrm{Zn}$ & 3.617845 & -11.241814 & 1.582802 \\
\hline $\mathrm{O}$ & 1.806120 & -11.543586 & 0.670654 \\
\hline $\mathrm{Zn}$ & 0.044064 & -11.681100 & 1.595459 \\
\hline $\mathrm{O}$ & -1.735813 & -11.953069 & 0.674723 \\
\hline $\mathrm{Zn}$ & -2.588910 & -13.643833 & 1.214238 \\
\hline $\mathrm{O}$ & -2.716744 & -14.103188 & 3.107803 \\
\hline $\mathrm{O}$ & 3.325199 & -9.133430 & 1.816468 \\
\hline $\mathrm{O}$ & -0.218289 & -9.542195 & 1.824522 \\
\hline $\mathrm{O}$ & -0.355607 & -0.592540 & 5.834119 \\
\hline $\mathrm{O}$ & -4.430277 & -2.821782 & 2.314568 \\
\hline $\mathrm{O}$ & -5.461550 & -4.824430 & 4.808903 \\
\hline $\mathrm{Zn}$ & 2.421062 & -18.259010 & 4.927220 \\
\hline $\mathrm{O}$ & 5.048051 & -20.724588 & 2.491399 \\
\hline $\mathrm{Zn}$ & 4.942162 & -19.872568 & 0.735837 \\
\hline $\mathrm{O}$ & 5.974896 & -18.306240 & 0.198637 \\
\hline $\mathrm{Zn}$ & 5.791272 & -17.695179 & -1.648217 \\
\hline $\mathrm{Zn}$ & 5.926586 & -14.872078 & -3.176291 \\
\hline $\mathrm{O}$ & 6.579170 & -13.446518 & -1.951001 \\
\hline $\mathrm{O}$ & 7.002692 & -16.307407 & -2.297861 \\
\hline $\mathrm{O}$ & 1.659509 & -17.239898 & -4.682982 \\
\hline $\mathrm{Zn}$ & 1.026142 & -19.002304 & -4.234732 \\
\hline $\mathrm{O}$ & -0.798474 & -19.397893 & -3.493813 \\
\hline $\mathrm{O}$ & 1.876990 & -20.539343 & -3.292437 \\
\hline $\mathrm{O}$ & -2.291501 & -18.404697 & -0.591475 \\
\hline $\mathrm{Zn}$ & -3.118268 & -16.926524 & 0.395949 \\
\hline $\mathrm{Zn}$ & -2.980689 & -18.578467 & 3.093566 \\
\hline $\mathrm{O}$ & -1.726105 & -19.881378 & 2.403058 \\
\hline $\mathrm{Zn}$ & -2.101153 & -14.801113 & -1.939161 \\
\hline
\end{tabular}




$\begin{array}{crrr}\mathrm{O} & -2.616591 & -15.101890 & -0.088414 \\ \mathrm{O} & -4.041147 & -17.228285 & 2.075826 \\ \mathrm{O} & -1.567876 & -4.679967 & -2.384562 \\ \mathrm{Zn} & -3.353299 & -5.072937 & -1.698954 \\ \mathrm{O} & -3.836499 & -6.906723 & -1.221453 \\ \mathrm{Zn} & -4.350831 & -7.393060 & 0.613826 \\ \mathrm{O} & 4.157832 & -3.729880 & -2.126214 \\ \mathrm{O} & -0.069249 & -15.184089 & 4.889933 \\ \mathrm{O} & -2.623491 & -17.320835 & 4.600663\end{array}$

$(\mathrm{ZnO})_{12}-\mathrm{CH}_{3} \mathrm{CH}_{2} \mathrm{OH}$ (binding via $\mathrm{O}$ (alcohol) $-\mathrm{Zn}(\mathrm{ZnO}$ cluster) bond)

\begin{tabular}{|c|c|c|c|}
\hline $\mathrm{C}$ & 4.063187 & 1.286644 & 4.304290 \\
\hline $\mathrm{C}$ & 4.486815 & 2.489603 & 3.492876 \\
\hline $\mathrm{O}$ & 4.609527 & 2.073405 & 2.114395 \\
\hline $\mathrm{Zn}$ & 2.763014 & 1.248688 & 1.222417 \\
\hline $\mathrm{O}$ & 2.950470 & -0.699152 & 1.199807 \\
\hline $\mathrm{Zn}$ & 1.477558 & -1.772631 & 2.001874 \\
\hline $\mathrm{O}$ & -0.036843 & -1.200051 & 3.053119 \\
\hline $\mathrm{Zn}$ & -0.181966 & 0.797231 & 2.821939 \\
\hline $\mathrm{O}$ & -2.102644 & 0.698861 & 2.248248 \\
\hline $\mathrm{Zn}$ & -2.364794 & 1.641550 & 0.579083 \\
\hline $\mathrm{O}$ & -1.113650 & 2.908876 & -0.356118 \\
\hline $\mathrm{Zn}$ & 0.682255 & 2.844590 & 0.364358 \\
\hline $\mathrm{O}$ & 1.140590 & 2.043048 & 2.158966 \\
\hline $\mathrm{Zn}$ & -1.249023 & 1.612681 & -1.900037 \\
\hline $\mathrm{Zn}$ & -2.365834 & -1.252215 & -1.059291 \\
\hline $\mathrm{O}$ & -2.804197 & 0.625841 & -1.111510 \\
\hline $\mathrm{Zn}$ & 0.505457 & -0.974684 & -2.752727 \\
\hline $\mathrm{Zn}$ & 2.534095 & -1.684771 & -0.503063 \\
\hline $\mathrm{Zn}$ & -0.442628 & -3.019027 & -0.283731 \\
\hline $\mathrm{O}$ & -2.155763 & -2.410527 & 0.570708 \\
\hline $\mathrm{Zn}$ & -1.821683 & -1.282442 & 2.113718 \\
\hline $\mathrm{O}$ & -0.873965 & -2.142363 & -2.057546 \\
\hline $\mathrm{O}$ & 2.442973 & -0.866342 & -2.262235 \\
\hline $\mathrm{Zn}$ & 2.095196 & 1.107859 & -2.021020 \\
\hline $\mathrm{O}$ & 2.400123 & 2.251628 & -0.490236 \\
\hline $\mathrm{O}$ & 0.282119 & 1.024188 & -2.914347 \\
\hline $\mathrm{O}$ & 1.361248 & -3.024861 & 0.420935 \\
\hline $\mathrm{H}$ & 4.805142 & 0.489389 & 4.225448 \\
\hline $\mathrm{H}$ & 3.963658 & 1.569162 & 5.354529 \\
\hline $\mathrm{H}$ & 3.098603 & 0.907821 & 3.957634 \\
\hline $\mathrm{H}$ & 3.746512 & 3.289677 & 3.560223 \\
\hline $\mathrm{H}$ & 5.454194 & 2.867191 & 3.828537 \\
\hline
\end{tabular}


$(\mathrm{ZnO})_{12}-\mathrm{CH}_{3} \mathrm{CH}_{2} \mathrm{OH}$ (binding via $\mathrm{H}($ alcohol $)-\mathrm{O}(\mathrm{ZnO}$ cluster) bond)

\begin{tabular}{|c|c|c|c|}
\hline $\mathrm{C}$ & 0.232819 & -0.537183 & -0.066177 \\
\hline $\mathrm{C}$ & 0.197545 & -0.655241 & 1.446324 \\
\hline $\mathrm{O}$ & 1.347988 & -0.045591 & 2.006376 \\
\hline $\mathrm{O}$ & 1.178989 & -0.449344 & 4.439673 \\
\hline $\mathrm{Ti}$ & 1.586092 & 1.051749 & 5.755853 \\
\hline $\mathrm{O}$ & -0.346954 & 1.294748 & 6.186118 \\
\hline $\mathrm{Ti}$ & -0.647057 & 1.120954 & 8.101229 \\
\hline $\mathrm{O}$ & 0.780969 & 1.297160 & 9.509126 \\
\hline $\mathrm{Ti}$ & 2.579028 & 1.088675 & 8.816119 \\
\hline $\mathrm{O}$ & 3.833533 & -0.446947 & 9.136059 \\
\hline $\mathrm{Ti}$ & 4.077275 & -0.487414 & 7.143496 \\
\hline $\mathrm{O}$ & 3.845445 & -2.064940 & 6.047928 \\
\hline $\mathrm{Ti}$ & 2.218987 & -2.040146 & 4.868965 \\
\hline $\mathrm{O}$ & 1.505576 & -3.762542 & 5.587527 \\
\hline $\mathrm{Ti}$ & 2.931352 & -3.565013 & 7.005044 \\
\hline $\mathrm{O}$ & 2.611161 & -3.736727 & 8.916786 \\
\hline $\mathrm{Ti}$ & 2.934703 & -1.997812 & 9.873019 \\
\hline $\mathrm{O}$ & 1.093409 & -1.991052 & 10.682348 \\
\hline $\mathrm{Ti}$ & 0.051754 & -0.433123 & 10.214622 \\
\hline $\mathrm{O}$ & -1.576087 & -0.385259 & 9.030115 \\
\hline $\mathrm{Ti}$ & -1.802037 & -1.959944 & 7.927917 \\
\hline $\mathrm{Ti}$ & 0.680433 & -3.467828 & 9.369187 \\
\hline $\mathrm{O}$ & -0.853999 & -3.715811 & 8.220639 \\
\hline $\mathrm{Ti}$ & -0.284807 & -3.579869 & 6.301410 \\
\hline $\mathrm{O}$ & -1.522658 & -2.040475 & 5.933851 \\
\hline $\mathrm{Ti}$ & -0.685100 & -0.436727 & 5.251056 \\
\hline $\mathrm{O}$ & 3.128921 & 1.274015 & 6.888892 \\
\hline $\mathrm{H}$ & 1.118336 & -1.037674 & -0.465985 \\
\hline $\mathrm{H}$ & -0.654108 & -0.994964 & -0.511320 \\
\hline $\mathrm{H}$ & 0.266154 & 0.513721 & -0.364429 \\
\hline $\mathrm{H}$ & -0.713761 & -0.175423 & 1.830550 \\
\hline $\mathrm{H}$ & 0.150800 & -1.715491 & 1.732120 \\
\hline $\mathrm{H}$ & 1.297549 & -0.193383 & 3.055336 \\
\hline
\end{tabular}

$(\mathrm{ZnO})_{12}-\mathrm{CH}_{3}\left(\mathrm{CH}_{2}\right)_{2} \mathrm{OH}$ (binding via $\mathrm{O}($ alcohol $)-\mathrm{Zn}(\mathrm{ZnO}$ cluster) bond) 


\begin{tabular}{|c|c|c|c|}
\hline $\mathrm{C}$ & 0.079057 & -0.167196 & 0.101924 \\
\hline $\mathrm{C}$ & -0.152914 & 0.103342 & 1.573287 \\
\hline $\mathrm{O}$ & 1.124495 & 0.379991 & 2.185391 \\
\hline $\mathrm{Ti}$ & 2.414619 & 1.823949 & 1.176966 \\
\hline $\mathrm{O}$ & 3.476852 & 1.222320 & -0.353388 \\
\hline $\mathrm{Ti}$ & 3.462493 & 2.351745 & -2.005494 \\
\hline $\mathrm{O}$ & 2.334330 & 3.876428 & -2.355436 \\
\hline $\mathrm{Ti}$ & 1.524508 & 4.524477 & -0.635686 \\
\hline $\mathrm{O}$ & 2.132249 & 6.416167 & -0.938934 \\
\hline $\mathrm{Ti}$ & 3.173628 & 6.984044 & 0.592852 \\
\hline $\mathrm{O}$ & 3.145838 & 6.103520 & 2.397875 \\
\hline $\mathrm{Ti}$ & 2.408147 & 4.306325 & 2.389932 \\
\hline $\mathrm{O}$ & 1.254489 & 3.488658 & 0.965095 \\
\hline $\mathrm{Ti}$ & 5.142716 & 6.020645 & 2.210407 \\
\hline $\mathrm{Ti}$ & 6.021660 & 6.400241 & -0.869987 \\
\hline $\mathrm{O}$ & 5.170725 & 7.265085 & 0.628999 \\
\hline $\mathrm{Ti}$ & 7.080885 & 3.836822 & 0.804500 \\
\hline $\mathrm{Ti}$ & 5.467815 & 1.428983 & -0.399445 \\
\hline $\mathrm{Ti}$ & 6.202761 & 4.059210 & -2.251577 \\
\hline $\mathrm{O}$ & 5.198954 & 5.766048 & -2.588059 \\
\hline $\mathrm{Ti}$ & 3.264197 & 5.663895 & -2.417511 \\
\hline $\mathrm{O}$ & 7.332097 & 4.873764 & -0.798043 \\
\hline $\mathrm{O}$ & 6.533239 & 1.942833 & 1.138318 \\
\hline $\mathrm{Ti}$ & 5.350431 & 2.695332 & 2.575619 \\
\hline $\mathrm{O}$ & 3.420907 & 2.620197 & 2.757492 \\
\hline $\mathrm{O}$ & 6.271659 & 4.491743 & 2.521052 \\
\hline $\mathrm{O}$ & 5.460187 & 2.269214 & -2.226372 \\
\hline $\mathrm{H}$ & 0.802029 & -0.982006 & 0.002737 \\
\hline $\mathrm{C}$ & -1.226032 & -0.528608 & -0.603022 \\
\hline $\mathrm{H}$ & 0.525613 & 0.723775 & -0.352237 \\
\hline $\mathrm{H}$ & -0.812101 & 0.964240 & 1.710855 \\
\hline $\mathrm{H}$ & -0.593083 & -0.767710 & 2.064975 \\
\hline $\mathrm{H}$ & 0.999109 & 0.634282 & 3.108817 \\
\hline $\mathrm{H}$ & -1.053705 & -0.716557 & -1.664071 \\
\hline $\mathrm{H}$ & -1.667331 & -1.429658 & -0.169192 \\
\hline $\mathrm{H}$ & -1.955547 & 0.280833 & -0.517998 \\
\hline
\end{tabular}

$(\mathrm{ZnO})_{12}-\mathrm{CH}_{3}\left(\mathrm{CH}_{2}\right)_{2} \mathrm{OH}$ (binding via $\mathrm{H}($ alcohol $)-\mathrm{O}(\mathrm{ZnO}$ cluster) bond $)$
C
-1.134898
0.186841
$-0.283762$
$-0.850861$
C
$-0.247481$
$-0.086610$
C
$-0.027161$
$-0.249987$
1.418053
$\mathrm{O}$
1.216692
$-0.182534$
2.091855
$\mathrm{O}$
0.774115
$-0.555052$
4.501349 


\begin{tabular}{|c|c|c|c|}
\hline $\mathrm{Zn}$ & 2.068286 & 0.421527 & 5.734010 \\
\hline $\mathrm{O}$ & 0.611396 & 1.536392 & 6.517323 \\
\hline $\mathrm{Zn}$ & 0.554552 & 1.389833 & 8.449750 \\
\hline $\mathrm{O}$ & 1.998866 & 0.573544 & 9.581881 \\
\hline $\mathrm{Zn}$ & 3.309639 & -0.449687 & 8.587008 \\
\hline $\mathrm{O}$ & 3.497356 & -2.449048 & 8.710276 \\
\hline $\mathrm{Zn}$ & 3.317039 & -2.443244 & 6.710801 \\
\hline $\mathrm{O}$ & 3.564241 & -0.445986 & 6.586793 \\
\hline $\mathrm{Zn}$ & -0.688424 & 0.323650 & 5.591735 \\
\hline $\mathrm{Zn}$ & 0.770838 & -2.497067 & 4.716022 \\
\hline $\mathrm{O}$ & 2.226661 & -3.568943 & 5.590810 \\
\hline $\mathrm{Zn}$ & 0.751476 & -4.415317 & 6.671054 \\
\hline $\mathrm{O}$ & -0.692421 & -3.597830 & 5.532907 \\
\hline $\mathrm{Zn}$ & -2.005385 & -2.581902 & 6.531307 \\
\hline $\mathrm{Zn}$ & -2.006950 & -0.583896 & 8.401115 \\
\hline $\mathrm{Zn}$ & 0.536295 & -0.535271 & 10.403556 \\
\hline $\mathrm{Zn}$ & 1.982071 & -3.324206 & 9.531970 \\
\hline $\mathrm{O}$ & 0.686342 & -4.552983 & 8.600590 \\
\hline $\mathrm{Zn}$ & -0.753450 & -3.415028 & 9.408518 \\
\hline $\mathrm{O}$ & 0.536844 & -2.461943 & 10.622719 \\
\hline $\mathrm{O}$ & -2.189939 & -0.581724 & 6.399477 \\
\hline $\mathrm{O}$ & -2.258972 & -2.579629 & 8.528448 \\
\hline $\mathrm{O}$ & -0.921918 & 0.543314 & 9.525242 \\
\hline $\mathrm{H}$ & 0.801903 & -1.112270 & -0.354792 \\
\hline $\mathrm{H}$ & 0.753175 & 0.649254 & -0.357035 \\
\hline $\mathrm{H}$ & -0.662442 & 0.604761 & 1.693665 \\
\hline $\mathrm{H}$ & -0.570171 & -1.162865 & 1.705017 \\
\hline $\mathrm{H}$ & 1.035460 & -0.320264 & 3.123148 \\
\hline $\mathrm{H}$ & -0.967225 & -0.287522 & -1.929838 \\
\hline $\mathrm{H}$ & -1.709503 & -1.179295 & -0.598406 \\
\hline $\mathrm{H}$ & -1.749321 & 0.588300 & -0.610536 \\
\hline
\end{tabular}

$(\mathrm{ZnO})_{12}-\mathrm{CH}_{3}\left(\mathrm{CH}_{2}\right)_{3} \mathrm{OH}$ (binding via $\mathrm{O}($ alcohol $)-\mathrm{Zn}(\mathrm{ZnO}$ cluster) bond)

$\begin{array}{crrr}\mathrm{C} & -1.311369 & -0.389165 & -2.070383 \\ \mathrm{C} & -1.384593 & -0.267553 & -0.550183 \\ \mathrm{C} & -0.003471 & -0.061642 & 0.069233 \\ \mathrm{C} & -0.077788 & 0.065871 & 1.575068 \\ \mathrm{O} & 1.267826 & 0.228962 & 2.073347 \\ \mathrm{Zn} & 2.403794 & 1.865521 & 1.211553 \\ \mathrm{O} & 3.420315 & 2.672899 & 2.779048 \\ \mathrm{Zn} & 5.349492 & 2.745991 & 2.595066 \\ \mathrm{O} & 6.291593 & 4.532228 & 2.554642 \\ \mathrm{Zn} & 7.167096 & 3.866312 & 0.877865\end{array}$




\begin{tabular}{|c|c|c|c|}
\hline $\mathrm{O}$ & 6.520320 & 1.984040 & 1.157114 \\
\hline $\mathrm{Zn}$ & 5.457300 & 1.449515 & -0.373875 \\
\hline $\mathrm{O}$ & 5.446309 & 2.258305 & -2.212566 \\
\hline $\mathrm{Zn}$ & 6.192392 & 4.043608 & -2.239651 \\
\hline $\mathrm{O}$ & 7.312284 & 4.848110 & -0.779491 \\
\hline $\mathrm{Zn}$ & 6.026307 & 6.390511 & -0.861660 \\
\hline $\mathrm{Zn}$ & 3.267847 & 5.659454 & -2.411052 \\
\hline $\mathrm{O}$ & 5.201822 & 5.757947 & -2.577209 \\
\hline $\mathrm{O}$ & 3.467544 & 1.253499 & -0.319101 \\
\hline $\mathrm{Zn}$ & 3.449603 & 2.350974 & -1.987201 \\
\hline $\mathrm{Zn}$ & 1.519026 & 4.536173 & -0.627666 \\
\hline $\mathrm{O}$ & 1.248027 & 3.523815 & 0.988520 \\
\hline $\mathrm{Zn}$ & 2.401052 & 4.352507 & 2.406602 \\
\hline $\mathrm{Zn}$ & 5.153598 & 6.054226 & 2.236342 \\
\hline $\mathrm{O}$ & 5.186717 & 7.271382 & 0.633232 \\
\hline $\mathrm{Zn}$ & 3.189079 & 7.004567 & 0.599369 \\
\hline $\mathrm{O}$ & 3.158585 & 6.140311 & 2.411383 \\
\hline $\mathrm{O}$ & 2.327929 & 3.878687 & -2.341785 \\
\hline $\mathrm{O}$ & 2.150065 & 6.423357 & -0.928429 \\
\hline $\mathrm{H}$ & 0.649156 & -0.903357 & -0.184268 \\
\hline $\mathrm{H}$ & 0.457501 & 0.844471 & -0.341364 \\
\hline $\mathrm{H}$ & -0.675655 & 0.933547 & 1.864703 \\
\hline $\mathrm{H}$ & -0.507773 & -0.832914 & 2.022903 \\
\hline $\mathrm{H}$ & 1.252042 & 0.295270 & 3.037085 \\
\hline $\mathrm{H}$ & -1.838692 & -1.170943 & -0.130286 \\
\hline $\mathrm{H}$ & -2.033127 & 0.571357 & -0.278188 \\
\hline $\mathrm{H}$ & -2.302012 & -0.539394 & -2.505238 \\
\hline $\mathrm{H}$ & -0.883073 & 0.514608 & -2.512173 \\
\hline $\mathrm{H}$ & -0.683688 & -1.235676 & -2.361726 \\
\hline
\end{tabular}

$(\mathrm{ZnO})_{12}-\mathrm{CH}_{3}\left(\mathrm{CH}_{2}\right)_{3} \mathrm{OH}$ (binding via $\mathrm{H}($ alcohol $)-\mathrm{O}(\mathrm{ZnO}$ cluster) bond)

$\begin{array}{crrr}\mathrm{C} & -1.090904 & 0.151060 & -2.281529 \\ \mathrm{C} & -1.270477 & 0.139995 & -0.764536 \\ \mathrm{C} & 0.063828 & 0.041340 & -0.027568 \\ \mathrm{C} & -0.097941 & 0.010311 & 1.483317 \\ \mathrm{O} & 1.171528 & -0.057368 & 2.108913 \\ \mathrm{O} & 0.789013 & -0.551315 & 4.508740 \\ \mathrm{Zn} & 2.083840 & 0.427532 & 5.738114 \\ \mathrm{O} & 0.625642 & 1.534159 & 6.527566 \\ \mathrm{Zn} & 0.570071 & 1.385513 & 8.459325 \\ \mathrm{O} & 2.014597 & 0.558131 & 9.581646 \\ \mathrm{Zn} & 3.328476 & -0.460334 & 8.587884 \\ \mathrm{O} & 3.513443 & -2.459544 & 8.699499\end{array}$




$\begin{array}{crrr}\mathrm{Zn} & 3.342826 & -2.447860 & 6.699627 \\ \mathrm{O} & 3.575339 & -0.449110 & 6.587960 \\ \mathrm{Zn} & -0.674200 & 0.326682 & 5.597573 \\ \mathrm{Zn} & -1.997217 & -0.590483 & 8.410257 \\ \mathrm{Zn} & 0.552026 & -0.546321 & 10.408047 \\ \mathrm{Zn} & 2.003053 & -3.336017 & 9.526848 \\ \mathrm{O} & 0.707619 & -4.557430 & 8.587860 \\ \mathrm{Zn} & 0.772630 & -4.418440 & 6.658778 \\ \mathrm{O} & 2.245375 & -3.567086 & 5.581409 \\ \mathrm{Zn} & 0.791205 & -2.493207 & 4.707565 \\ \mathrm{O} & -0.671431 & -3.590437 & 5.529746 \\ \mathrm{Zn} & -1.988510 & -2.582016 & 6.527793 \\ \mathrm{Zn} & -0.733798 & -3.428900 & 9.403146 \\ \mathrm{O} & 0.556309 & -2.473307 & 10.613930 \\ \mathrm{O} & -2.171210 & -0.581850 & 6.407964 \\ \mathrm{O} & -2.235768 & -2.587790 & 8.524445 \\ \mathrm{O} & -0.905050 & 0.532893 & 9.530135 \\ \mathrm{H} & 0.593663 & -0.864739 & -0.342540 \\ \mathrm{H} & 0.697004 & 0.894634 & -0.295773 \\ \mathrm{H} & -0.640397 & 0.910168 & 1.809347 \\ \mathrm{H} & -0.710442 & -0.858348 & 1.765872 \\ \mathrm{H} & 1.017758 & -0.250540 & 3.133642 \\ \mathrm{H} & -1.906905 & -0.703904 & -0.477540 \\ \mathrm{H} & -1.792555 & 1.050052 & -0.450766 \\ \mathrm{H} & -2.051486 & 0.222873 & -2.797201 \\ \mathrm{H} & -0.477479 & 1.000734 & -2.593741 \\ \mathrm{H} & -0.593767 & -0.762286 & -619761\end{array}$

$(\mathrm{ZnO})_{12}-\mathrm{CH}_{3}\left(\mathrm{CH}_{2}\right)_{4} \mathrm{OH}$ (binding via $\mathrm{O}$ (alcohol) $\mathrm{Zn}(\mathrm{ZnO}$ cluster) bond)

$\begin{array}{crrr}\mathrm{C} & -0.048949 & 0.079204 & 0.074022 \\ \mathrm{C} & -0.070409 & 0.105813 & 1.587526 \\ \mathrm{O} & 1.290460 & 0.237143 & 2.054374 \\ \mathrm{Ti} & 2.445152 & 1.866509 & 1.213021 \\ \mathrm{O} & 3.505152 & 1.254675 & -0.318275 \\ \mathrm{Ti} & 3.492183 & 2.352074 & -1.984610 \\ \mathrm{O} & 2.378293 & 3.885805 & -2.333169 \\ \mathrm{Ti} & 1.562526 & 4.539334 & -0.621776 \\ \mathrm{O} & 2.205614 & 6.423708 & -0.911304 \\ \mathrm{Ti} & 3.240614 & 7.002964 & 0.618692 \\ \mathrm{O} & 3.209396 & 6.136297 & 2.428770 \\ \mathrm{Ti} & 2.449632 & 4.349574 & 2.424151 \\ \mathrm{O} & 1.299322 & 3.533601 & 0.998571 \\ \mathrm{Ti} & 5.203167 & 6.051302 & 2.256911\end{array}$




$\begin{array}{lrrr}\mathrm{Ti} & 6.083999 & 6.392243 & -0.847104 \\ \mathrm{O} & 5.238550 & 7.262043 & 0.649586 \\ \mathrm{Ti} & 7.216452 & 3.859064 & 0.899281 \\ \mathrm{Ti} & 5.495202 & 1.447572 & -0.365314 \\ \mathrm{Ti} & 6.235333 & 4.043678 & -2.229521 \\ \mathrm{O} & 5.252807 & 5.763414 & -2.560124 \\ \mathrm{Ti} & 3.318945 & 5.665782 & -2.399997 \\ \mathrm{O} & 7.352263 & 4.836627 & -0.760984 \\ \mathrm{O} & 6.556038 & 1.980636 & 1.166910 \\ \mathrm{Ti} & 5.390729 & 2.739851 & 2.610042 \\ \mathrm{O} & 3.462042 & 2.664883 & 2.785877 \\ \mathrm{O} & 6.334503 & 4.524783 & 2.571464 \\ \mathrm{O} & 5.488128 & 2.259644 & -2.202505 \\ \mathrm{H} & 0.632176 & -0.711896 & -0.255690 \\ \mathrm{C} & -1.442866 & -0.159383 & -0.504368 \\ \mathrm{H} & 0.347794 & 1.032051 & -0.295141 \\ \mathrm{H} & -0.658937 & 0.950158 & 1.954996 \\ \mathrm{H} & -0.484966 & -0.822617 & 1.986665 \\ \mathrm{H} & 1.300995 & 0.265857 & 3.019920 \\ \mathrm{C} & -1.442681 & -0.180963 & -2.032185 \\ \mathrm{H} & -1.832903 & -1.113129 & -0.130758 \\ \mathrm{H} & -2.125585 & 0.622857 & -0.153264 \\ \mathrm{C} & -2.827739 & -0.465733 & -2.608812 \\ \mathrm{H} & -1.077514 & 0.781730 & -2.404925 \\ \mathrm{H} & -0.736338 & -0.942420 & -2.379225 \\ \mathrm{H} & -2.811449 & -0.471443 & -3.701273 \\ \mathrm{H} & -3.195483 & -1.439506 & -2.273272 \\ & & & \end{array}$

$(\mathrm{ZnO})_{12}-\mathrm{CH}_{3}\left(\mathrm{CH}_{2}\right)_{4} \mathrm{OH}$ (binding via $\mathrm{H}($ alcohol $)-\mathrm{O}(\mathrm{ZnO}$ cluster $)$ bond $)$

$\begin{array}{crrr}\mathrm{C} & -2.377715 & 0.201012 & -3.072530 \\ \mathrm{C} & -1.097361 & 0.376291 & -2.258710 \\ \mathrm{C} & -1.301380 & 0.071101 & -0.775071 \\ \mathrm{C} & -0.030882 & 0.272240 & 0.048859 \\ \mathrm{C} & -0.228276 & -0.046147 & 1.521548 \\ \mathrm{O} & 0.973398 & 0.188142 & 2.234725 \\ \mathrm{O} & 0.626858 & -0.551081 & 4.578836 \\ \mathrm{Zn} & 2.097846 & 0.309687 & 5.683103 \\ \mathrm{O} & 0.801851 & 1.526871 & 6.603634 \\ \mathrm{Zn} & 0.830057 & 1.367940 & 8.533665 \\ \mathrm{O} & 2.280698 & 0.490518 & 9.613879 \\ \mathrm{Zn} & 3.368974 & -0.644771 & 8.500611 \\ \mathrm{O} & 3.522001 & -2.645479 & 8.590317 \\ \mathrm{Zn} & 3.326426 & -2.614278 & 6.590941\end{array}$




$\begin{array}{crrr}\mathrm{O} & 3.579842 & -0.617598 & 6.498461 \\ \mathrm{Zn} & -0.660573 & 0.445804 & 5.791577 \\ \mathrm{Zn} & -1.952877 & -0.440033 & 8.633051 \\ \mathrm{O} & -0.636921 & 0.558029 & 9.640835 \\ \mathrm{Zn} & 0.800632 & -0.565368 & 10.480635 \\ \mathrm{O} & 0.743010 & -2.492756 & 10.667366 \\ \mathrm{Zn} & 2.016625 & -3.480368 & 9.466943 \\ \mathrm{Zn} & 0.543027 & -4.418221 & 6.709919 \\ \mathrm{O} & 2.002222 & -3.608989 & 5.590173 \\ \mathrm{Zn} & 0.562681 & -2.492270 & 4.759258 \\ \mathrm{O} & -0.912503 & -3.544171 & 5.627221 \\ \mathrm{Zn} & -2.008282 & -2.432795 & 6.755854 \\ \mathrm{Zn} & -0.719263 & -3.332506 & 9.582374 \\ \mathrm{O} & 0.556785 & -4.571709 & 8.636774 \\ \mathrm{O} & -2.155800 & -0.432433 & 6.632475 \\ \mathrm{O} & -2.217971 & -2.432737 & 8.754804 \\ \mathrm{H} & 0.771015 & -0.363804 & -0.342497 \\ \mathrm{H} & 0.306995 & 1.310672 & -0.041140 \\ \mathrm{H} & -1.041539 & 0.576828 & 1.922438 \\ \mathrm{H} & -0.540597 & -1.095454 & 1.629242 \\ \mathrm{H} & 0.826058 & -0.117960 & 3.230810 \\ \mathrm{H} & -1.650463 & -0.962664 & -0.665564 \\ \mathrm{H} & -2.096821 & 0.715780 & -0.382450 \\ \mathrm{H} & -0.731100 & 1.402769 & -2.366501 \\ \mathrm{H} & -0.316125 & -0.280063 & -2.656789 \\ \mathrm{H} & -2.211180 & -4.130422 \\ \mathrm{H} & -2.751214 & -0.823957 & -2.994393 \\ \mathrm{H} & -3.162778 & 0.871641 & \\ & & & \end{array}$

$(\mathrm{ZnO})_{12}-\mathrm{CH}_{3}\left(\mathrm{CH}_{2}\right)_{7} \mathrm{OH}$ (binding via $\mathrm{O}$ (alcohol) $-\mathrm{Zn}(\mathrm{ZnO}$ cluster) bond)

$\begin{array}{crrr}\mathrm{C} & -4.364847 & -0.232326 & -6.103929 \\ \mathrm{C} & -4.313438 & -0.229468 & -4.577415 \\ \mathrm{C} & -2.884536 & -0.166022 & -4.038033 \\ \mathrm{C} & -2.826019 & -0.154563 & -2.511482 \\ \mathrm{C} & -1.400845 & -0.085461 & -1.963670 \\ \mathrm{C} & -1.362388 & -0.056219 & -0.436596 \\ \mathrm{C} & 0.060189 & 0.012542 & 0.117757 \\ \mathrm{C} & 0.056867 & 0.073038 & 1.630328 \\ \mathrm{O} & 1.424224 & 0.192660 & 2.078807 \\ \mathrm{Zn} & 2.533290 & 1.857005 & 1.254996 \\ \mathrm{O} & 3.534583 & 2.649962 & 2.835951 \\ \mathrm{Zn} & 5.458979 & 2.752707 & 2.636691 \\ \mathrm{O} & 6.371358 & 4.549663 & 2.611217\end{array}$




\begin{tabular}{|c|c|c|c|}
\hline $\mathrm{Zn}$ & 7.271515 & 3.928073 & 0.934951 \\
\hline $\mathrm{O}$ & 6.622453 & 2.044259 & 1.169184 \\
\hline $\mathrm{Zn}$ & 5.568243 & 1.497948 & -0.360132 \\
\hline $\mathrm{O}$ & 5.536477 & 2.324500 & -2.186365 \\
\hline $\mathrm{Zn}$ & 6.277247 & 4.109373 & -2.210627 \\
\hline $\mathrm{O}$ & 7.384526 & 4.897285 & -0.730988 \\
\hline $\mathrm{Zn}$ & 6.105569 & 6.434477 & -0.795087 \\
\hline $\mathrm{Zn}$ & 3.317299 & 5.688668 & -2.328484 \\
\hline $\mathrm{O}$ & 5.249032 & 5.801942 & -2.494472 \\
\hline $\mathrm{O}$ & 3.584585 & 1.295684 & -0.293936 \\
\hline $\mathrm{Zn}$ & 3.542174 & 2.383287 & -1.963811 \\
\hline $\mathrm{Zn}$ & 1.536095 & 4.506259 & -0.590668 \\
\hline $\mathrm{O}$ & 1.383391 & 3.520339 & 1.058411 \\
\hline $\mathrm{Zn}$ & 2.502761 & 4.320942 & 2.511849 \\
\hline $\mathrm{Zn}$ & 5.221231 & 6.068514 & 2.330083 \\
\hline $\mathrm{O}$ & 5.199482 & 7.235562 & 0.698815 \\
\hline $\mathrm{Zn}$ & 3.209285 & 6.975155 & 0.710423 \\
\hline $\mathrm{O}$ & 3.228460 & 6.116349 & 2.519592 \\
\hline $\mathrm{O}$ & 2.399977 & 3.899504 & -2.291797 \\
\hline $\mathrm{O}$ & 2.188965 & 6.394949 & -0.826305 \\
\hline $\mathrm{H}$ & 0.637326 & -0.859602 & -0.205108 \\
\hline $\mathrm{H}$ & 0.566646 & 0.901595 & -0.275693 \\
\hline $\mathrm{H}$ & -0.511938 & 0.937265 & 1.982559 \\
\hline $\mathrm{H}$ & -0.370869 & -0.837503 & 2.055814 \\
\hline $\mathrm{H}$ & 1.453135 & 0.197688 & 3.044213 \\
\hline $\mathrm{H}$ & -1.863999 & -0.948909 & -0.045545 \\
\hline $\mathrm{H}$ & -1.931118 & 0.810011 & -0.079753 \\
\hline $\mathrm{H}$ & -0.903544 & 0.810115 & -2.354302 \\
\hline $\mathrm{H}$ & -0.828874 & -0.947961 & -2.325309 \\
\hline $\mathrm{H}$ & -3.318293 & -1.054737 & -2.124363 \\
\hline $\mathrm{H}$ & -3.397814 & 0.702473 & -2.135737 \\
\hline $\mathrm{H}$ & -2.391563 & 0.733232 & -4.426222 \\
\hline $\mathrm{H}$ & -2.316730 & -1.024331 & -4.417145 \\
\hline $\mathrm{H}$ & -4.802799 & -1.130511 & -4.192013 \\
\hline $\mathrm{H}$ & -4.880749 & 0.624992 & -4.192663 \\
\hline $\mathrm{H}$ & -5.393228 & -0.287401 & -6.468779 \\
\hline $\mathrm{H}$ & -3.912831 & 0.676547 & -6.510485 \\
\hline $\mathrm{H}$ & -3.818033 & -1.088015 & -6.509792 \\
\hline
\end{tabular}

$(\mathrm{ZnO})_{12}-\mathrm{CH}_{3}\left(\mathrm{CH}_{2}\right)_{7} \mathrm{OH}$ (binding via $\mathrm{H}($ alcohol $)-\mathrm{O}(\mathrm{ZnO}$ cluster) bond)
C
$-3.619123$
$-0.202462$
$-6.651470$
C
$-3.816269$
$-0.211005$
$-5.136914$
$\mathrm{C}$
$-2.492355$
$-0.164356$
$-4.375691$ 


\begin{tabular}{|c|c|c|c|}
\hline $\mathrm{C}$ & -2.671257 & -0.174724 & -2.858299 \\
\hline $\mathrm{C}$ & -1.343214 & -0.123584 & -2.104733 \\
\hline $\mathrm{C}$ & -1.510452 & -0.144375 & -0.585793 \\
\hline $\mathrm{C}$ & -0.171868 & -0.086639 & 0.147898 \\
\hline $\mathrm{C}$ & -0.321458 & -0.124406 & 1.659499 \\
\hline $\mathrm{O}$ & 0.953355 & -0.039571 & 2.272219 \\
\hline $\mathrm{O}$ & 0.682079 & -0.561447 & 4.694624 \\
\hline $\mathrm{Zn}$ & 2.150959 & 0.315327 & 5.780856 \\
\hline $\mathrm{O}$ & 0.849100 & 1.510360 & 6.713898 \\
\hline $\mathrm{Zn}$ & 0.918432 & 1.374573 & 8.642444 \\
\hline $\mathrm{O}$ & 2.374454 & 0.497032 & 9.707853 \\
\hline $\mathrm{Zn}$ & 3.475800 & -0.630354 & 8.603889 \\
\hline $\mathrm{O}$ & 3.665612 & -2.628708 & 8.692085 \\
\hline $\mathrm{Zn}$ & 3.443419 & -2.613565 & 6.696930 \\
\hline $\mathrm{O}$ & 3.620775 & -0.616372 & 6.604496 \\
\hline $\mathrm{Zn}$ & -0.610719 & 0.431397 & 5.898776 \\
\hline $\mathrm{Zn}$ & -1.866536 & -0.445442 & 8.757929 \\
\hline $\mathrm{O}$ & -0.537176 & 0.547858 & 9.748342 \\
\hline $\mathrm{Zn}$ & 0.908084 & -0.560207 & 10.589693 \\
\hline $\mathrm{O}$ & 0.891622 & -2.484368 & 10.777225 \\
\hline $\mathrm{Zn}$ & 2.175247 & -3.465826 & 9.588528 \\
\hline $\mathrm{Zn}$ & 0.659913 & -4.428364 & 6.828053 \\
\hline $\mathrm{O}$ & 2.109969 & -3.604890 & 5.710682 \\
\hline $\mathrm{Zn}$ & 0.667959 & -2.499974 & 4.878616 \\
\hline $\mathrm{O}$ & -0.797609 & -3.551282 & 5.757189 \\
\hline $\mathrm{Zn}$ & -1.906043 & -2.443663 & 6.871833 \\
\hline $\mathrm{Zn}$ & -0.564610 & -3.340307 & 9.703564 \\
\hline $\mathrm{O}$ & 0.719875 & -4.558139 & 8.753171 \\
\hline $\mathrm{O}$ & -2.092543 & -0.445042 & 6.759312 \\
\hline $\mathrm{O}$ & -2.053296 & -2.440934 & 8.867571 \\
\hline $\mathrm{H}$ & 0.456947 & -0.928676 & -0.161855 \\
\hline $\mathrm{H}$ & 0.360242 & 0.830915 & -0.126566 \\
\hline $\mathrm{H}$ & -0.961788 & 0.708971 & 1.983833 \\
\hline $\mathrm{H}$ & -0.827155 & -1.056047 & 1.953261 \\
\hline $\mathrm{H}$ & 0.840232 & -0.237761 & 3.290897 \\
\hline $\mathrm{H}$ & -2.049608 & -1.053611 & -0.294489 \\
\hline $\mathrm{H}$ & -2.133192 & 0.704133 & -0.278879 \\
\hline $\mathrm{H}$ & -0.798104 & 0.782497 & -2.394691 \\
\hline $\mathrm{H}$ & -0.722425 & -0.974310 & -2.409789 \\
\hline $\mathrm{H}$ & -3.219947 & -1.077703 & -2.564830 \\
\hline $\mathrm{H}$ & -3.289973 & 0.680268 & -2.560590 \\
\hline $\mathrm{H}$ & -1.939950 & 0.736531 & -4.669070 \\
\hline $\mathrm{H}$ & -1.875689 & -1.021056 & -4.673228 \\
\hline $\mathrm{H}$ & -4.368667 & -1.110224 & -4.843510 \\
\hline $\mathrm{H}$ & -4.431254 & 0.646003 & -4.841736 \\
\hline
\end{tabular}


H

$\mathrm{H}$

$\mathrm{H}$

\section{$(\mathrm{ZnO})_{36}-\mathrm{CH}_{3} \mathrm{CH}_{2} \mathrm{OH}$}

\begin{tabular}{|c|c|c|c|}
\hline $\mathrm{C}$ & -0.188543 & -0.030035 & 0.130693 \\
\hline $\mathrm{C}$ & -0.078792 & -0.037421 & 1.642528 \\
\hline $\mathrm{O}$ & 1.291594 & 0.020221 & 2.024736 \\
\hline $\mathrm{Zn}$ & 2.426406 & -1.836113 & 1.767443 \\
\hline $\mathrm{O}$ & 4.134348 & -1.185761 & 2.569408 \\
\hline $\mathrm{Zn}$ & 5.657233 & -2.395173 & 2.859273 \\
\hline $\mathrm{Zn}$ & 4.207931 & -4.107295 & 0.731633 \\
\hline $\mathrm{O}$ & 2.843967 & -2.847196 & 0.092810 \\
\hline $\mathrm{Zn}$ & 1.313274 & -3.897081 & -0.445320 \\
\hline $\mathrm{Zn}$ & 2.163878 & -6.834325 & 0.308348 \\
\hline $\mathrm{O}$ & 1.352377 & -8.350409 & 1.246258 \\
\hline $\mathrm{Zn}$ & -0.568848 & -8.437593 & 1.623651 \\
\hline $\mathrm{O}$ & -1.206700 & -9.638201 & 3.038976 \\
\hline $\mathrm{Zn}$ & 0.151624 & -10.863646 & 3.667967 \\
\hline $\mathrm{Zn}$ & 2.572297 & -11.359802 & 4.752223 \\
\hline $\mathrm{Zn}$ & 5.189803 & -9.445925 & 4.368286 \\
\hline $\mathrm{O}$ & 6.721051 & -8.360461 & 4.930083 \\
\hline $\mathrm{Zn}$ & 6.278107 & -7.409939 & 6.595323 \\
\hline $\mathrm{O}$ & 5.217558 & -8.381235 & 7.938838 \\
\hline $\mathrm{Zn}$ & 4.751721 & -7.323317 & 9.496489 \\
\hline $\mathrm{O}$ & 2.950172 & -7.279620 & 10.405865 \\
\hline $\mathrm{Zn}$ & 1.607933 & -7.802549 & 9.099489 \\
\hline $\mathrm{O}$ & 0.031230 & -6.666246 & 8.827541 \\
\hline $\mathrm{Zn}$ & 0.104244 & -4.769917 & 9.275230 \\
\hline $\mathrm{O}$ & 1.816036 & -3.962488 & 9.825357 \\
\hline $\mathrm{Zn}$ & 3.148170 & -5.287350 & 10.300118 \\
\hline $\mathrm{O}$ & 5.082093 & -5.362112 & 9.811000 \\
\hline $\mathrm{Zn}$ & 5.276219 & -4.593021 & 8.026352 \\
\hline $\mathrm{Zn}$ & 4.848072 & -1.978302 & 6.131973 \\
\hline $\mathrm{O}$ & 4.043993 & -0.180685 & 6.063600 \\
\hline $\mathrm{Zn}$ & 2.089907 & -0.270176 & 6.432600 \\
\hline $\mathrm{O}$ & 1.248656 & -1.296355 & 7.840172 \\
\hline $\mathrm{Zn}$ & -0.444861 & -2.216893 & 7.479238 \\
\hline $\mathrm{O}$ & -1.300534 & -3.565548 & 8.614580 \\
\hline $\mathrm{Zn}$ & -2.801055 & -4.483955 & 7.805673 \\
\hline $\mathrm{Zn}$ & -1.419489 & -7.358689 & 7.709103 \\
\hline $\mathrm{Zn}$ & 0.452339 & -9.797622 & 6.646215 \\
\hline $\mathrm{O}$ & 1.972582 & -9.002895 & 7.597954 \\
\hline
\end{tabular}

$-7.179637$

$-6.969870$

$-6.971820$

\section{(ZnO)}




\begin{tabular}{|c|c|c|c|}
\hline $\mathrm{Zn}$ & 3.809758 & -9.497352 & 7.125300 \\
\hline $\mathrm{O}$ & 4.164492 & -10.618868 & 5.558719 \\
\hline $\mathrm{Zn}$ & 3.603911 & -0.212114 & 4.142498 \\
\hline $\mathrm{O}$ & 1.559497 & -0.066451 & 4.458670 \\
\hline $\mathrm{Zn}$ & 0.656084 & -1.859777 & 4.441502 \\
\hline $\mathrm{Zn}$ & -0.365075 & -3.563154 & 2.174026 \\
\hline $\mathrm{O}$ & -0.563212 & -3.720230 & 0.234802 \\
\hline $\mathrm{Zn}$ & -0.707226 & -5.710754 & -0.099006 \\
\hline $\mathrm{O}$ & 1.094871 & -5.809073 & -0.973298 \\
\hline $\mathrm{O}$ & 1.342394 & -3.013115 & 3.008213 \\
\hline $\mathrm{Zn}$ & 2.484694 & -2.684287 & 8.494399 \\
\hline $\mathrm{O}$ & 4.007335 & -3.240241 & 7.388791 \\
\hline $\mathrm{O}$ & 6.409869 & -5.459576 & 6.675242 \\
\hline $\mathrm{Zn}$ & 6.957702 & -4.380797 & 5.131352 \\
\hline $\mathrm{O}$ & 6.056036 & -2.680331 & 4.764856 \\
\hline $\mathrm{O}$ & 7.967755 & -5.227212 & 3.685115 \\
\hline $\mathrm{Zn}$ & 7.107613 & -5.064301 & 1.863225 \\
\hline $\mathrm{Zn}$ & 5.046172 & -7.426673 & 1.629827 \\
\hline $\mathrm{O}$ & 3.821764 & -6.031742 & 0.982802 \\
\hline $\mathrm{Zn}$ & 7.384119 & -7.128419 & 3.594823 \\
\hline $\mathrm{O}$ & 6.961110 & -7.042047 & 1.635954 \\
\hline $\mathrm{O}$ & 6.010992 & -3.562284 & 1.321667 \\
\hline $\mathrm{O}$ & -0.887519 & -2.177466 & 5.571024 \\
\hline $\mathrm{Zn}$ & -2.276214 & -3.384673 & 4.897695 \\
\hline $\mathrm{O}$ & -3.681365 & -4.098977 & 6.046929 \\
\hline $\mathrm{Zn}$ & -3.574768 & -6.085577 & 5.752406 \\
\hline $\mathrm{O}$ & -3.155438 & -6.454581 & 7.683883 \\
\hline $\mathrm{O}$ & -1.783444 & -4.348619 & 3.269812 \\
\hline $\mathrm{Zn}$ & -2.369709 & -6.141759 & 2.742533 \\
\hline $\mathrm{O}$ & -3.217907 & -7.168975 & 4.190602 \\
\hline $\mathrm{Zn}$ & -2.012441 & -8.681644 & 4.552401 \\
\hline $\mathrm{O}$ & -1.127387 & -8.697769 & 6.307798 \\
\hline $\mathrm{O}$ & -1.679197 & -6.910359 & 1.074814 \\
\hline $\mathrm{O}$ & 1.929435 & -11.313715 & 2.853469 \\
\hline $\mathrm{Zn}$ & 2.581297 & -9.629028 & 2.096223 \\
\hline $\mathrm{O}$ & 4.282484 & -8.869363 & 2.718041 \\
\hline $\mathrm{O}$ & 0.718428 & -11.364275 & 5.519383 \\
\hline $\mathrm{H}$ & 0.368347 & -0.870358 & -0.292882 \\
\hline $\mathrm{H}$ & -1.232118 & -0.116480 & -0.181233 \\
\hline $\mathrm{H}$ & 0.221794 & 0.897171 & -0.276176 \\
\hline $\mathrm{H}$ & -0.612501 & 0.819946 & 2.067504 \\
\hline $\mathrm{H}$ & -0.543779 & -0.950837 & 2.046334 \\
\hline $\mathrm{H}$ & 1.371096 & 0.056017 & 3.125817 \\
\hline
\end{tabular}


$(\mathrm{ZnO})_{36}-\mathrm{CH}_{3}\left(\mathrm{CH}_{2}\right)_{2} \mathrm{OH}$

\begin{tabular}{|c|c|c|c|}
\hline $\mathrm{C}$ & -1.595251 & -0.137097 & 0.082957 \\
\hline $\mathrm{C}$ & -0.150254 & -0.173030 & 0.574057 \\
\hline $\mathrm{C}$ & -0.055614 & -0.514132 & 2.051074 \\
\hline $\mathrm{O}$ & 1.308485 & -0.537521 & 2.451016 \\
\hline $\mathrm{Zn}$ & 2.515833 & -2.124426 & 1.596514 \\
\hline $\mathrm{Zn}$ & 3.729947 & -0.978528 & 4.252941 \\
\hline $\mathrm{O}$ & 1.746891 & -1.318385 & 4.741464 \\
\hline $\mathrm{Zn}$ & 2.529418 & -1.932756 & 6.536185 \\
\hline $\mathrm{O}$ & 4.388360 & -1.429458 & 6.054146 \\
\hline $\mathrm{Zn}$ & 5.382250 & -3.076181 & 5.672185 \\
\hline $\mathrm{Zn}$ & 6.177217 & -6.063791 & 6.686729 \\
\hline $\mathrm{O}$ & 6.177399 & -7.327423 & 8.176466 \\
\hline $\mathrm{Zn}$ & 5.989078 & -9.178983 & 7.410911 \\
\hline $\mathrm{Zn}$ & 2.848496 & -9.912737 & 7.264685 \\
\hline $\mathrm{O}$ & 3.264834 & -10.887829 & 5.616094 \\
\hline $\mathrm{Zn}$ & 1.718810 & -11.224100 & 4.458256 \\
\hline $\mathrm{O}$ & 1.928943 & -12.389444 & 2.904835 \\
\hline $\mathrm{Zn}$ & 1.166628 & -11.451460 & 1.294355 \\
\hline $\mathrm{O}$ & 2.931589 & -11.320589 & 0.372567 \\
\hline $\mathrm{Zn}$ & 3.308993 & -9.411167 & 0.129889 \\
\hline $\mathrm{Zn}$ & 3.714690 & -11.911156 & 2.122125 \\
\hline $\mathrm{O}$ & 5.338230 & -11.238023 & 2.925500 \\
\hline $\mathrm{Zn}$ & 5.067069 & -10.849952 & 4.839970 \\
\hline $\mathrm{O}$ & 6.478625 & -9.714320 & 5.616858 \\
\hline $\mathrm{Zn}$ & 7.299454 & -8.324067 & 4.490512 \\
\hline $\mathrm{O}$ & 7.229439 & -6.467814 & 5.083934 \\
\hline $\mathrm{Zn}$ & 7.565379 & -4.971840 & 3.854942 \\
\hline $\mathrm{Zn}$ & 5.816880 & -2.620658 & 2.392205 \\
\hline $\mathrm{O}$ & 6.183254 & -3.295805 & 0.588840 \\
\hline $\mathrm{Zn}$ & 7.453829 & -4.751679 & 0.656970 \\
\hline $\mathrm{O}$ & 8.561187 & -5.334728 & 2.218326 \\
\hline $\mathrm{Zn}$ & 8.171213 & -7.210508 & 1.605931 \\
\hline $\mathrm{O}$ & 7.404373 & -6.586061 & -0.140891 \\
\hline $\mathrm{Zn}$ & 5.509263 & -7.027616 & -0.081055 \\
\hline $\mathrm{O}$ & 4.985670 & -8.688654 & 0.835049 \\
\hline $\mathrm{Zn}$ & 6.170323 & -9.712659 & 2.023028 \\
\hline $\mathrm{O}$ & 7.802538 & -8.804217 & 2.649481 \\
\hline $\mathrm{O}$ & 1.528579 & -3.659294 & 2.441919 \\
\hline $\mathrm{Zn}$ & -0.243689 & -4.070784 & 1.690161 \\
\hline $\mathrm{O}$ & -1.525936 & -5.116612 & 2.721183 \\
\hline $\mathrm{Zn}$ & -1.749089 & -4.816623 & 4.651912 \\
\hline $\mathrm{O}$ & -2.931085 & -5.963227 & 5.686284 \\
\hline $\mathrm{Zn}$ & -2.763733 & -7.772413 & 4.825755 \\
\hline
\end{tabular}




\begin{tabular}{|c|c|c|c|}
\hline $\mathrm{Zn}$ & -0.856672 & -9.610677 & 2.978168 \\
\hline $\mathrm{O}$ & -0.269471 & -10.164077 & 1.188899 \\
\hline $\mathrm{Zn}$ & 0.076423 & -8.614033 & 0.043569 \\
\hline $\mathrm{O}$ & -1.239573 & -7.146736 & -0.028918 \\
\hline $\mathrm{Zn}$ & -0.466304 & -5.630376 & -0.970449 \\
\hline $\mathrm{O}$ & -0.490362 & -3.759301 & -0.222051 \\
\hline $\mathrm{Zn}$ & 1.296930 & -3.500084 & -1.109821 \\
\hline $\mathrm{Zn}$ & 4.386511 & -3.709961 & -0.104658 \\
\hline $\mathrm{O}$ & 4.109096 & -5.663190 & -0.230483 \\
\hline $\mathrm{Zn}$ & 2.469256 & -6.376907 & -1.010428 \\
\hline $\mathrm{O}$ & 1.917473 & -8.179257 & -0.476819 \\
\hline $\mathrm{Zn}$ & 0.992758 & -3.101919 & 4.230749 \\
\hline $\mathrm{O}$ & -0.296937 & -3.805955 & 5.501080 \\
\hline $\mathrm{Zn}$ & 0.280727 & -4.261034 & 7.321640 \\
\hline $\mathrm{Zn}$ & 3.331184 & -4.667648 & 7.931632 \\
\hline $\mathrm{O}$ & 4.783671 & -4.688652 & 6.624444 \\
\hline $\mathrm{O}$ & 2.816281 & -2.556875 & -0.342805 \\
\hline $\mathrm{O}$ & 4.210953 & -1.509113 & 2.464891 \\
\hline $\mathrm{O}$ & -0.333468 & -5.955605 & 8.093068 \\
\hline $\mathrm{Zn}$ & 1.216642 & -7.121037 & 8.382639 \\
\hline $\mathrm{Zn}$ & 4.324228 & -7.627304 & 8.891948 \\
\hline $\mathrm{O}$ & 4.349658 & -9.579033 & 8.477035 \\
\hline $\mathrm{Zn}$ & -1.842094 & -6.746786 & 7.176236 \\
\hline $\mathrm{Zn}$ & -0.263603 & -9.318562 & 6.329625 \\
\hline $\mathrm{O}$ & 1.205840 & -8.909203 & 7.563699 \\
\hline $\mathrm{O}$ & 1.928215 & -3.282522 & 7.778459 \\
\hline $\mathrm{O}$ & -2.076331 & -8.620780 & 6.518153 \\
\hline $\mathrm{O}$ & -2.288876 & -8.280593 & 3.017876 \\
\hline $\mathrm{Zn}$ & -1.910012 & -6.791962 & 1.781832 \\
\hline $\mathrm{O}$ & 1.190901 & -5.292872 & -2.015048 \\
\hline $\mathrm{O}$ & 0.284535 & -9.891183 & 4.528854 \\
\hline $\mathrm{O}$ & 2.917827 & -6.299743 & 8.944543 \\
\hline $\mathrm{O}$ & 6.472778 & -3.367386 & 4.082063 \\
\hline $\mathrm{H}$ & 0.421170 & -0.918779 & 0.010665 \\
\hline $\mathrm{H}$ & 0.331568 & 0.795472 & 0.408973 \\
\hline $\mathrm{H}$ & -0.602877 & 0.227139 & 2.645887 \\
\hline $\mathrm{H}$ & -0.522318 & -1.495752 & 2.230771 \\
\hline $\mathrm{H}$ & 1.424104 & -0.824748 & 3.503481 \\
\hline $\mathrm{H}$ & -1.639549 & 0.090343 & -0.984198 \\
\hline $\mathrm{H}$ & -2.087026 & -1.101006 & 0.238769 \\
\hline $\mathrm{H}$ & -2.170090 & 0.627471 & 0.612694 \\
\hline
\end{tabular}

$(\mathrm{ZnO})_{36}-\mathrm{CH}_{3}\left(\mathrm{CH}_{2}\right)_{3} \mathrm{OH}$ 


\begin{tabular}{|c|c|c|c|}
\hline $\mathrm{C}$ & -0.620362 & -0.256996 & 0.460813 \\
\hline $\mathrm{C}$ & -0.305092 & -0.267264 & 1.954939 \\
\hline $\mathrm{C}$ & 1.185131 & -0.072261 & 2.234946 \\
\hline $\mathrm{C}$ & 1.508568 & -0.211672 & 3.711312 \\
\hline $\mathrm{O}$ & 2.910666 & -0.110147 & 3.932375 \\
\hline $\mathrm{Zn}$ & 4.127959 & -1.868934 & 3.348386 \\
\hline $\mathrm{O}$ & 5.800703 & -1.174102 & 4.181112 \\
\hline $\mathrm{Zn}$ & 7.354405 & -2.347062 & 4.390740 \\
\hline $\mathrm{O}$ & 7.880634 & -2.872406 & 6.204605 \\
\hline $\mathrm{Zn}$ & 8.741967 & -4.647224 & 6.266444 \\
\hline $\mathrm{Zn}$ & 9.484356 & -7.126271 & 4.288518 \\
\hline $\mathrm{O}$ & 8.860008 & -8.482485 & 5.511511 \\
\hline $\mathrm{Zn}$ & 7.406543 & -9.610954 & 4.806028 \\
\hline $\mathrm{O}$ & 6.491553 & -8.812715 & 3.263767 \\
\hline $\mathrm{Zn}$ & 7.060786 & -7.134853 & 2.415806 \\
\hline $\mathrm{Zn}$ & 4.067384 & -6.491866 & 1.402310 \\
\hline $\mathrm{O}$ & 2.923195 & -5.481820 & 0.187294 \\
\hline $\mathrm{Zn}$ & 3.056368 & -3.587397 & 0.800342 \\
\hline $\mathrm{O}$ & 4.553085 & -2.604410 & 1.544085 \\
\hline $\mathrm{Zn}$ & 6.085879 & -3.777667 & 1.902760 \\
\hline $\mathrm{O}$ & 5.732311 & -5.723453 & 2.089302 \\
\hline $\mathrm{Zn}$ & 5.357493 & -0.463290 & 5.910475 \\
\hline $\mathrm{Zn}$ & 6.800245 & -2.357386 & 7.752403 \\
\hline $\mathrm{O}$ & 6.139362 & -3.711079 & 8.996099 \\
\hline $\mathrm{Zn}$ & 4.582022 & -3.429619 & 10.144555 \\
\hline $\mathrm{O}$ & 4.022582 & -4.965658 & 11.236663 \\
\hline $\mathrm{Zn}$ & 5.393999 & -6.329974 & 11.366863 \\
\hline $\mathrm{O}$ & 5.239952 & -8.310074 & 11.134599 \\
\hline $\mathrm{Zn}$ & 3.871337 & -8.811746 & 9.844535 \\
\hline $\mathrm{O}$ & 2.349272 & -7.564482 & 9.841474 \\
\hline $\mathrm{Zn}$ & 0.828527 & -8.173325 & 8.778562 \\
\hline $\mathrm{O}$ & 1.204873 & -9.234911 & 7.173151 \\
\hline $\mathrm{Zn}$ & 0.223225 & -8.981491 & 5.509286 \\
\hline $\mathrm{Zn}$ & -0.501248 & -6.278913 & 3.929106 \\
\hline $\mathrm{O}$ & -1.094767 & -7.525994 & 5.315890 \\
\hline $\mathrm{Zn}$ & -1.514181 & -6.815860 & 7.066685 \\
\hline $\mathrm{O}$ & -0.946373 & -7.369063 & 8.888065 \\
\hline $\mathrm{Zn}$ & -0.680187 & -5.405314 & 9.254173 \\
\hline $\mathrm{O}$ & 0.811963 & -4.586775 & 10.186929 \\
\hline $\mathrm{Zn}$ & 2.329315 & -5.764865 & 10.622335 \\
\hline $\mathrm{Zn}$ & 1.628679 & -3.115151 & 9.174707 \\
\hline $\mathrm{O}$ & 3.259644 & -2.102260 & 9.566838 \\
\hline $\mathrm{Zn}$ & 3.960559 & -0.894166 & 8.225965 \\
\hline $\mathrm{Zn}$ & 2.426909 & -2.364562 & 5.993847 \\
\hline $\mathrm{O}$ & 1.148289 & -3.045000 & 7.277216 \\
\hline
\end{tabular}




\begin{tabular}{|c|c|c|c|}
\hline $\mathrm{Zn}$ & -0.316877 & -4.031689 & 6.420248 \\
\hline $\mathrm{O}$ & -1.636652 & -4.877464 & 7.569795 \\
\hline $\mathrm{O}$ & 3.016554 & -3.228625 & 4.331675 \\
\hline $\mathrm{Zn}$ & 1.318932 & -3.647091 & 3.426770 \\
\hline $\mathrm{O}$ & 1.170707 & -3.563333 & 1.484957 \\
\hline $\mathrm{Zn}$ & 1.120724 & -5.524041 & 1.082406 \\
\hline $\mathrm{O}$ & 0.290014 & -6.864086 & 2.218112 \\
\hline $\mathrm{Zn}$ & 1.444770 & -8.411648 & 2.541706 \\
\hline $\mathrm{O}$ & 3.356055 & -8.150806 & 2.151929 \\
\hline $\mathrm{Zn}$ & 4.699780 & -9.408761 & 2.775356 \\
\hline $\mathrm{Zn}$ & 4.800149 & -11.623522 & 5.149579 \\
\hline $\mathrm{Zn}$ & 6.098480 & -10.172002 & 7.741432 \\
\hline $\mathrm{Zn}$ & 8.354927 & -7.902814 & 7.307937 \\
\hline $\mathrm{O}$ & 8.103229 & -5.961486 & 7.567098 \\
\hline $\mathrm{Zn}$ & 7.331444 & -5.249215 & 9.214148 \\
\hline $\mathrm{Zn}$ & 7.038742 & -8.187042 & 10.249287 \\
\hline $\mathrm{O}$ & 7.509643 & -9.107396 & 8.606597 \\
\hline $\mathrm{O}$ & 3.336542 & -0.614812 & 6.298172 \\
\hline $\mathrm{O}$ & 5.894591 & -0.604188 & 7.794546 \\
\hline $\mathrm{O}$ & 7.325814 & -6.301660 & 10.859112 \\
\hline $\mathrm{O}$ & 4.243516 & -9.984828 & 8.332815 \\
\hline $\mathrm{Zn}$ & 2.699611 & -10.491548 & 7.239696 \\
\hline $\mathrm{Zn}$ & 2.332033 & -11.091320 & 4.132552 \\
\hline $\mathrm{O}$ & 0.933510 & -9.772940 & 3.857958 \\
\hline $\mathrm{O}$ & 2.951253 & -11.864817 & 5.879413 \\
\hline $\mathrm{O}$ & 4.150127 & -11.204512 & 3.298139 \\
\hline $\mathrm{O}$ & 6.407994 & -10.874709 & 5.924912 \\
\hline $\mathrm{Zn}$ & 8.962542 & -4.774791 & 3.018555 \\
\hline $\mathrm{O}$ & 8.954587 & -6.672160 & 2.410378 \\
\hline $\mathrm{O}$ & 9.851536 & -5.211468 & 4.765313 \\
\hline $\mathrm{O}$ & 7.817353 & -3.254992 & 2.705036 \\
\hline $\mathrm{O}$ & -0.078688 & -4.456865 & 4.525039 \\
\hline $\mathrm{H}$ & 1.764358 & -0.817540 & 1.677463 \\
\hline $\mathrm{H}$ & 1.507251 & 0.915336 & 1.887964 \\
\hline $\mathrm{H}$ & 0.988949 & 0.564537 & 4.286366 \\
\hline $\mathrm{H}$ & 1.144557 & -1.187296 & 4.071804 \\
\hline $\mathrm{H}$ & 3.085757 & -0.239772 & 5.000271 \\
\hline $\mathrm{H}$ & -0.630913 & -1.220519 & 2.385297 \\
\hline $\mathrm{H}$ & -0.875943 & 0.519400 & 2.459525 \\
\hline $\mathrm{H}$ & -1.690176 & -0.385098 & 0.279407 \\
\hline $\mathrm{H}$ & -0.310172 & 0.686068 & 0.002503 \\
\hline $\mathrm{H}$ & -0.093331 & -1.069327 & -0.046894 \\
\hline
\end{tabular}

$(\mathrm{ZnO})_{36}-\mathrm{CH}_{3}\left(\mathrm{CH}_{2}\right)_{4} \mathrm{OH}$ 


\begin{tabular}{|c|c|c|c|}
\hline $\mathrm{C}$ & 0.072150 & 0.529505 & 0.155159 \\
\hline $\mathrm{C}$ & 0.078121 & 0.445535 & 1.679974 \\
\hline $\mathrm{C}$ & 1.457833 & 0.093538 & 2.233083 \\
\hline $\mathrm{C}$ & 1.482359 & 0.013787 & 3.758497 \\
\hline $\mathrm{C}$ & 2.870035 & -0.291215 & 4.290065 \\
\hline $\mathrm{O}$ & 2.874906 & -0.290472 & 5.713048 \\
\hline $\mathrm{Zn}$ & 2.728437 & 1.663370 & 6.669561 \\
\hline $\mathrm{O}$ & 4.299590 & 2.496287 & 5.713184 \\
\hline $\mathrm{Zn}$ & 5.604057 & 1.063877 & 5.395202 \\
\hline $\mathrm{Zn}$ & 8.771030 & 1.056337 & 4.909234 \\
\hline $\mathrm{O}$ & 8.694516 & 0.238574 & 6.706095 \\
\hline $\mathrm{Zn}$ & 9.385794 & 1.512464 & 8.032315 \\
\hline $\mathrm{O}$ & 10.967215 & 2.597203 & 7.618807 \\
\hline $\mathrm{Zn}$ & 11.413315 & 3.751424 & 9.104643 \\
\hline $\mathrm{O}$ & 11.712816 & 5.731094 & 8.919472 \\
\hline $\mathrm{Zn}$ & 10.551351 & 5.880619 & 10.554897 \\
\hline $\mathrm{O}$ & 10.602010 & 3.917725 & 10.921287 \\
\hline $\mathrm{Zn}$ & 8.697366 & 3.503596 & 10.778465 \\
\hline $\mathrm{O}$ & 8.173621 & 2.195938 & 9.416736 \\
\hline $\mathrm{Zn}$ & 6.564901 & 1.216966 & 9.962722 \\
\hline $\mathrm{Zn}$ & 4.534010 & -0.358812 & 8.413584 \\
\hline $\mathrm{Zn}$ & 3.325018 & 2.179105 & 9.996356 \\
\hline $\mathrm{Zn}$ & 1.530502 & 4.136844 & 8.233013 \\
\hline $\mathrm{O}$ & 1.221750 & 2.969665 & 6.685451 \\
\hline $\mathrm{Zn}$ & 0.995655 & 3.991645 & 5.060645 \\
\hline $\mathrm{Zn}$ & 2.047553 & 6.778256 & 6.012788 \\
\hline $\mathrm{O}$ & 3.392688 & 8.087203 & 5.472905 \\
\hline $\mathrm{Zn}$ & 4.241864 & 9.132868 & 6.895987 \\
\hline $\mathrm{O}$ & 4.267583 & 8.386331 & 8.704224 \\
\hline $\mathrm{Zn}$ & 5.818675 & 8.764045 & 9.833254 \\
\hline $\mathrm{O}$ & 7.395943 & 9.633586 & 9.066516 \\
\hline $\mathrm{Zn}$ & 7.089397 & 10.563770 & 7.400150 \\
\hline $\mathrm{O}$ & 5.339025 & 10.668434 & 6.428766 \\
\hline $\mathrm{Zn}$ & 6.392052 & 10.223343 & 4.787102 \\
\hline $\mathrm{Zn}$ & 7.224986 & 7.755979 & 2.823369 \\
\hline $\mathrm{O}$ & 6.964751 & 6.306440 & 1.510046 \\
\hline $\mathrm{Zn}$ & 5.279270 & 5.441803 & 2.050386 \\
\hline $\mathrm{Zn}$ & 8.392856 & 4.999704 & 1.475603 \\
\hline $\mathrm{O}$ & 10.193760 & 5.110453 & 2.351406 \\
\hline $\mathrm{Zn}$ & 10.056832 & 6.076031 & 4.041951 \\
\hline $\mathrm{O}$ & 8.675552 & 7.457164 & 4.127906 \\
\hline $\mathrm{Zn}$ & 8.946923 & 8.592249 & 5.691688 \\
\hline $\mathrm{O}$ & 9.512788 & 7.720563 & 7.352255 \\
\hline $\mathrm{Zn}$ & 10.891511 & 6.330138 & 7.255727 \\
\hline
\end{tabular}




\begin{tabular}{|c|c|c|c|}
\hline $\mathrm{O}$ & 10.960237 & 5.297648 & 5.591661 \\
\hline $\mathrm{Zn}$ & 10.956591 & 3.350553 & 5.804187 \\
\hline $\mathrm{O}$ & 10.278127 & 2.177417 & 4.370390 \\
\hline $\mathrm{Zn}$ & 9.972240 & 3.166639 & 2.737361 \\
\hline $\mathrm{O}$ & 8.363985 & 2.999796 & 1.561995 \\
\hline $\mathrm{Zn}$ & 6.844143 & 2.434484 & 2.630681 \\
\hline $\mathrm{O}$ & 6.999136 & 1.084385 & 4.054600 \\
\hline $\mathrm{Zn}$ & 3.803474 & 3.053620 & 3.898456 \\
\hline $\mathrm{O}$ & 2.004015 & 3.568394 & 3.375362 \\
\hline $\mathrm{Zn}$ & 2.159370 & 5.556625 & 3.125119 \\
\hline $\mathrm{O}$ & 0.915536 & 5.943910 & 4.648627 \\
\hline $\mathrm{O}$ & 3.138827 & 0.960409 & 8.483675 \\
\hline $\mathrm{O}$ & 5.305757 & 3.625224 & 2.777346 \\
\hline $\mathrm{O}$ & 8.158092 & 10.378731 & 5.715225 \\
\hline $\mathrm{O}$ & 5.855404 & 9.073799 & 3.323075 \\
\hline $\mathrm{Zn}$ & 4.204747 & 8.078012 & 3.683887 \\
\hline $\mathrm{O}$ & 3.735003 & 6.537360 & 2.566726 \\
\hline $\mathrm{O}$ & 2.055470 & 5.991868 & 7.806952 \\
\hline $\mathrm{Zn}$ & 2.751688 & 7.231952 & 9.154746 \\
\hline $\mathrm{O}$ & 2.510520 & 6.896125 & 11.074604 \\
\hline $\mathrm{Zn}$ & 4.322022 & 6.861278 & 11.910483 \\
\hline $\mathrm{Zn}$ & 7.474238 & 6.574207 & 11.498513 \\
\hline $\mathrm{O}$ & 7.332726 & 4.623405 & 11.623616 \\
\hline $\mathrm{Zn}$ & 5.569125 & 3.792459 & 11.833801 \\
\hline $\mathrm{O}$ & 5.106494 & 2.188338 & 10.817846 \\
\hline $\mathrm{Zn}$ & 2.528536 & 4.914183 & 11.327157 \\
\hline $\mathrm{O}$ & 1.958131 & 3.575802 & 10.064760 \\
\hline $\mathrm{O}$ & 4.151896 & 4.957871 & 12.498140 \\
\hline $\mathrm{O}$ & 5.960675 & 7.820280 & 11.549641 \\
\hline $\mathrm{Zn}$ & 8.847158 & 8.313358 & 9.096014 \\
\hline $\mathrm{O}$ & 9.156709 & 7.213689 & 10.681356 \\
\hline $\mathrm{Zn}$ & 7.048354 & -0.574973 & 7.319374 \\
\hline $\mathrm{O}$ & 5.197666 & -0.578103 & 6.459938 \\
\hline $\mathrm{O}$ & 6.344598 & -0.566808 & 9.180667 \\
\hline $\mathrm{H}$ & 1.144184 & 0.966674 & 4.181796 \\
\hline $\mathrm{H}$ & 0.790969 & -0.761127 & 4.106345 \\
\hline $\mathrm{H}$ & 3.208103 & -1.268040 & 3.923368 \\
\hline $\mathrm{H}$ & 3.583124 & 0.463329 & 3.917750 \\
\hline $\mathrm{H}$ & 3.899237 & -0.506797 & 6.036601 \\
\hline $\mathrm{H}$ & 2.177761 & 0.848850 & 1.895914 \\
\hline $\mathrm{H}$ & 1.782807 & -0.865340 & 1.811471 \\
\hline $\mathrm{H}$ & -0.647105 & -0.305250 & 2.011725 \\
\hline $\mathrm{H}$ & -0.240480 & 1.404390 & 2.102319 \\
\hline $\mathrm{H}$ & -0.919269 & 0.781123 & -0.228838 \\
\hline $\mathrm{H}$ & 0.770851 & 1.295609 & -0.192502 \\
\hline
\end{tabular}


$(\mathrm{ZnO})_{36}-\mathrm{CH}_{3}\left(\mathrm{CH}_{2}\right)_{7} \mathrm{OH}$

\begin{tabular}{|c|c|c|c|}
\hline $\mathrm{C}$ & -0.701303 & 1.227668 & 0.425902 \\
\hline $\mathrm{C}$ & -0.600380 & 0.668852 & 1.843981 \\
\hline $\mathrm{C}$ & 0.844743 & 0.588647 & 2.332942 \\
\hline $\mathrm{C}$ & 0.978536 & 0.035508 & 3.751265 \\
\hline $\mathrm{C}$ & 2.433094 & -0.040645 & 4.210919 \\
\hline $\mathrm{C}$ & 2.600674 & -0.583583 & 5.629060 \\
\hline $\mathrm{C}$ & 4.068494 & -0.615856 & 6.048279 \\
\hline $\mathrm{C}$ & 4.288637 & -1.188194 & 7.435052 \\
\hline $\mathrm{O}$ & 5.685068 & -1.216829 & 7.707633 \\
\hline $\mathrm{Zn}$ & 6.866739 & -2.732751 & 6.509586 \\
\hline $\mathrm{O}$ & 8.535165 & -2.202567 & 7.457779 \\
\hline $\mathrm{Zn}$ & 10.079123 & -3.338064 & 7.126234 \\
\hline $\mathrm{Zn}$ & 8.840473 & -4.086055 & 4.270687 \\
\hline $\mathrm{O}$ & 7.248900 & -2.987774 & 4.562137 \\
\hline $\mathrm{Zn}$ & 5.718504 & -3.542418 & 3.524871 \\
\hline $\mathrm{Zn}$ & 6.933450 & -6.394717 & 2.843800 \\
\hline $\mathrm{O}$ & 6.375558 & -8.202960 & 3.334864 \\
\hline $\mathrm{Zn}$ & 4.552636 & -8.686000 & 3.825845 \\
\hline $\mathrm{Zn}$ & 2.432055 & -7.252949 & 5.851901 \\
\hline $\mathrm{O}$ & 2.082956 & -8.931678 & 6.792626 \\
\hline $\mathrm{Zn}$ & 3.355859 & -10.378496 & 6.383290 \\
\hline $\mathrm{O}$ & 4.424027 & -11.181919 & 7.816940 \\
\hline $\mathrm{Zn}$ & 3.990595 & -10.649792 & 9.643576 \\
\hline $\mathrm{O}$ & 2.276524 & -9.894419 & 10.153502 \\
\hline $\mathrm{Zn}$ & 2.469998 & -8.134221 & 11.086276 \\
\hline $\mathrm{Zn}$ & 5.486941 & -8.858555 & 12.121465 \\
\hline $\mathrm{O}$ & 7.210403 & -8.242490 & 12.834771 \\
\hline $\mathrm{Zn}$ & 8.590305 & -9.594363 & 12.725671 \\
\hline $\mathrm{O}$ & 8.517718 & -11.392551 & 11.854990 \\
\hline $\mathrm{Zn}$ & 7.130277 & -11.344516 & 10.487684 \\
\hline $\mathrm{O}$ & 5.510840 & -10.318410 & 10.829011 \\
\hline $\mathrm{Zn}$ & 8.131853 & -2.122671 & 9.324793 \\
\hline $\mathrm{Zn}$ & 9.828811 & -4.454366 & 10.434467 \\
\hline $\mathrm{O}$ & 9.430856 & -6.090443 & 11.422047 \\
\hline $\mathrm{Zn}$ & 7.724704 & -6.424432 & 12.326956 \\
\hline $\mathrm{O}$ & 6.310304 & -5.055234 & 12.198153 \\
\hline $\mathrm{Zn}$ & 4.571726 & -5.782167 & 11.633382 \\
\hline $\mathrm{O}$ & 4.000729 & -7.580772 & 12.137523 \\
\hline $\mathrm{O}$ & 5.719735 & -4.272847 & 7.106966 \\
\hline $\mathrm{Zn}$ & 5.186307 & -3.963281 & 8.958501 \\
\hline
\end{tabular}




\begin{tabular}{|c|c|c|c|}
\hline $\mathrm{O}$ & 3.898918 & -5.000593 & 9.966329 \\
\hline $\mathrm{Zn}$ & 2.491226 & -5.796862 & 8.860178 \\
\hline $\mathrm{Zn}$ & 1.522619 & -8.825831 & 8.635217 \\
\hline $\mathrm{O}$ & 1.397433 & -7.157990 & 9.720098 \\
\hline $\mathrm{O}$ & 6.161656 & -2.463337 & 9.809911 \\
\hline $\mathrm{Zn}$ & 6.944205 & -3.392157 & 11.446051 \\
\hline $\mathrm{O}$ & 8.805438 & -2.878796 & 11.002623 \\
\hline $\mathrm{Zn}$ & 11.779814 & -5.995896 & 8.311254 \\
\hline $\mathrm{Zn}$ & 12.482470 & -7.699776 & 5.682288 \\
\hline $\mathrm{Zn}$ & 10.418877 & -10.173068 & 5.329878 \\
\hline $\mathrm{O}$ & 9.563371 & -9.069977 & 3.960927 \\
\hline $\mathrm{Zn}$ & 10.101838 & -7.239988 & 3.514443 \\
\hline $\mathrm{O}$ & 11.926920 & -6.751519 & 4.007919 \\
\hline $\mathrm{Zn}$ & 11.870091 & -5.123961 & 5.164443 \\
\hline $\mathrm{O}$ & 10.502331 & -3.772193 & 5.264193 \\
\hline $\mathrm{O}$ & 10.639319 & -4.445577 & 8.650738 \\
\hline $\mathrm{Zn}$ & 10.212651 & -10.861048 & 10.945894 \\
\hline $\mathrm{Zn}$ & 11.559028 & -9.366623 & 8.385743 \\
\hline $\mathrm{O}$ & 11.808282 & -9.345132 & 6.434346 \\
\hline $\mathrm{Zn}$ & 10.620781 & -7.605552 & 11.068028 \\
\hline $\mathrm{O}$ & 11.481788 & -7.640893 & 9.302286 \\
\hline $\mathrm{O}$ & 10.451962 & -9.243272 & 12.091240 \\
\hline $\mathrm{Zn}$ & 9.438594 & -11.810208 & 7.933319 \\
\hline $\mathrm{O}$ & 10.793737 & -11.012636 & 9.112074 \\
\hline $\mathrm{O}$ & 7.621517 & -11.852739 & 8.667479 \\
\hline $\mathrm{Zn}$ & 6.084740 & -12.156859 & 7.503447 \\
\hline $\mathrm{Zn}$ & 8.074140 & -12.385502 & 4.971513 \\
\hline $\mathrm{O}$ & 9.632516 & -11.850122 & 5.984398 \\
\hline $\mathrm{O}$ & 6.305213 & -12.938313 & 5.729499 \\
\hline $\mathrm{Zn}$ & 5.508732 & -11.752231 & 4.340630 \\
\hline $\mathrm{O}$ & 4.108132 & -10.434689 & 4.572627 \\
\hline $\mathrm{O}$ & 7.256146 & -11.450692 & 3.415287 \\
\hline $\mathrm{Zn}$ & 7.758016 & -9.578677 & 3.417846 \\
\hline $\mathrm{O}$ & 12.833928 & -6.005602 & 6.675037 \\
\hline $\mathrm{Zn}$ & 4.013842 & -4.448403 & 6.137793 \\
\hline $\mathrm{O}$ & 3.892952 & -3.793945 & 4.309599 \\
\hline $\mathrm{Zn}$ & 3.895722 & -5.558235 & 3.343403 \\
\hline $\mathrm{O}$ & 5.550928 & -5.131509 & 2.321283 \\
\hline $\mathrm{O}$ & 2.653026 & -5.622035 & 6.920064 \\
\hline $\mathrm{O}$ & 3.251224 & -7.230669 & 4.071322 \\
\hline $\mathrm{O}$ & 8.730319 & -5.844693 & 3.381484 \\
\hline $\mathrm{H}$ & 4.638969 & -1.218373 & 5.330881 \\
\hline $\mathrm{H}$ & 4.485966 & 0.396303 & 6.022115 \\
\hline $\mathrm{H}$ & 3.773505 & -0.581462 & 8.188961 \\
\hline $\mathrm{H}$ & 3.878866 & -2.211367 & 7.491777 \\
\hline
\end{tabular}




$\begin{array}{lrrr}\mathrm{H} & 5.847929 & -1.657628 & 8.657828 \\ \mathrm{H} & 2.180048 & -1.594453 & 5.680234 \\ \mathrm{H} & 2.030838 & 0.037883 & 6.329904 \\ \mathrm{H} & 2.881213 & 0.958734 & 4.153953 \\ \mathrm{H} & 2.995780 & -0.679479 & 3.520180 \\ \mathrm{H} & 0.531798 & -0.964763 & 3.795910 \\ \mathrm{H} & 0.409231 & 0.667824 & 4.443201 \\ \mathrm{H} & 1.293573 & 1.588658 & 2.292102 \\ \mathrm{H} & 1.421969 & -0.041300 & 1.645513 \\ \mathrm{H} & -1.048753 & -0.329934 & 1.878342 \\ \mathrm{H} & -1.179804 & 1.297750 & 2.528570 \\ \mathrm{H} & -1.738201 & 1.270714 & 0.084172 \\ \mathrm{H} & -0.289661 & 2.239620 & 0.376069 \\ \mathrm{H} & -0.141419 & 0.605077 & -0.277618\end{array}$

